Copper-Catalyzed Regioselective 1, 2-Alkylesterification of Dienes to Allylic Esters Yougui Li, ${ }^{[a]}$ Yulong Han,,${ }^{[a, b]}$ Haigen Xiong, ${ }^{[b]}$ Nengbo Zhu, ${ }^{[b]}$ Bo Qian, ${ }^{[b]}$ Changqing Ye, ${ }^{[b]}$ Eric Assen B. Kantchev ${ }^{[a]}$ and Hongli Bao*[b]

${ }^{[a]}$ School of Chemistry and Chemical Engineering, Hefei University of Technology, 193 Tunxi Road, Hefei, 230009, China

${ }^{[b]}$ Key Laboratory of Coal to Ethylene Glycol and Its Related Technology, Fujian Institute of Research on the Structure of Matter, Chinese Academy of Sciences, 155 Yangqiao Road West, Fuzhou, 350002, P.R. China

Table of Contents:

Materials and Methods

Synthesis of Diacyl Peroxides $\quad$ S-1

Synthesis of 1,3-diene

S-4

General Procedure for the Catalytic 1, 2-Alkylesterification of Butadiene $\quad$ S-4

Characterization Data for Products $\quad$ S-5

Radical Trapping Reaction $\quad$ S-11

Crossover Reaction with Two Effective Alkyl Diacyl Peroxides $\quad$ S-13

Crossover Reaction between Effective and Noneffective Diacyl Peroxides S-14

$\begin{array}{ll}\text { NMR Spectra } & \text { S-15 }\end{array}$

$\begin{array}{ll}\text { References } & \text { S-48 }\end{array}$

Materials and Methods

All reactions were carried out under an atmosphere of nitrogen in flame-dried glassware with magnetic stirring unless otherwise indicated. Commercially obtained reagents were used as received. Solvents were dried by distillation from sodium or $\mathrm{CaH}_{2}$. Liquids and solutions were transferred via syringe. All reactions were monitored by thin-layer chromatography. ${ }^{1} \mathrm{H}$ and ${ }^{13} \mathrm{C}$ NMR spectra were recorded on Bruker-BioSpin AVANCE III HD. Data for ${ }^{1} \mathrm{H}$ NMR spectra are reported relative to chloroform as an internal standard $(7.26 \mathrm{ppm})$ and are reported as follows: chemical shift (ppm), multiplicity, coupling constant $(\mathrm{Hz})$, and integration. Data for ${ }^{13} \mathrm{C}$ NMR spectra are reported relative to chloroform as an internal standard (77.23 ppm) and are reported in terms of chemical shift (ppm). IR data were obtained from Bruker VERTEX 70. HRMS data were recorded on Agilent Technologies 6224 TOF LC/MS.

Synthesis of Diacyl Peroxides ${ }^{1}$<smiles>[R]CC(=O)OOC(=O)C[R]</smiles>

Diacyl peroxides have potentials to explode. Any diacyl peroxide involved reaction (as product or substrate) should be carried out with precautions!

Besides lauroyl peroxide (LPO) 2a was purchased from $J \& K^{\circledR}$ and diacyl peroxide $2 \mathbf{i}$ was purchased from TCI, other diacyl peroxides were prepared by the following general procedure: 
A solution of pyridine $(3.16 \mathrm{~mL}, 40 \mathrm{mmol})$ in ether $(1.0 \mathrm{~mL})$ was cooled to $-10{ }^{\circ} \mathrm{C}$ and $30 \%$ hydrogen peroxide $(1.25 \mathrm{~mL}, 11 \mathrm{mmol})$ was added dropwise. The mixture was rapidly stirred so that the two-phase system was finely dispersed. The acid chloride $(20 \mathrm{mmol})$ was then added dropwise, maintaining the temperature between -5 and $-10^{\circ} \mathrm{C}$. The mixture was then stirred for 2 additional hours at $0{ }^{\circ} \mathrm{C}$ and carefully neutralized with a chilled $10 \%$ sulfuric acid solution. Ether $(20 \mathrm{~mL})$ was added and the peroxide extracted (throughout this and subsequent operations the temperature was not allowed to rise above $0{ }^{\circ} \mathrm{C}$ ). The aqueous layer was extracted further with pentane. The ether and pentane extracts were combined, washed with chilled $10 \%$ sulfuric acid, $10 \%$ aqueous sodium carbonate, and finally with water. The solution was dried over anhydrous sodium sulfate and concentrated on a rotary evaporator then on a vacuum pump. The residue was purified by flash chromatography (hexanes/ethyl acetate $=20 / 1 \sim 15 / 1$ ) to afforded the desired product.<smiles>CC(CCCc1ccccc1)OOC(=O)CCCc1ccccc1</smiles>

2b: Following the general procedure for the synthesis of diacyl peroxides, purification by flash chromatography afforded the product $(2.34 \mathrm{~g}, 72 \%$ yield $)$ as a clear oil. ${ }^{1} \mathrm{H}$ NMR $(400 \mathrm{MHz}$, $\left.\mathrm{CDCl}_{3}\right) \delta 7.34(\mathrm{t}, J=7.52 \mathrm{~Hz}, 4 \mathrm{H}), 7.28-7.23(\mathrm{~m}, 6 \mathrm{H}), 7.26(\mathrm{t}, J=7.44 \mathrm{~Hz}, 4 \mathrm{H}), 2.48(\mathrm{t}, J=$ $7.36 \mathrm{~Hz}, 4 \mathrm{H}), 2.13-2.06(\mathrm{~m}, 4 \mathrm{H}) .{ }^{13} \mathrm{C} \mathrm{NMR}\left(100 \mathrm{MHz}, \mathrm{CDCl}_{3}\right) \delta 169.08,140.77,128.61,128.59$, 126.28, 34.79, 29.29, 26.47. IR (thin film): 3084, 3062, 3026, 2936, 2863, 1807, 1775, 1499, $1456,1181,1088,1070,884,744,698 \mathrm{~cm}^{-1}$.

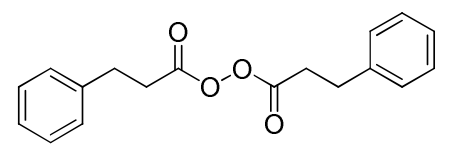

2c: Following the general procedure for the synthesis of diacyl peroxides, purification by flash chromatography afforded the product $(2.05 \mathrm{~g}, 69 \%$ yield $)$ as a clear oil. ${ }^{1} \mathrm{H}$ NMR $(400 \mathrm{MHz}$, $\left.\mathrm{CDCl}_{3}\right) \delta 7.31(\mathrm{t}, J=7.03 \mathrm{~Hz}, 4 \mathrm{H}), 7.25-7.20(\mathrm{~m}, 6 \mathrm{H}), 3.03(\mathrm{t}, J=7.64 \mathrm{~Hz}, 4 \mathrm{H}), 2.74(\mathrm{t}, J=8.20$ $\mathrm{Hz}, 4 \mathrm{H}) .{ }^{13} \mathrm{C}$ NMR $\left(100 \mathrm{MHz}, \mathrm{CDCl}_{3}\right) \delta 168.47,139.38,128.76,128.35,126.76,31.71,30.71$. IR (thin film): 3019, 3091, 3062, 3031, 2931, 2965, 1810, 1783, 1561, 1493, 1457, 1064, 1023, $746,693 \mathrm{~cm}^{-1}$.

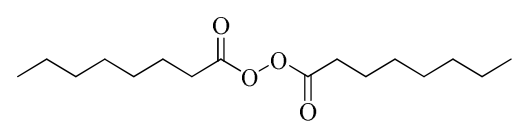

2d: Following the general procedure for the synthesis of diacyl peroxides, purification by flash chromatography afforded the product $(2.75 \mathrm{~g}, 96 \%$ yield $)$ as a clear oil. ${ }^{1} \mathrm{H}$ NMR $(400 \mathrm{MHz}$, $\left.\mathrm{CDCl}_{3}\right) \delta 2.43(\mathrm{t}, J=7.40 \mathrm{~Hz}, 4 \mathrm{H}), 1.73-1.68(\mathrm{~m}, 4 \mathrm{H}), 1.37-1.28(\mathrm{~m}, 16 \mathrm{H}), 0.88(\mathrm{t}, J=6.40 \mathrm{~Hz}$, $6 \mathrm{H}) .{ }^{13} \mathrm{C} \mathrm{NMR}\left(100 \mathrm{MHz}, \mathrm{CDCl}_{3}\right) \delta 169.32,31.56,30.04,28.89,28.77,24.83,22.58,14.06 . \mathrm{IR}$ (thin film): 2957, 2931, 2857, 1810, 1781, 1555, 1457, 1132, 1063, 877, $721 \mathrm{~cm}^{-1}$.<smiles>CCCCCC(=O)OC(=O)CCCCC</smiles>

2e: Following the general procedure for the synthesis of diacyl peroxides, purification by flash chromatography afforded the product $\left(1.19 \mathrm{~g}, 52 \%\right.$ yield) as a clear oil. ${ }^{1} \mathrm{H}$ NMR $(400 \mathrm{MHz}$, $\left.\mathrm{CDCl}_{3}\right) \delta 2.35(\mathrm{t}, J=7.44 \mathrm{~Hz}, 4 \mathrm{H}), 1.69-1.61(\mathrm{~m}, 4 \mathrm{H}), 1.32-1.26(\mathrm{~m}, 8 \mathrm{H}), 0.83(\mathrm{t}, J=7.00 \mathrm{~Hz}$, 
6H). ${ }^{13} \mathrm{C} \mathrm{NMR}\left(100 \mathrm{MHz}, \mathrm{CDCl}_{3}\right) \delta 169.24,31.03,29.95,24.49,22.15,13.77$. IR (thin film): 2956, 2934, 2872, 1815, 1783, 1465, 1121, 1057, 872, $732 \mathrm{~cm}^{-1}$.<smiles>CC(CCCCC(=O)OCc1ccccc1)OOC(=O)CCCCC(=O)OCc1ccccc1</smiles>

2f: Following the general procedure for the synthesis of diacyl peroxides, purification by flash chromatography afforded the product $\left(3.0 \mathrm{~g}, 64 \%\right.$ yield) as a clear oil. ${ }^{1} \mathrm{H}$ NMR $(400 \mathrm{MHz}$, $\left.\mathrm{CDCl}_{3}\right) \delta 7.36-7.32(\mathrm{~m}, 10 \mathrm{H}), 5.11(\mathrm{~s}, 4 \mathrm{H}), 2.46-2.38(\mathrm{~m}, 8 \mathrm{H}), 1.75-1.73(\mathrm{~m}, 8 \mathrm{H}) .{ }^{13} \mathrm{C}$ NMR $(100$ $\left.\mathrm{MHz}, \mathrm{CDCl}_{3}\right) \delta 172.88,168.77,135.93,128.60,128.27,128.26,66.31,33.67,29.66,24.20$, 24.11. IR (thin film): 3090, 3065, 3032, 2943, 2873, 1811, 1780, 1735, 1454, 1416, 1386, 1356, $1251,1217,1161,1090,1052,737,696 \mathrm{~cm}^{-1}$. HRMS (ESI) calcd for $\left[\mathrm{C}_{26} \mathrm{H}_{34} \mathrm{NO}_{8}\right]^{+}\left(\left[\mathrm{M}+\mathrm{NH}_{4}\right]^{+}\right)$: 488.2279, found: 488.2282 .<smiles>CC(C)(C)CC(=O)OOC(=O)CC(C)(C)C</smiles>

2g: Following the general procedure for the synthesis of diacyl peroxides, purification by flash chromatography afforded the product $\left(1.87 \mathrm{~g}, 82 \%\right.$ yield) as a clear oil. ${ }^{1} \mathrm{H}$ NMR $(400 \mathrm{MHz}$, $\left.\mathrm{CDCl}_{3}\right) \delta 2.32(\mathrm{~s}, 4 \mathrm{H}), 1.10(\mathrm{~s}, 18 \mathrm{H}) .{ }^{13} \mathrm{C} \mathrm{NMR}\left(100 \mathrm{MHz}, \mathrm{CDCl}_{3}\right) \delta 167.42,43.63,31.09,29.48$. IR (thin film): 2954, 2907, 2874, 1802, 1780, 1477, 1372, 1317, 1239, 1190, 1076, 1027, 920, $848,630 \mathrm{~cm}^{-1}$.<smiles>C=CCCC(=O)OC(=O)CCC=C</smiles>

2h: Following the general procedure for the synthesis of diacyl peroxides, purification by flash chromatography afforded the product $\left(1.60 \mathrm{~g}, 20 \%\right.$ yield) as a clear oil. ${ }^{1} \mathrm{H}$ NMR $(400 \mathrm{MHz}$, $\left.\mathrm{CDCl}_{3}\right) \delta 5.89-5.79(\mathrm{~m}, 2 \mathrm{H}), 5.14-5.06(\mathrm{~m}, 4 \mathrm{H}), 2.56\left(\mathrm{dd}, J_{l}=1.84 \mathrm{~Hz}, J_{2}=8.52 \mathrm{~Hz}, 4 \mathrm{H}\right), 2.49$ $\left(\mathrm{dd}, J_{l}=6.32 \mathrm{~Hz}, J_{2}=13.48 \mathrm{~Hz}, 4 \mathrm{H}\right) .{ }^{13} \mathrm{C}$ NMR $\left(100 \mathrm{MHz}, \mathrm{CDCl}_{3}\right) \delta 168.51,135.40,116.47$, 29.36, 28.55. IR (thin film): 3083, 2903, 2925, 2858, 1814, 1781, 1642, 1418, 1361, 1062, 999, $923,857 \mathrm{~cm}^{-1}$.<smiles>O=C(OOC(=O)C1CC1)C1CC1</smiles>

2j: Following the general procedure for the synthesis of diacyl peroxides, purification by flash chromatography afforded the product $(0.92 \mathrm{~g}, 54 \%$ yield $)$ as a clear solid. ${ }^{1} \mathrm{H}$ NMR $(400 \mathrm{MHz}$, $\left.\mathrm{CDCl}_{3}\right) \delta 1.76-1.70(\mathrm{~m}, 2 \mathrm{H}), 1.18-1.14(\mathrm{~m}, 4 \mathrm{H}), 1.08-1.03(\mathrm{~m}, 4 \mathrm{H}) .{ }^{13} \mathrm{C} \mathrm{NMR}\left(100 \mathrm{MHz}, \mathrm{CDCl}_{3}\right)$ $\delta 170.96,9.40,9.38$. IR (thin film): 3069, 2964, 1800, 1771, 1597, 1452, 1371, 1230, 1199, 1082, $1010,922,832,706 \mathrm{~cm}^{-1}$.<smiles>CC(C)(C)CC(=O)OOC(=O)c1ccccc1</smiles>

2k: Following the general procedure for the synthesis of diacyl peroxides (40 mmol of acid chloride), purification by flash chromatography afforded the product ( $4.0 \mathrm{~g}, 42 \%$ yield) as a clear 
solid. ${ }^{1} \mathrm{H}$ NMR $\left(400 \mathrm{MHz}, \mathrm{CDCl}_{3}\right) \delta 8.03(\mathrm{~d}, J=8.00 \mathrm{~Hz}, 2 \mathrm{H}), 7.63(\mathrm{t}, J=8.00 \mathrm{~Hz}, 1 \mathrm{H}), 7.48(\mathrm{t}$, $J=8.00 \mathrm{~Hz}, 2 \mathrm{H}), 2.40(\mathrm{~s}, 2 \mathrm{H}), 1.15(\mathrm{~s}, 9 \mathrm{H}) .{ }^{13} \mathrm{C} \mathrm{NMR}\left(100 \mathrm{MHz}, \mathrm{CDCl}_{3}\right) \delta 167.51,163.02$, 134.16, 129.73, 128.80, 125.73, 43.68, 31.21, 29.51. IR (thin film): 3069, 2964, 2869, 1800, $1766,1600,1449,1371,1226,1199,1082,1013,918,832,706 \mathrm{~cm}^{-1}$.

\section{Synthesis of 1,3-Diene}

Most 1,3-Diene substrates were obtained from commercial sources. The 1,3-diene 5d was prepared according to a literature procedure. ${ }^{2}$ The 1,3 -diene $\mathbf{5 f}$ was prepared according to the literature procedure. ${ }^{3}$ The 1,3 -diene $\mathbf{5 g}$ was also prepared according to the known report. ${ }^{4}$<smiles>C=CC(=C)CCc1ccccc1</smiles>

\section{$5 d$}

${ }^{1} \mathrm{H}$ NMR $\left(400 \mathrm{MHz}, \mathrm{CDCl}_{3}\right) \delta 7.29(\mathrm{t}, J=8.00 \mathrm{~Hz}, 2 \mathrm{H}), 7.21-7.19(\mathrm{~m}, 3 \mathrm{H}), 6.44\left(\mathrm{dd}, J_{l}=12.00\right.$ $\left.\mathrm{Hz}, J_{2}=16.00 \mathrm{~Hz}, 1 \mathrm{H}\right), 5.30(\mathrm{~d}, J=16.00 \mathrm{~Hz}, 1 \mathrm{H}), 5.10(\mathrm{~d}, J=8.00 \mathrm{~Hz}, 1 \mathrm{H}), 5.04(\mathrm{~d}, J=16.00$ $\mathrm{Hz}, 2 \mathrm{H}), 2.81(\mathrm{t}, J=8.00 \mathrm{~Hz}, 2 \mathrm{H}), 2.52(\mathrm{t}, J=8.00 \mathrm{~Hz}, 2 \mathrm{H})$.<smiles>C=C(C)C=CCO</smiles>

$5 f$

${ }^{1} \mathrm{H}$ NMR $\left(400 \mathrm{MHz}, \mathrm{CDCl}_{3}\right) \delta 6.37(\mathrm{~d}, J=16.00 \mathrm{~Hz}, 1 \mathrm{H}), 5.84-5.79(\mathrm{~m}, 1 \mathrm{H}), 4.99(\mathrm{~s}, 2 \mathrm{H}), 4.24$ $(\mathrm{d}, J=8.00 \mathrm{~Hz}, 2 \mathrm{H}), 1.86(\mathrm{~s}, 3 \mathrm{H})$.<smiles>C=C(C)/C=C/CO[Si](C)(C)C(C)(C)C</smiles>

${ }^{1} \mathrm{H}$ NMR $\left(400 \mathrm{MHz}, \mathrm{CDCl}_{3}\right) \delta 6.34(\mathrm{~d}, J=16.00 \mathrm{~Hz}, 1 \mathrm{H}), 5.75(\mathrm{~d}, J=16.00 \mathrm{~Hz}, 1 \mathrm{H}), 4.95(\mathrm{~s}$, $2 \mathrm{H}), 4.26(\mathrm{~s}, 2 \mathrm{H}), 1.85(\mathrm{~s}, 3 \mathrm{H}), 0.92(\mathrm{~s}, 9 \mathrm{H}), 0.09(\mathrm{~s}, 6 \mathrm{H})$.

\section{General Procedure for the Catalytic 1,2-Difunctionalizations of 1,3-diene}

General Procedure for Table 1:

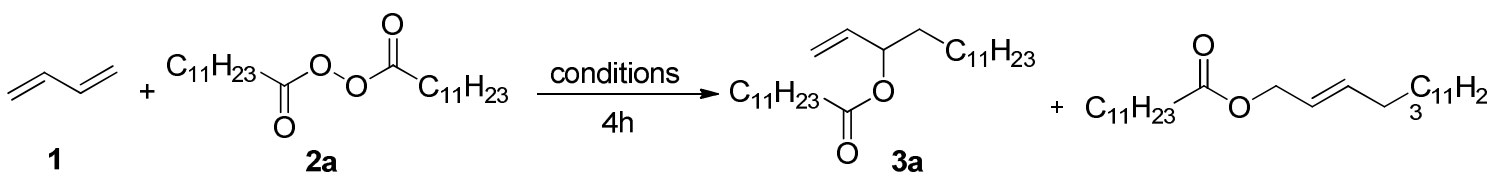

Lauryl peroxide $\mathbf{2 a}$ (398 $\mathrm{mg}, 1 \mathrm{mmol})$, catalyst and 1,4-dioxane $(2 \mathrm{~mL})$ were added into a flame dried schlenk tube with a stirring bar. 1,3-butadiene 1 was bubbled into the reaction solution at $70{ }^{\circ} \mathrm{C}$ for 4 hours. Then, the reaction mixture was cooled to ambient temperature, poured into saturated $\mathrm{NaCl}$ solution $(50 \mathrm{~mL})$ and extracted with $\mathrm{CH}_{2} \mathrm{Cl}_{2}(3 \times 50 \mathrm{~mL})$. The combined organic phase was dried over anhydrous $\mathrm{MgSO}_{4}$. After filtration and evaporation of the solvents under reduced pressure, the residue was purified by column chromatography on silica gel (petroleum ether/ethyl acetate $=100 / 0 \sim 20 / 1)$ to yield 3a.

General Procedure for Table 2: 


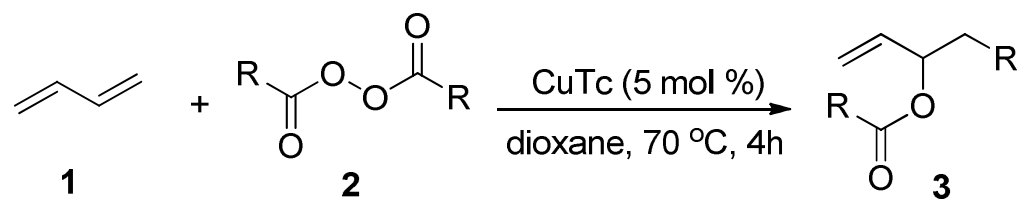

Diacyl peroxide 2 (2 mmol), CuTc (19.4 mg, $0.05 \mathrm{mmol})$ and 1,4-dioxane $(4 \mathrm{~mL})$ were added into a flame dried schlenk tube with a stirring bar. 1,3-butadiene 1 was bubbled into the reaction solution at $70{ }^{\circ} \mathrm{C}$ for 4 hours. Then, the reaction mixture was cooled to ambient temperature, poured into saturated $\mathrm{NaCl}$ solution $(50 \mathrm{~mL})$ and extracted with $\mathrm{CH}_{2} \mathrm{Cl}_{2}(3 \times 50 \mathrm{~mL})$. The combined organic phase was dried over anhydrous $\mathrm{MgSO}_{4}$. After filtration and evaporation of the solvents under reduced pressure, the residue was purified by column chromatography on silica gel (petroleum ether/ethyl acetate $=100 / 0 \sim 20 / 1$ ) to yield $\mathbf{3}$.

General Procedure for Table 3:

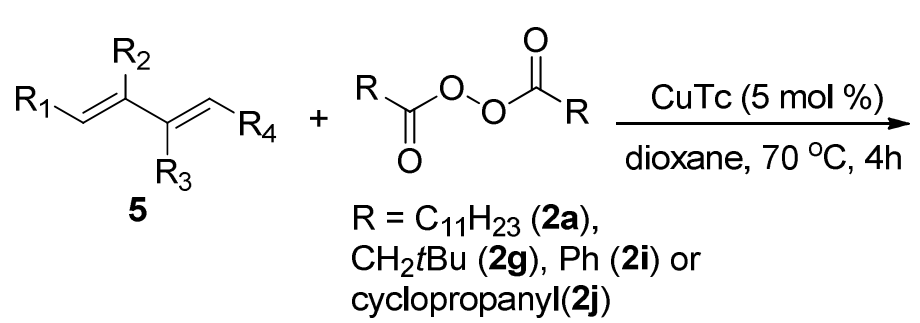<smiles>[R]C=C([R])C([R])(OC([R])=O)C([R])[R]</smiles>

1,3-Diene 5 (1 equiv), diacyl peroxide $\mathbf{2 a}, \mathbf{2 g}, \mathbf{2 i}$, or $\mathbf{2 j}$ (1.75 equiv), CuTc (5 mmol\%) and 1,4dioxane $(2 \mathrm{~mL} / \mathrm{mmol})$ were added into a flame dried schlenk tube with a stirring bar. The mixture was heated to $70{ }^{\circ} \mathrm{C}$ and kept at this temperature for 4 hours. Then, the reaction mixture was cooled to ambient temperature, poured into saturated $\mathrm{NaCl}$ solution $(50 \mathrm{~mL})$ and extracted with $\mathrm{CH}_{2} \mathrm{Cl}_{2}(3 \times 50 \mathrm{~mL})$. The combined organic phase was dried over anhydrous $\mathrm{MgSO}_{4}$. After filtration and evaporation of the solvents under reduced pressure, the crude product was purified by column chromatography on silica gel (petroleum ether/ethyl acetate $=100 / 0 \sim 20 / 1$ ) to yield 616.

\section{Characterization Data for Products.}

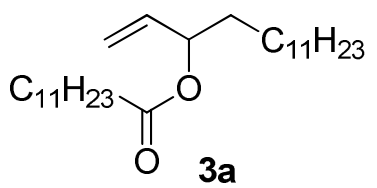

Table 1: Following the general procedure for Table 1, purification by column chromatography afforded the product $(281.5 \mathrm{mg}, 69 \%)$ as a white solid: ${ }^{1} \mathrm{H} \mathrm{NMR}\left(400 \mathrm{MHz}, \mathrm{CDCl}_{3}\right) \delta 5.23$ $5.22(\mathrm{~m}, 1 \mathrm{H}), 5.28-5.25(\mathrm{~m}, 1 \mathrm{H}), 5.23-5.22(\mathrm{~m}, 2 \mathrm{H}), 2.32(\mathrm{t}, J=7.60 \mathrm{~Hz}, 2 \mathrm{H}), 1.66-1.61(\mathrm{~m}, 4 \mathrm{H})$, $1.28(\mathrm{~s}, 36 \mathrm{H}), 0.90(\mathrm{t}, J=5.64 \mathrm{~Hz}, 6 \mathrm{H}) .{ }^{13} \mathrm{C}$ NMR $\left(100 \mathrm{MHz}, \mathrm{CDCl}_{3}\right) \delta 173.16,136.85,116.31$, 74.51, 34.66, 34.25, 31.93, 29.66, 29.62, 29.58, 29.53, 29.49, 29.37, 29.35, 29.29, 29.17, 25.08, 22.69, 14.11. IR (thin film): $3088,2931,2853,1742,1469,1174,1161,1109,987,927,718 \mathrm{~cm}^{-1}$. HRMS (ESI) calcd for $\left[\mathrm{C}_{27} \mathrm{H}_{56} \mathrm{NO}_{2}\right]^{+}\left(\left[\mathrm{M}+\mathrm{NH}_{4}\right]^{+}\right)$: 426.4306 , found: 426.4306 . 
<smiles>C=CC(CCCCc1ccccc1)OC(=O)CCCCc1ccccc1</smiles>

Table 2, entry 1: Following the general procedure for Table 2, purification by column chromatography (washing with petroleum ether and ethyl acetate) afforded the product (483.8 $\mathrm{mg}, 72 \%$ yield) as a white solid: ${ }^{1} \mathrm{H}$ NMR $\left(400 \mathrm{MHz}, \mathrm{CDCl}_{3}\right) \delta 7.30-7.24(\mathrm{~m}, 4 \mathrm{H}), 7.21-7.14(\mathrm{~m}$, $6 \mathrm{H}), 5.27-5.24(\mathrm{~m}, 1 \mathrm{H}), 5.27-5.24(\mathrm{~m}, 1 \mathrm{H}), 5.21-5.13(\mathrm{~m}, 2 \mathrm{H}), 2.66-2.58(\mathrm{~m}, 4 \mathrm{H}), 2.32(\mathrm{t}, J=$ $13.32 \mathrm{~Hz}, 2 \mathrm{H}), 1.96-1-92(\mathrm{~m}, 2 \mathrm{H}), 1.66-1.57(\mathrm{~m}, 4 \mathrm{H}), 1.33(\mathrm{~d}, J=20.4 \mathrm{~Hz}, 2 \mathrm{H}) .{ }^{13} \mathrm{C} \mathrm{NMR}(100$ $\left.\mathrm{MHz}, \mathrm{CDCl}_{3}\right) \delta 172.80,142.42,141.48,136.63,128.54,128.43,128.41,128.31,128.27,126.02$, $125.73,116.70,74.59,35.80,35.18,34.07,33.93,31.20,26.65,24.76$. IR (thin film): 3087, 3063, 3023, 2932, 2855, 1729, 1600, 1488, 1449, 966, 744, $698 \mathrm{~cm}^{-1}$. HRMS (ESI) calcd for $\left[\mathrm{C}_{23} \mathrm{H}_{32} \mathrm{NO}_{2}\right]^{+}\left(\left[\mathrm{M}+\mathrm{NH}_{4}\right]^{+}\right): 354.2428$, found: 354.2429 .<smiles>C=CC(CCCc1ccccc1)OC(=O)CCc1ccccc1</smiles>

Table 2, entry 2: Following the general procedure for Table 2, purification by column chromatography (washing with petroleum ether and ethyl acetate) afforded the product (363.4 $\mathrm{mg}, 59 \%$ yield) as a clear oil: ${ }^{1} \mathrm{H}$ NMR $\left(400 \mathrm{MHz}, \mathrm{CDCl}_{3}\right) \delta$ 7.29-7.24 (m, 4H), 7.20-7.14 (m, $6 \mathrm{H}), 5.76-5.68(\mathrm{~m}, 1 \mathrm{H}), 5.28-5.26(\mathrm{~m}, 1 \mathrm{H}), 5.19-5.11(\mathrm{~m}, 2 \mathrm{H}), 2.95(\mathrm{t}, \mathrm{J}=7.6 \mathrm{~Hz}, 2 \mathrm{H}), 2.65-2.57$ $(\mathrm{m}, 4 \mathrm{H}), 1.62-1.58(\mathrm{~m}, 4 \mathrm{H}) .{ }^{13} \mathrm{C} \mathrm{NMR}\left(100 \mathrm{MHz}, \mathrm{CDCl}_{3}\right) \delta 172.23,142.04,140.50,136.40$, $128.51,128.43,128.37,128.34,126.28,125.87,116.77,74.68,36.13,35.58,33.74,31.01,26.85$. IR (thin film): 3086, 3062, 2936, 2860, 1734, 1496, 1451, 1167, 1077, 967, 928, 751, $696 \mathrm{~cm}^{-1}$. HRMS (ESI) calcd for $\left[\mathrm{C}_{21} \mathrm{H}_{28} \mathrm{NO}_{2}\right]^{+}\left(\left[\mathrm{M}+\mathrm{NH}_{4}\right]^{+}\right)$: 326.2115 , found: 326.2117 .<smiles>C=CC(CC)OC(=O)CCC</smiles>

Table 2, entry 3: Following the general procedure for Table 2, purification by column chromatography (washing with petroleum ether and ethyl acetate) afforded the product (414.4 $\mathrm{mg}, 70 \%$ yield) as a clear oil: ${ }^{1} \mathrm{H}$ NMR $\left(400 \mathrm{MHz}, \mathrm{CDCl}_{3}\right) \delta 5.81-5.73(\mathrm{~m}, 1 \mathrm{H}), 5.26-5.23(\mathrm{~m}$, $1 \mathrm{H}), 5.20-5.13(\mathrm{~m}, 2 \mathrm{H}), 2.31(\mathrm{t}, J=7.36 \mathrm{~Hz}, 2 \mathrm{H}), 1.63-1.58(\mathrm{~m}, 4 \mathrm{H}), 1.29-1.26(\mathrm{~m}, 20 \mathrm{H}), 0.88(\mathrm{t}$, $J=6.48 \mathrm{~Hz}, 6 \mathrm{H}) .{ }^{13} \mathrm{C} \mathrm{NMR}\left(100 \mathrm{MHz}, \mathrm{CDCl}_{3}\right) \delta 173.19,136.81,116.34,74.52,34.64,34.23$, $31.86,31.69,29.48,29.38,29.23,29.11,28.96,25.07,22.67,22.61,14.10,14.06$. IR (thin film): 3093, 2954, 2924, 2853, 1737, 1465, 1373, 1164, 1104, 964, $924 \mathrm{~cm}^{-1}$. HRMS (ESI) calcd for $\left[\mathrm{C}_{19} \mathrm{H}_{40} \mathrm{NO}_{2}\right]^{+}\left(\left[\mathrm{M}+\mathrm{NH}_{4}\right]^{+}\right): 314.3054$, found: 314.3055 .<smiles>C=CC(Cc1ccccc1)OC(=O)c1ccccc1</smiles>

Table 2, entry 4: Following the general procedure for Table 2, purification by column chromatography afforded the product (374.4 mg, 78\% yield) as a clear oil: ${ }^{1} \mathrm{H}$ NMR (400 MHz, 
$\left.\mathrm{CDCl}_{3}\right) \delta 5.82-5.73(\mathrm{~m}, 1 \mathrm{H}), 5.26-5.23(\mathrm{~m}, 1 \mathrm{H}), 5.20-5.13(\mathrm{~m}, 2 \mathrm{H}), 2.31(\mathrm{t}, J=7.48 \mathrm{~Hz}, 2 \mathrm{H})$, $1.67-1.59(\mathrm{~m}, 4 \mathrm{H}), 1.32-1.27(\mathrm{~m}, 12 \mathrm{H}), 0.91-0.86(\mathrm{~m}, 6 \mathrm{H}) .{ }^{13} \mathrm{C}$ NMR $\left(100 \mathrm{MHz}, \mathrm{CDCl}_{3}\right) \delta 173.23$, 136.82, 116.37, 74.54, 34.61, 34.23, 31.72, 31.32, 29.04, 25.04, 24.75, 22.58, 22.35, 14.08, 13.94. IR (thin film): 3090, 2959, 2928, 2857, 1740, 1465, 1381, 1245, 1169, 1097, 987, 967, $927 \mathrm{~cm}^{-1}$. HRMS (ESI) calcd for $\left[\mathrm{C}_{15} \mathrm{H}_{32} \mathrm{NO}_{2}\right]^{+}\left(\left[\mathrm{M}+\mathrm{NH}_{4}\right]^{+}\right): 258.2428$, found: 258.2430 .<smiles>C=CC(CCCCCCC(=O)OCc1ccccc1)OC(=O)CCCCCCCCOCc1ccccc1</smiles>

Table 2, entry 5: Following the general procedure for Table 2, purification by column chromatography afforded the product $\left(449.6 \mathrm{mg}, 51 \%\right.$ yield) as a clear oil: ${ }^{1} \mathrm{H}$ NMR $(400 \mathrm{MHz}$, $\left.\mathrm{CDCl}_{3}\right) \delta 7.35-7.31(\mathrm{~m}, 10 \mathrm{H}), 5.78-5.70(\mathrm{~m}, 1 \mathrm{H}), 5.23-5.22(\mathrm{~m}, 1 \mathrm{H}), 5.20-5.13\left(\mathrm{dd}, J_{l}=6.88, J_{2}\right.$ $=18.68 \mathrm{~Hz}, 2 \mathrm{H}), 5.11(\mathrm{~s}, 4 \mathrm{H}), 2.73\left(\mathrm{dd}, J_{l}=6.2, J_{2}=21.48 \mathrm{~Hz}, 6 \mathrm{H}\right), 1.68-1.59(\mathrm{~m}, 8 \mathrm{H}), 1.31-$ $1.26(\mathrm{~m}, 4 \mathrm{H}) .{ }^{13} \mathrm{C}$ NMR $\left(100 \mathrm{MHz}, \mathrm{CDCl}_{3}\right) \delta 173.50,173.15,172.59,136.52,136.09,136.01$, $128.58,128.22,116.75,74.61,66.22,66.14,34.18,34.15,33.97,33.92,28.83,24.78,24.73$, 24.43, 24.38. IR (thin film): 3090, 3066, 3033, 2938, 2860, 1734, 1496, 1456, 1379, 1162, 977 , $748,693 \mathrm{~cm}^{-1}$. HRMS (ESI) calcd for $\left[\mathrm{C}_{29} \mathrm{H}_{40} \mathrm{NO}_{6}\right]^{+}\left(\left[\mathrm{M}+\mathrm{NH}_{4}\right]^{+}\right): 498.2850$, found: 498.2854 .<smiles>C=CC(CCC(C)(C)C)OC(=O)CC(C)(C)C</smiles>

Table 2, entry 6: Following the general procedure for Table 2, purification by column chromatography afforded the product $\left(393.6 \mathrm{mg}, 82 \%\right.$ yield) as a clear oil ${ }^{1} \mathrm{H}$ NMR $(400 \mathrm{MHz}$, $\left.\mathrm{CDCl}_{3}\right) \delta 5.81-5.72(\mathrm{~m}, 1 \mathrm{H}), 5.26-5.22(\mathrm{~m}, 1 \mathrm{H}), 5.21-5.15(\mathrm{~m}, 2 \mathrm{H}), 2.21(\mathrm{~s}, 2 \mathrm{H}), 1.66-1.51(\mathrm{~m}$, $2 \mathrm{H}), 1.27-1.16(\mathrm{~m}, 2 \mathrm{H}), 1.04(\mathrm{~s}, 9 \mathrm{H}), 0.87(\mathrm{~s}, 9 \mathrm{H}) .{ }^{13} \mathrm{C} \mathrm{NMR}\left(100 \mathrm{MHz}, \mathrm{CDCl}_{3}\right) \delta 171.68$, $136.83,116.76,75.15,48.22,38.99,30.82,30.01,29.70,29.44,29.29$. IR (thin film): 3090,2955 , $2870,1736,1475,1360,1230,1129,984,970 \mathrm{~cm}^{-1}$. HRMS (ESI) calcd for $\left[\mathrm{C}_{15} \mathrm{H}_{28} \mathrm{NaO}_{2}\right]^{+}$ $\left([\mathrm{M}+\mathrm{Na}]^{+}\right): 263.1982$, found: 263.1983 .<smiles>C=CCCCC(C=C)OC(=O)CCCC</smiles>

Table 2, entry 7: Following the general procedure for Table 2, purification by column chromatography afforded the product $\left(316.2 \mathrm{mg}, 76 \%\right.$ yield) as a clear oil: ${ }^{1} \mathrm{H}$ NMR $(400 \mathrm{MHz}$, $\left.\mathrm{CDCl}_{3}\right) \delta$ 5.86-5.73 (m, 3H), 5.28-5.25 (m, $\left.1 \mathrm{H}\right), 5.23-5.15(\mathrm{~m}, 2 \mathrm{H}), 5.08-5.02(\mathrm{~m}, 2 \mathrm{H}), 4.99-4.95$ $(\mathrm{m}, 2 \mathrm{H}), 2.49-2.38(\mathrm{~m}, 4 \mathrm{H}), 2.09-2.04(\mathrm{~m}, 2 \mathrm{H}), 1.67-1.58(\mathrm{~m}, 2 \mathrm{H}), 1.46-1.39(\mathrm{~m}, 2 \mathrm{H}) .{ }^{13} \mathrm{C} \mathrm{NMR}$ $\left(100 \mathrm{MHz}, \mathrm{CDCl}_{3}\right) \delta 172.31,138.29,136.67,136.53,116.64,115.50,114.85,74.58,33.75$, 33.60, 33.39, 28.91, 24.30. IR (thin film): 3084, 2981, 2931, 2856, 1735, 1644, 1415, 1251, 1173, 1094, 985, $914 \mathrm{~cm}^{-1}$. HRMS (ESI) calcd for $\left[\mathrm{C}_{13} \mathrm{H}_{20} \mathrm{NaO}_{2}\right]^{+}\left([\mathrm{M}+\mathrm{Na}]^{+}\right): 231.1356$, found: 231.1356. 


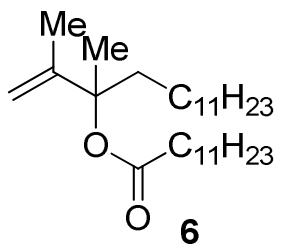

Table 3, entry 1: Following the general procedure for Table $3(3.5 \mathrm{mmol}$ of diacyl peroxide $\mathbf{2 a}$, $2 \mathrm{mmol}$ of 2,3-dimethyl-butadiene $\mathbf{5 a}$, and $4 \mathrm{~mL}$ of 1,4-dioxane), purification by column chromatography afforded the product $(671.4 \mathrm{mg}, 77 \%$ yield $)$ as a clear oil: ${ }^{1} \mathrm{H}$ NMR $(400 \mathrm{MHz}$, $\left.\mathrm{CDCl}_{3}\right) \delta 4.87(\mathrm{~s}, 2 \mathrm{H}), 2.25(\mathrm{t}, J=8.00 \mathrm{~Hz}, 2 \mathrm{H}), 1.84-1.74(\mathrm{~m}, 2 \mathrm{H}), 1.70(\mathrm{~s}, 3 \mathrm{H}), 1.59(\mathrm{t}, J=8.00$ $\mathrm{Hz}, 2 \mathrm{H}), 1.55(\mathrm{~s}, 3 \mathrm{H}), 1.26(\mathrm{~s}, 36 \mathrm{H}), 0.88(\mathrm{t}, J=8.00 \mathrm{~Hz}, 6 \mathrm{H}) .{ }^{13} \mathrm{C}$ NMR $\left(100 \mathrm{MHz}, \mathrm{CDCl}_{3}\right) \delta$ $172.52,147.26,110.69,84.90,38.71,35.30,31.92,29.88,29.68,29.66,29.62,29.59,29.50$, 29.36, 29.34, 29.22, 25.08, 23.65, 23.10, 22.69, 18.96, 14.11. IR (thin film): 3091, 2923, 2853, $1731,1467,1376,1250,1153,1104,892,719 \mathrm{~cm}^{-1}$. HRMS (ESI) calcd for $\left[\mathrm{C}_{29} \mathrm{H}_{56} \mathrm{NaO}_{2}\right]^{+}$ $\left([\mathrm{M}+\mathrm{Na}]^{+}\right): 459.4173$, found: 459.4174 .<smiles>C=C(C)C(C)(CCC(Br)(Br)Br)OC(=O)CBr</smiles>

Table 3, entry 2: Following the general procedure for Table $3(3.5 \mathrm{mmol}$ of diacyl peroxide $\mathbf{2 g}$, $2 \mathrm{mmol}$ of 2,3-dimethyl-butadiene $\mathbf{5 a}$, and $4 \mathrm{~mL}$ of 1,4-dioxane), purification by column chromatography afforded the product $\left(348.4 \mathrm{mg}, 65 \%\right.$ yield) as a clear oil: ${ }^{1} \mathrm{H}$ NMR $(400 \mathrm{MHz}$, $\left.\mathrm{CDCl}_{3}\right) \delta 4.89(\mathrm{~s}, 2 \mathrm{H}), 2.16(\mathrm{~s}, 2 \mathrm{H}), 1.76-1.71(\mathrm{~m}, 5 \mathrm{H}), 1.57(\mathrm{~s}, 3 \mathrm{H}), 1.11-1.04(\mathrm{~m}, 11 \mathrm{H}), 0.87(\mathrm{~s}$, 9H). ${ }^{13} \mathrm{C}$ NMR $\left(100 \mathrm{MHz}, \mathrm{CDCl}_{3}\right) \delta 171.07,146.96,111.01,85.02,48.77,37.31,33.47,30.69$, 29.87, 29.70, 29.33, 22.90, 18.98. IR (thin film): 3071, 2963, 2863, 1800, 1767, 1601, 1449, $1372,1230,1085,1010,918,832,707 \mathrm{~cm}^{-1}$. HRMS (ESI) calcd for $\left[\mathrm{C}_{17} \mathrm{H}_{32} \mathrm{NaO}_{2}\right]^{+}\left([\mathrm{M}+\mathrm{Na}]^{+}\right)$: 291.2295, found: 291.2296 .<smiles>C=C(C)C(Cc1ccccc1)(CC(C)(C)C)c1ccccc1</smiles>

Table 3, entry 3: Following the general procedure for Table 3 ( $3.5 \mathrm{mmol}$ of diacyl peroxide $\mathbf{2 j}$, $2 \mathrm{mmol}$ of 2,3-dimethyl-butadiene $\mathbf{5 a}$, and $4 \mathrm{~mL}$ of 1,4-dioxane), purification by column chromatography afforded the product $(61.6 \mathrm{mg}, 11 \%$ yield $)$, as a clear oil: ${ }^{1} \mathrm{H}$ NMR $(400 \mathrm{MHz}$, $\left.\mathrm{CDCl}_{3}\right) \delta 8.01(\mathrm{~d}, J=8.00 \mathrm{~Hz}, 2 \mathrm{H}), 7.54(\mathrm{t}, J=8.00 \mathrm{~Hz}, 1 \mathrm{H}), 7.42(\mathrm{t}, J=8.00 \mathrm{~Hz}, 2 \mathrm{H}), 7.29$ $7.21(\mathrm{~m}, 5 \mathrm{H}), 4.98(\mathrm{~d}, J=36.00 \mathrm{~Hz}, 2 \mathrm{H}), 3.38(\mathrm{~d}, J=16.00 \mathrm{~Hz}, 1 \mathrm{H}), 3.08(\mathrm{~d}, J=16.00 \mathrm{~Hz}, 1 \mathrm{H})$, $1.81(\mathrm{~s}, 3 \mathrm{H}), 1.68(\mathrm{~s}, 3 \mathrm{H}) .{ }^{13} \mathrm{C}$ NMR $\left(100 \mathrm{MHz}, \mathrm{CDCl}_{3}\right) \delta 164.35,145.94,135.52,131.64$, 129.82, 128.46, 126.82, 125.58, 110.69, 84.33, 44.77, 22.07, 18.33. IR (thin film): 3084,2932 , $2862,1720,1451,1279,1172,1108,1027,899.712 \mathrm{~cm}^{-1}$. HRMS (ESI) calcd for $\left[\mathrm{C}_{19} \mathrm{H}_{24} \mathrm{NO}_{2}\right]^{+}$ $\left(\left[\mathrm{M}+\mathrm{NH}_{4}\right]^{+}\right): 298.1802$, found: 298.1804 . 
<smiles>C=C(C)C(CC1CC1)(CC1CC1)OC(=O)C1CC1</smiles>

Table 3, entry 4: Following the general procedure for Table 2 (1.75 mmol of diacyl peroxide $\mathbf{2 j}$, $1 \mathrm{mmol}$ of 2,3-dimethyl-butadiene $\mathbf{5 a}$, and $2 \mathrm{~mL}$ of 1,4-dioxane), purification by column chromatography afforded the product $\left(81.1 \mathrm{mg}, 39 \%\right.$ yield) as a clear oil: ${ }^{1} \mathrm{H}$ NMR $(400 \mathrm{MHz}$, $\left.\mathrm{CDCl}_{3}\right) \delta 4.90(\mathrm{~d}, J=4.00,2 \mathrm{H}), 1.80\left(\mathrm{dd}, J_{l}=8.00 \mathrm{~Hz}, J_{2}=16.00 \mathrm{~Hz}, 1 \mathrm{H}\right), 1.74(\mathrm{~s}, 3 \mathrm{H}), 1.68(\mathrm{~d}$, $J=4.00 \mathrm{~Hz}, 1 \mathrm{H}), 1.64(\mathrm{~s}, 3 \mathrm{H}), 1.60-1.54(\mathrm{~m}, 1 \mathrm{H}), 0.97-0.93(\mathrm{~m}, 2 \mathrm{H}), 0.81-0.77(\mathrm{~m}, 2 \mathrm{H}), 0.69-$ $0.65(\mathrm{~m}, 1 \mathrm{H}), 0.46-0.44(\mathrm{~m}, 2 \mathrm{H}), 0.06-0.10(\mathrm{~m}, 2 \mathrm{H}) .{ }^{13} \mathrm{C} \mathrm{NMR}\left(100 \mathrm{MHz}, \mathrm{CDCl}_{3}\right) \delta 173.45$, $147.72,110.60,85.28,43.61,23.37,18.95,13.65,7.94,7.81,6.01,4.41,4.24$. IR (thin film): 3069, 2963, 1800, 1771, 1600, 1449, 1371, 1230, 1200, 1082, 1010, 918, 832, $708 \mathrm{~cm}^{-1}$. HRMS (ESI) calcd for $\left[\mathrm{C}_{13} \mathrm{H}_{20} \mathrm{NaO}_{2}\right]^{+}\left([\mathrm{M}+\mathrm{Na}]^{+}\right): 231.1356$, found: 231.1357 .

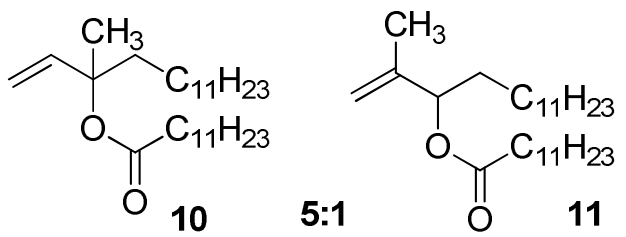

Table 3, entry 5: Following the general procedure for Table 3 (3.5 mmol of diacyl peroxide 2a, $2 \mathrm{mmol}$ of 2,3-dimethyl-butadiene $\mathbf{5 b}$, and $4 \mathrm{~mL}$ of 1,4-dioxane), purification by column chromatography afforded the product $(506.4 \mathrm{mg}, 60 \%$ yield, the ratio of isomers was determined by the ${ }^{1} \mathrm{H}$ NMR) as a clear oil: ${ }^{1} \mathrm{H}$ NMR $\left(400 \mathrm{MHz}, \mathrm{CDCl}_{3}\right) \delta$ 6.01-5.94 (m, 1H), 5.20-5.09 (m, $2 \mathrm{H}), 4.95-4.88(\mathrm{~m}, 1 \mathrm{H}), 2.32(\mathrm{t}, J=7.4 \mathrm{~Hz}, 1 \mathrm{H}), 2.26(\mathrm{t}, J=15.0 \mathrm{~Hz}, 2 \mathrm{H}), 1.73(\mathrm{~s}, 2 \mathrm{H}), 1.68-$ $1.59(\mathrm{~m}, 5 \mathrm{H}), 1.53(\mathrm{~s}, 3 \mathrm{H}), 1.28(\mathrm{~s}, 56 \mathrm{H}), 0.90(\mathrm{t}, J=6.6 \mathrm{~Hz}, 10 \mathrm{H}) .{ }^{13} \mathrm{C} \mathrm{NMR}\left(100 \mathrm{MHz}, \mathrm{CDCl}_{3}\right)$ $\delta 173.12,172.70,143.47,142.21,112.80,112.43,82.82,77.08,39.86,35.41,34.65,32.69,31.94$, $29.63,28.51,29.37,29.36,29.17,25.37,25.11,23.65,23.58,22.70,18.14,14.11$. IR (thin film): 3087, 2929, 2852, 1737, 1470, 1377, 1247, 1162, 1117, 998, 917, $717 \mathrm{~cm}^{-1}$. HRMS (ESI) calcd for $\left[\mathrm{C}_{28} \mathrm{H}_{58} \mathrm{NO}_{2}\right]^{+}\left(\left[\mathrm{M}+\mathrm{NH}_{4}\right]^{+}\right): 440.4462$, found: 440.4464 .

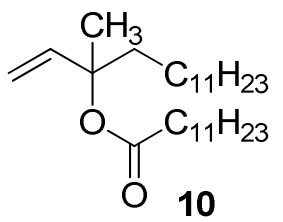

Table 3, entry 5: Following the general procedure for Table 3, purification by column chromatography afforded the product as a clear oil: ${ }^{1} \mathrm{H}$ NMR $\left(400 \mathrm{MHz}, \mathrm{CDCl}_{3}\right) \delta 6.01-5.94(\mathrm{~m}$, $1 \mathrm{H}), 5.17-5.10(\mathrm{~m}, 2 \mathrm{H}), 2.26(\mathrm{t}, J=7.44 \mathrm{~Hz}, 2 \mathrm{H}), 1.66-1.59(\mathrm{~m}, 2 \mathrm{H}), 1.53(\mathrm{~s}, 3 \mathrm{H}), 1.28(\mathrm{~s}, 38 \mathrm{H})$, $0.90(\mathrm{t}, J=6.56 \mathrm{~Hz}, 6 \mathrm{H}) .{ }^{13} \mathrm{C}$ NMR $\left(100 \mathrm{MHz}, \mathrm{CDCl}_{3}\right) \delta 172.74,142.21,112.81,82.83,39.86$, $35.41,31.92$, 29.86, 29.68, 29.66, 29.62, 29.57, 29.50, 29.34, 29.15, 25.10, 23.64, 23.56, 22.69, $22.69,14.10$. 


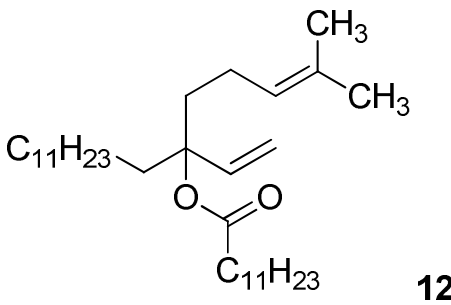

Table 3, entry 6: Following the general procedure for Table 3 (3.5 mmol of diacyl peroxide 2a, $2 \mathrm{mmol}$ of 2,3-dimethyl-butadiene $\mathbf{5 c}$, and $4 \mathrm{~mL}$ of 1,4-dioxane), purification by column chromatography afforded the product $\left(695.8 \mathrm{mg}, 71 \%\right.$ yield) as a clear oil: ${ }^{1} \mathrm{H}$ NMR $(400 \mathrm{MHz}$, $\left.\mathrm{CDCl}_{3}\right) \delta 5.8\left(\mathrm{~d}, J_{l}=11.04 \mathrm{~Hz}, J_{2}=17.48 \mathrm{~Hz}, 1 \mathrm{H}\right), 5.19-5.14(\mathrm{~m}, 2 \mathrm{H}), 5.08(\mathrm{t}, J=7.08 \mathrm{~Hz}, 1 \mathrm{H})$, $2.26(\mathrm{t}, J=7.44 \mathrm{~Hz}, 2 \mathrm{H}), 2.09-2.03(\mathrm{~m}, 2 \mathrm{H}), 1.94-1.86(\mathrm{~m}, 2 \mathrm{H}), 1.70-1.68(\mathrm{~m}, 2 \mathrm{H}), 1.66(\mathrm{~s}, 3 \mathrm{H})$, $1.60(\mathrm{t}, J=7.08 \mathrm{~Hz}, 2 \mathrm{H}), 1.57(\mathrm{~s}, 3 \mathrm{H}), 1.26-1.25(\mathrm{~m}, 36 \mathrm{H}), 0.88(\mathrm{t}, J=6.52 \mathrm{~Hz}, 6 \mathrm{H}) .{ }^{13} \mathrm{C} \mathrm{NMR}$ $\left(100 \mathrm{MHz}, \mathrm{CDCl}_{3}\right) \delta 172.44,141.05,131.61,123.97,113.57,36.55,36.49,35.37,31.93,29.84$, $29.70,29.66,29.63,29.59,29.52,29.35,29.25,25.65,25.18,23.23,22.70,22.06,17.56,14.10$. IR (thin film): 3092, 2960, 2924, 2855, 1741, 1463, 1373, 1166, 1106, 993, 923, $717 \mathrm{~cm}^{-1}$. HRMS (ESI) calcd for $\left[\mathrm{C}_{33} \mathrm{H}_{66} \mathrm{NO}_{2}\right]^{+}\left(\left[\mathrm{M}+\mathrm{NH}_{4}\right]^{+}\right)$: 508.5088 , found: 508.5089 .<smiles>C=CC(C)(CCCBr)OC(=O)CCc1ccccc1</smiles>

Table 3, entry 7: Following the general procedure for Table 3 (3.5 mmol of diacyl peroxide $\mathbf{2 g}$, 2 mmol of 2,3-dimethyl-butadiene $\mathbf{5 d}$, and $4 \mathrm{~mL}$ of 1,4-dioxane), purification by column chromatography afforded the product $\left(357.8 \mathrm{mg}, 52 \%\right.$ yield) as a clear oil: ${ }^{1} \mathrm{H}$ NMR $(400 \mathrm{MHz}$, $\left.\mathrm{CDCl}_{3}\right) \delta 7.29-7.25(\mathrm{~m}, 2 \mathrm{H}), 7.19-7.15(\mathrm{~m}, 3 \mathrm{H}), 5.87\left(\mathrm{dd}, J_{1}=11.04 \mathrm{~Hz}, J_{2}=17.04 \mathrm{~Hz}, 1 \mathrm{H}\right)$, 5.25-5.21 (m, 2H), 2.62-2.50 (m, 2H), 2.45-2.37 (m, 1H), 2.19 (s, 2H), 2.14-1.98 (m, 2H), 1.82$1.74(\mathrm{~m}, 1 \mathrm{H}), 1.18-1.12(\mathrm{~m}, 2 \mathrm{H}), 1.06(\mathrm{~s}, 9 \mathrm{H}), 0.87(\mathrm{~s}, 9 \mathrm{H}) .{ }^{13} \mathrm{C}$ NMR $\left(100 \mathrm{MHz}, \mathrm{CDCl}_{3}\right) \delta$ $171.05,142.15,140.91,128.39,125.80,114.15,85.52$, 48.86, 38.50, 36.94, 31.41, 30.69, 29.98, 29.89, 29.75, 29.71, 29.33. IR (thin film): 3046, 2955, 2920, 2853, 1606, 1506, 1468, 1232, 1158, 1011, 829, $717 \mathrm{~cm}^{-1}$. HRMS (ESI) calcd for $\left[\mathrm{C}_{23} \mathrm{H}_{40} \mathrm{NO}_{2}\right]^{+}\left(\left[\mathrm{M}+\mathrm{NH}^{4}\right]^{+}\right): 362.3054$, found: 362.3057 .

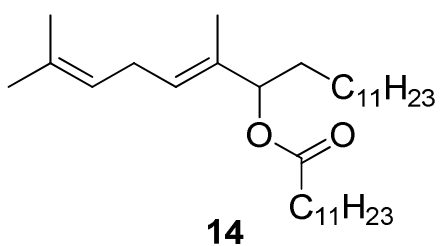

Table 3, entry 8: Following the general procedure for Table 3 ( $1 \mathrm{mmol}$ of 1,3-diene 5e, 1.75 mmol of diacyl peroxide $\mathbf{2 a}$ and $2 \mathrm{~mL}$ of 1,4-dioxane), purification by column chromatography afforded the product $\left(210.7 \mathrm{mg}, 43 \%\right.$ yield) as a clear oil: ${ }^{1} \mathrm{H}$ NMR $\left(400 \mathrm{MHz}, \mathrm{CDCl}_{3}\right) \delta 5.42(\mathrm{t}$, $J=4.00 \mathrm{~Hz}, 1 \mathrm{H}), 5.12(\mathrm{t}, J=4.00 \mathrm{~Hz}, 1 \mathrm{H}), 5.01(\mathrm{t}, J=4.00 \mathrm{~Hz}, 1 \mathrm{H}), 2.29(\mathrm{t}, J=8.00 \mathrm{~Hz}, 2 \mathrm{H})$, $2.02\left(\mathrm{dd}, J_{1}=8.00 \mathrm{~Hz}, J_{2}=12.00 \mathrm{~Hz}, 2 \mathrm{H}\right), 1.67(\mathrm{~s}, 3 \mathrm{H}), 1.61-1.59(\mathrm{~m}, 8 \mathrm{H}), 1.26(\mathrm{~s}, 38 \mathrm{H}), 0.90(\mathrm{t}$, $J=8.00 \mathrm{~Hz}, 6 \mathrm{H}) .{ }^{13} \mathrm{C}$ NMR $\left(100 \mathrm{MHz}, \mathrm{CDCl}_{3}\right) \delta 173.12,133.79,132.61,128.79,119.49,78.89$, $34.75,31.93,31.92,31.88,29.70,29.66,29.63,29.57,29.51,29.37,29.35,29.24,29.15,27.50$, 
25.75, 25.12, 22.70, 17.92, 14.11, 12.00. IR (thin film): 2922, 2856, 1733, 1461, 1377, 1265, $1171,1115,964,803,718 \mathrm{~cm}^{-1}$.

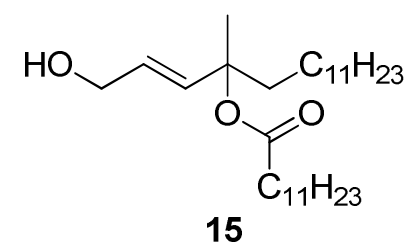

Table 3, entry 9: Following the general procedure for Table 3 ( $1 \mathrm{mmol}$ of 1,3-diene 5f, 1.75 mmol of diacyl peroxide $\mathbf{2 a}$ and $2 \mathrm{~mL}$ of 1,4-dioxane), purification by column chromatography afforded the product $\left(266.7 \mathrm{mg}, 59 \%\right.$ yield) as a clear oil: ${ }^{1} \mathrm{H}$ NMR $\left(400 \mathrm{MHz}, \mathrm{CDCl}_{3}\right) \delta 5.86(\mathrm{~d}$, $J=16.00 \mathrm{~Hz}, 1 \mathrm{H}), 5.76-5.69(\mathrm{~m}, 1 \mathrm{H}), 4.15(\mathrm{~d}, J=4.00 \mathrm{~Hz}, 2 \mathrm{H}), 2.23(\mathrm{t}, J=8.00 \mathrm{~Hz}, 2 \mathrm{H}), 1.80-$ $1.71(\mathrm{~m}, 2 \mathrm{H}), 1.58(\mathrm{t}, J=8.00 \mathrm{~Hz}, 2 \mathrm{H}), 1.51(\mathrm{~s}, 3 \mathrm{H}), 1.26(\mathrm{~s}, 36 \mathrm{H}), 0.88(\mathrm{t}, J=8.00 \mathrm{~Hz}, 6 \mathrm{H}) .{ }^{13} \mathrm{C}$ NMR $\left(100 \mathrm{MHz}, \mathrm{CDCl}_{3}\right) \delta 172.97,135.58,127.74,82.39,63.15,40.19,35.42,31.92,29.68$, $29.66,29.63,29.58,29.51,29.45,29.35,29.31,29.15,25.08,23.94,23.60,22.68,14.10$. IR (thin film): 3086, 2929, 2854, 1719, 1452, 1314, 1277, 1169, 1112, 1088, 1027, 899, $710 \mathrm{~cm}^{-1}$. HRMS (ESI) calcd for $\left[\mathrm{C}_{29} \mathrm{H}_{60} \mathrm{NO}_{3}\right]^{+}\left(\left[\mathrm{M}+\mathrm{NH}_{4}\right]^{+}\right)$: 470.4568, found: 470.4571 .<smiles>CCCCCCCCCCCCCCCCCCCCO[Si](C)(C)C(C)(C)C</smiles>

Table 3, entry 10: Following the general procedure for Table 3 (1 mmol of 1,3-diene 5g, 1.75 mmol of diacyl peroxide $2 \mathbf{a}$ and $2 \mathrm{~mL}$ of 1,4-dioxane), purification by column chromatography afforded the product $\left(238.1 \mathrm{mg}, 42 \%\right.$ yield) as a clear oil: ${ }^{1} \mathrm{H} \mathrm{NMR}\left(400 \mathrm{MHz}, \mathrm{CDCl}_{3}\right) \delta 5.82(\mathrm{~d}$, $J=16.00 \mathrm{~Hz}, 1 \mathrm{H}), 5.69-5.64(\mathrm{~m}, 1 \mathrm{H}), 4.20(\mathrm{~d}, J=4.00 \mathrm{~Hz}, 2 \mathrm{H}), 2.22(\mathrm{t}, J=8.00 \mathrm{~Hz}, 2 \mathrm{H}), 1.88-$ $1.73(\mathrm{~m}, 2 \mathrm{H}), 1.58(\mathrm{t}, J=8.00 \mathrm{~Hz}, 2 \mathrm{H}), 1.51(\mathrm{~s}, 3 \mathrm{H}), 1.25(\mathrm{~s}, 36 \mathrm{H}), 0.91(\mathrm{~s}, 9 \mathrm{H}), 0.88(\mathrm{~s}, 6 \mathrm{H})$, 0.07 (s, 6H). ${ }^{13} \mathrm{C} \mathrm{NMR}\left(100 \mathrm{MHz}, \mathrm{CDCl}_{3}\right) \delta: 172.63,133.94,128.07,82.48,63.51,39.91,35.47$, $31.93,29.69,29.66,29.63,29.60,29.51,29.37,29.35,29.16,25.94,25.82,25.14,24.12,23.68$, 22.69, 18.40, 14.11. IR (thin film): 3086, 2927, 2853, 1719, 1449, 1314, 1276, 1168, 1111, 1087, 1027, 895, $708 \mathrm{~cm}^{-1}$. HRMS (ESI) calcd for $\left[\mathrm{C}_{35} \mathrm{H}_{74} \mathrm{NO}_{3} \mathrm{Si}\right]^{+}\left(\left[\mathrm{M}+\mathrm{NH}_{4}\right]^{+}\right)$: 584.5432 , found: 584.5434 .

\section{Radical Trapping Reaction}

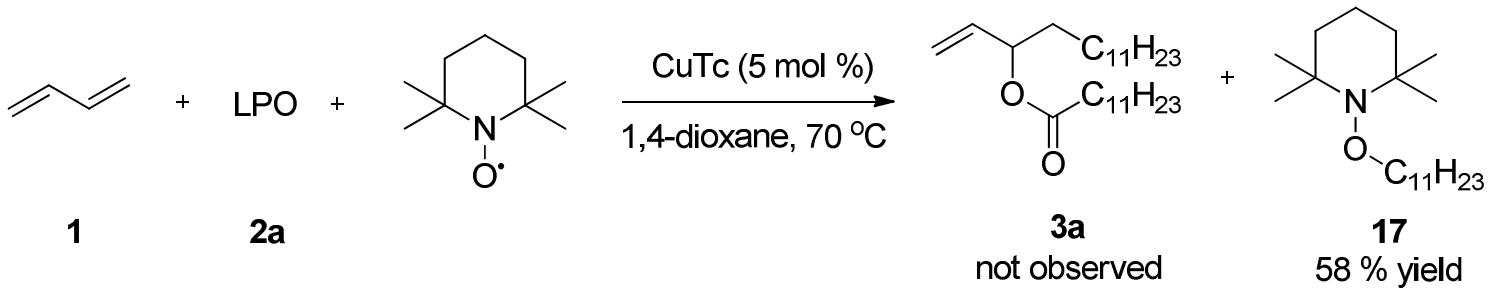

Lauryl peroxide 2a (398 mg, $1 \mathrm{mmol})$, CuTc (9.7 mg, $0.05 \mathrm{mmol})$, TEMPO (156 mg, $1 \mathrm{mmol})$ and 1,4-dioxane $(2 \mathrm{~mL})$ were added into a flame dried schlenk tube with a stirring bar and 1,3butadiene 1 was bubbled into the reaction solution at $70{ }^{\circ} \mathrm{C}$ for 4 hours. Then, the reaction mixture was cooled to ambient temperature, poured into saturated $\mathrm{NaCl}$ solution $(50 \mathrm{~mL})$ and extracted with $\mathrm{CH}_{2} \mathrm{Cl}_{2}(3 \times 50 \mathrm{~mL})$. The combined organic phase was dried over $\mathrm{MgSO}_{4}$. After 
filtration and evaporation of the solvents under reduced pressure, the crude product was purified by column chromatography on silica gel (petroleum ether/ethyl acetate $=100 / 0 \sim 20 / 1$ ) to yield 17 (180.4 mg, 58\%) and 3a was not observed.<smiles>CCCCCCCCCCC1(C)CCCCC1(C)C</smiles>

17

${ }^{1} \mathrm{H}$ NMR $\left(400 \mathrm{MHz}, \mathrm{CDCl}_{3}\right) \delta 3.71(\mathrm{t}, J=8.00 \mathrm{~Hz}, 2 \mathrm{H}), 1.61-1.26(\mathrm{~m}, 24 \mathrm{H}), 1.15(\mathrm{~d}, J=24.00$ $\mathrm{Hz}, 12 \mathrm{H}), 0.88(\mathrm{t}, J=8.00 \mathrm{~Hz}, 3 \mathrm{H}) .{ }^{13} \mathrm{C} \mathrm{NMR}\left(100 \mathrm{MHz}, \mathrm{CDCl}_{3}\right) \delta 76.90,59.58,39.62,33.06$, $31.95,29.76,29.67,29.66,29.64,29.37,28.75,26.48,22.71,20.10,17.17,14.12$.

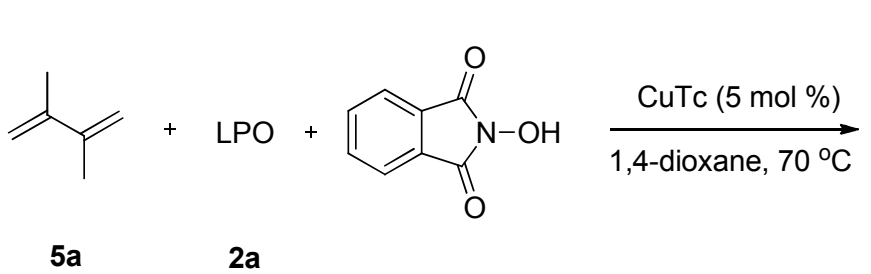

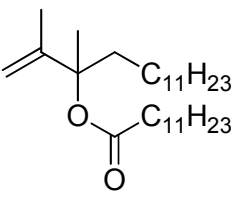

6 $19 \%$ yield

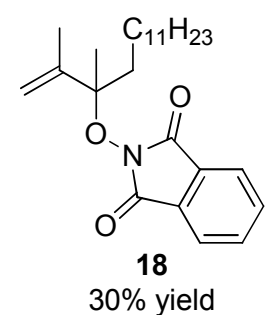

Lauryl peroxide 2a (398 mg, $1 \mathrm{mmol}$ ), 2-hydroxyisoindoline-1,3-dione (164 mg, $1 \mathrm{mmol}), 1,3$ diene $5 \mathbf{a}(123 \mathrm{mg}, 1.5 \mathrm{mmol}), \mathrm{CuTc}(9.7 \mathrm{mg}, 0.05 \mathrm{mmol})$ and 1,4-dioxane $(2 \mathrm{~mL})$ were added into a flame dried schlenk tube with a stirring bar and 1,3-butadiene was bubbled into the reaction solution at $70{ }^{\circ} \mathrm{C}$ for 4 hours. Then, the reaction mixture was cooled to ambient temperature, ured into saturated $\mathrm{NaCl}$ solution $(50 \mathrm{~mL})$ and extracted with $\mathrm{CH}_{2} \mathrm{Cl}_{2}(3 \times 50 \mathrm{~mL})$. The combined organic phase was dried over $\mathrm{MgSO}_{4}$. After filtration and evaporation of the solvents under reduced pressure, the crude product was purified by column chromatography on silica gel (petroleum ether/ethyl acetate $=100 / 0 \sim 20 / 1)$ to yield $\mathbf{6}(82.8 \mathrm{mg}, 19 \%)$ and $\mathbf{1 8}(119.7$ $\mathrm{mg}, 30 \%)$.

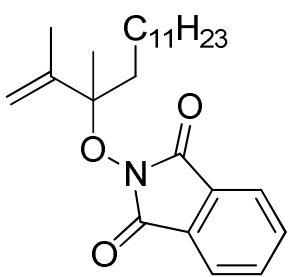

18

${ }^{1} \mathrm{H}$ NMR $\left(400 \mathrm{MHz}, \mathrm{CDCl}_{3}\right) \delta$ 7.82-7.80 (m, 2H), 7.74-7.72 (m, 2H), $5.03(\mathrm{~d}, J=12.00 \mathrm{~Hz}, 2 \mathrm{H})$, $2.02(\mathrm{~s}, 3 \mathrm{H}), 1.98-1.91(\mathrm{~m}, 1 \mathrm{H}), 1.79-1.72(\mathrm{~m}, 1 \mathrm{H}), 1.47(\mathrm{~s}, 3 \mathrm{H}), 1.30-1.26(\mathrm{~m}, 20 \mathrm{H}), 0.88(\mathrm{t}, J=$ $8.00 \mathrm{~Hz}, 3 \mathrm{H}) .{ }^{13} \mathrm{C}$ NMR $\left(100 \mathrm{MHz}, \mathrm{CDCl}_{3}\right) \delta 165.48,145.36,134.31,129.28,123.34,114.9$, $91.85,37.02$, 31.93, 30.10, 29.67, 29.65, 29.62, 29.56, 29.35, 24.75, 22.70, 21.21, 19.33, 14.12. IR (thin film): $3092,2923,2850,1796,1735,1471,1375,1186,1078,965,877,705,520 \mathrm{~cm}^{-1}$. HRMS (ESI) calcd for $\left[\mathrm{C}_{25} \mathrm{H}_{41} \mathrm{~N}_{2} \mathrm{O}_{3}\right]^{+}\left(\left[\mathrm{M}+\mathrm{NH}_{4}\right]^{+}\right)$: 417.3112 , found: 417.3114.

\section{Crossover Reaction between Two Effective Diacyl Peroxides}




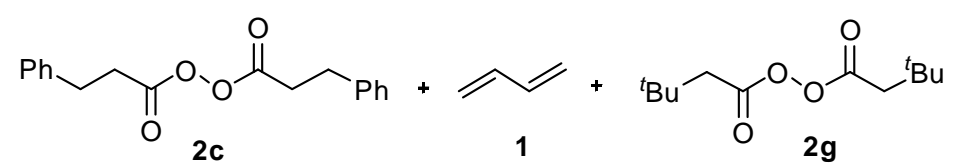<smiles>C=CC(CCCC(C)(C)C)OC(=O)CCC(C=C)OC(=O)CC[Pb]c1ccccc1</smiles>

Diacyl peroxide $2 \mathbf{c}$ (298 mg, $1 \mathrm{mmol}$ ), diacyl peroxide $2 \mathrm{~g}$ (228 mg, $1 \mathrm{mmol})$, CuTc (9.7 mg, 0.05 $\mathrm{mmol})$ and 1,4-dioxane $(2 \mathrm{~mL})$ were added into a flame dried schlenk tube with a stirring bar and 1,3-butadiene 1 was bubbled into the reaction solution at $70{ }^{\circ} \mathrm{C}$ for 4 hours. Then, the reaction mixture was cooled to ambient temperature, poured into saturated $\mathrm{NaCl}$ solution $(50 \mathrm{~mL})$ and extracted with $\mathrm{CH}_{2} \mathrm{Cl}_{2}(3 \times 50 \mathrm{~mL})$. The combined organic phase was dried over $\mathrm{MgSO}_{4}$. After filtration and evaporation of the solvents under reduced pressure, the crude product was purified by column chromatography on silica gel (petroleum ether/ethyl acetate $=100 / 0 \sim 20 / 1$ ) to yield $45 \%$ of the mixed product $\mathbf{3 g}, \mathbf{2 0}, \mathbf{3 c}$ and $\mathbf{2 1}(\mathbf{3 g}: \mathbf{1 9}: \mathbf{3 c}: \mathbf{2 0}=15: 40: 35: 10)$.<smiles>C=CC(CCC(C)(C)C)OC(=O)CCc1ccccc1</smiles>

19<smiles>C=CC(CCCc1ccccc1)OC(=O)CBr</smiles>

20

${ }^{1} \mathrm{H}$ NMR (400 MHz, $\left.\mathrm{CDCl}_{3}\right) \delta$ 7.30-7.27 (m, 2.7H), 7.22-7.15 (m, 3.9H), 5.77-5.70 (m, 1.3H), 5.26-5.13 (m, 3.8H), $2.96(\mathrm{t}, J=8.00 \mathrm{~Hz}, 2.0 \mathrm{H}), 2.65(\mathrm{t}, J=8.00 \mathrm{~Hz}, 2.7 \mathrm{H}), 2.20(\mathrm{~s}, 0.7 \mathrm{H}), 1.72-$ $1.62(\mathrm{~m}, 1.3 \mathrm{H}), 1.57-1.51(\mathrm{~m}, 2.1 \mathrm{H}), 1.18-1.1(\mathrm{~m}, 2.0 \mathrm{H}), 1.03(\mathrm{~s}, 3.1 \mathrm{H}), 0.85(\mathrm{~s}, 9 \mathrm{H})$. IR (thin film): $3086,2927,2853,1719,1452,1309,1280,1169,1111,1088,1027,895,708 \mathrm{~cm}^{-1}$. HRMS (ESI) calcd for $\left[\mathrm{C}_{18} \mathrm{H}_{30} \mathrm{NO}_{2}\right]^{+}\left(\left[\mathrm{M}+\mathrm{NH}_{4}\right]^{+}\right): 292.2271$, found: 292.2273 .

\section{Crossover Reaction between Effective and Noneffective Diacyl Peroxides}<smiles>C=C(C)C(=C)[C+]=C(CC(C)(C)C)c1ccccc1</smiles><smiles>C=C(C)C(C)(CCC(C)(C)C)OC(=O)CC(C)(C)C</smiles><smiles>[Z10]OC(C)(CCC(C)(C)C)C(=C)C</smiles>

$7: 10$

Diacyl peroxide $2 \mathbf{i}$ (242 mg, $1.0 \mathrm{mmol})$, diacyl peroxide $\mathbf{2 g}$ (230 mg, $1.0 \mathrm{mmol})$, CuTc (19.1 mg, $0.1 \mathrm{mmol}), 1,3$-diene $5 \mathbf{a}(246 \mathrm{mg}, 3 \mathrm{mmol})$ and 1,4-dioxane (4 mL) were added into a flame dried schlenk tube with a stirring bar at $70{ }^{\circ} \mathrm{C}$ for 4 hours. Then, the reaction mixture was cooled to ambient temperature, poured into $\mathrm{H}_{2} \mathrm{SO}_{4}$ aqueous solution $(50 \mathrm{~mL})$ and extracted with $\mathrm{CH}_{2} \mathrm{Cl}_{2}$ $(3 \times 50 \mathrm{~mL})$. The combined organic phase was dried over $\mathrm{MgSO}_{4}$. After filtration and evaporation of the solvents under reduced pressure, the crude product was purified by column chromatography on silica gel (petroleum ether/ethyl acetate $=100 / 0 \sim 20 / 1$ ) to yield $35 \%$ of the mixed product 7 and $22(7: \mathbf{2 1}=7: 10)$. 


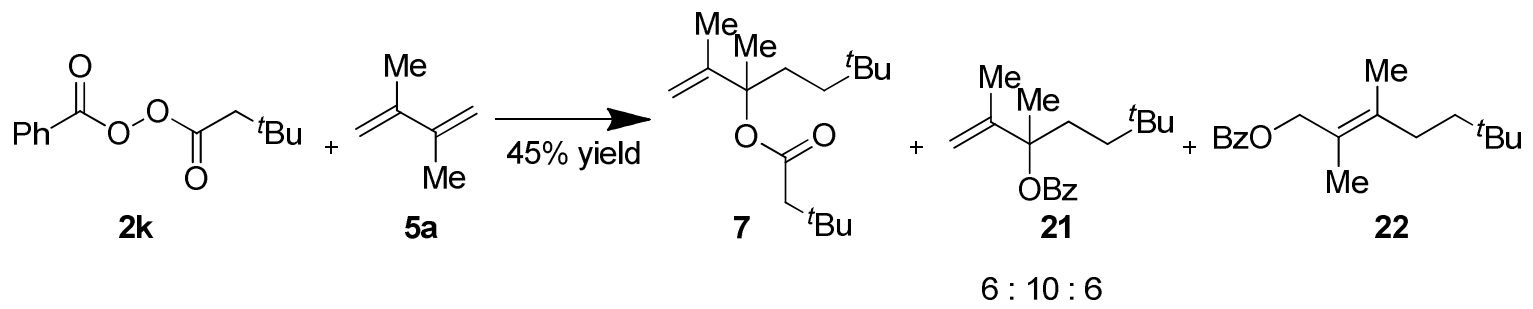

Diacyl peroxide 2k (525 mg, $2.0 \mathrm{mmol}$ ), CuTc (19.1 mg, $0.1 \mathrm{mmol})$, 1,3-diene 5a (246 mg, 3 mmol) and 1,4-dioxane (4 mL) were added into a flame dried schlenk tube with a stirring bar at $70{ }^{\circ} \mathrm{C}$ for 4 hours. Then, the reaction mixture was cooled to ambient temperature, poured into saturated $\mathrm{NaCl}$ solution $(50 \mathrm{~mL})$ and extracted with $\mathrm{CH}_{2} \mathrm{Cl}_{2}(3 \times 50 \mathrm{~mL})$. The combined organic phase was dried over $\mathrm{MgSO}_{4}$. After filtration and evaporation of the solvents under reduced pressure, the crude product was purified by column chromatography on silica gel (petroleum ether/ethyl acetate $=100 / 0 \sim 20 / 1)$ to yield $45 \%$ of the mixed product 7,21 and $22(\mathbf{7}: \mathbf{2 1}: \mathbf{2 2}=6$ : $10: 6)$.<smiles>C=C(C)C(C)(CCCBr)OC(=O)OCc1ccccc1</smiles>

21

${ }^{1} \mathrm{H} \mathrm{NMR}\left(400 \mathrm{MHz}, \mathrm{CDCl}_{3}\right) \delta 8.03(\mathrm{~d}, J=8.00 \mathrm{~Hz}, 2 \mathrm{H}), 7.54(\mathrm{~d}, J=8.00 \mathrm{~Hz}, 1 \mathrm{H}), 7.43(\mathrm{~d}, J=$ $8.00 \mathrm{~Hz}, 2 \mathrm{H}), 4.99(\mathrm{~d}, J=8.00 \mathrm{~Hz}, 2 \mathrm{H}), 1.98-1.84(\mathrm{~m}, 2 \mathrm{H}), 1.76(\mathrm{~s}, 3 \mathrm{H}), 1.71(\mathrm{~s}, 3 \mathrm{H}), 1.21-1.17$ $(\mathrm{m}, 2 \mathrm{H}), 0.90(\mathrm{~s}, 9 \mathrm{H}) .{ }^{13} \mathrm{C} \mathrm{NMR}\left(100 \mathrm{MHz}, \mathrm{CDCl}_{3}\right) \delta 165.10,146.91,132.57,131.63,129.46$, 128.28, 111.30, 86.09, 37.37, 33.72, 29.35, 23.08, 19.00. IR (thin film): 3090, 2928, 2852, 1718, $1450,1313,1279,1712,1108,1086,1024,899,712 \mathrm{~cm}^{-1}$. HRMS (ESI) calcd for $\left[\mathrm{C}_{18} \mathrm{H}_{26} \mathrm{NaO}_{2}\right]^{+}$ $\left([\mathrm{M}+\mathrm{Na}]^{+}\right): 297.1825$, found: 297.1827 . 
S-15

NMR Spectra

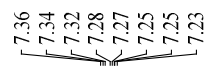

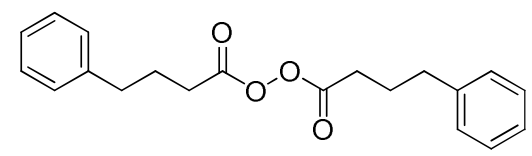

$2 b$
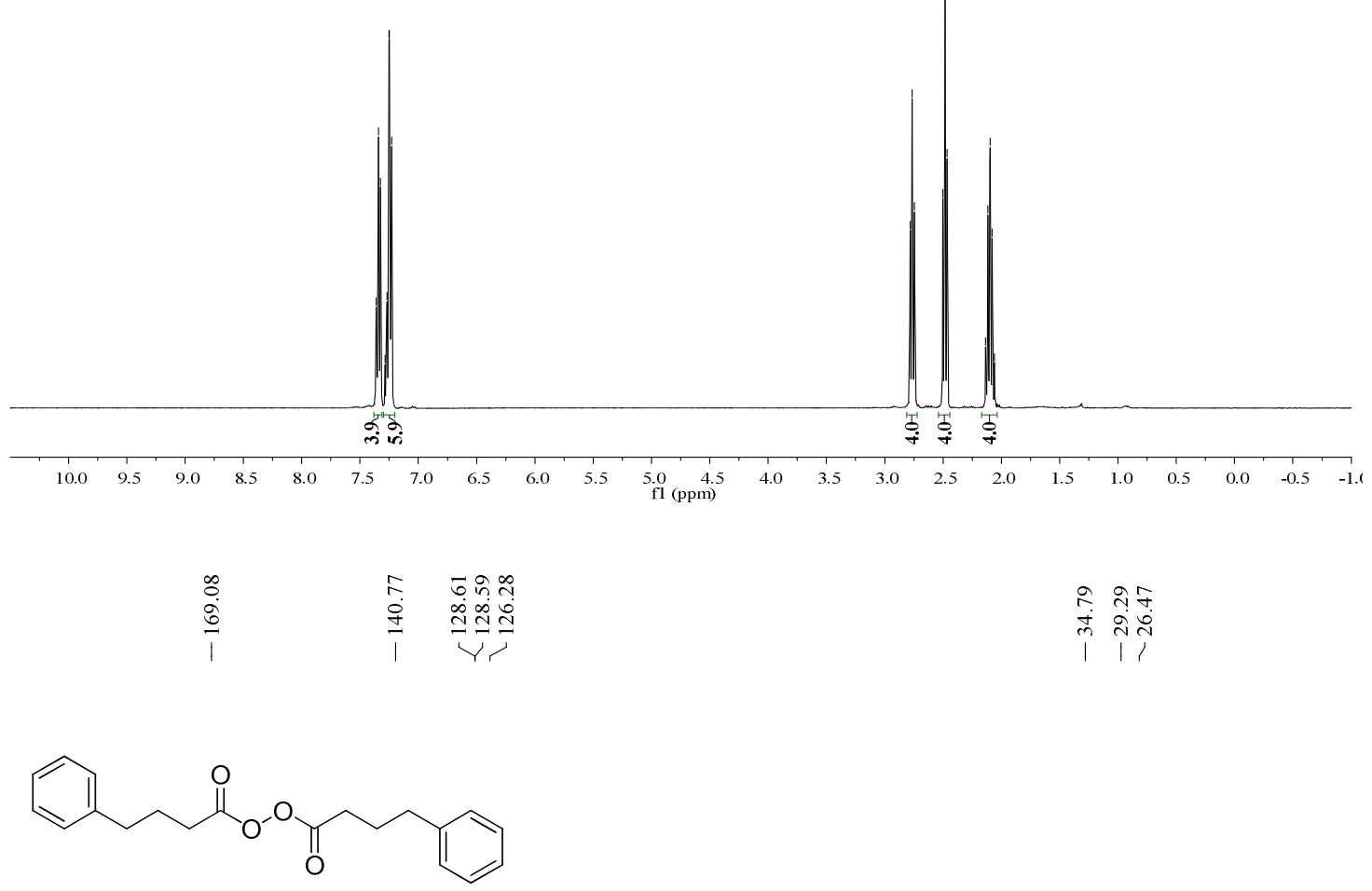

ab

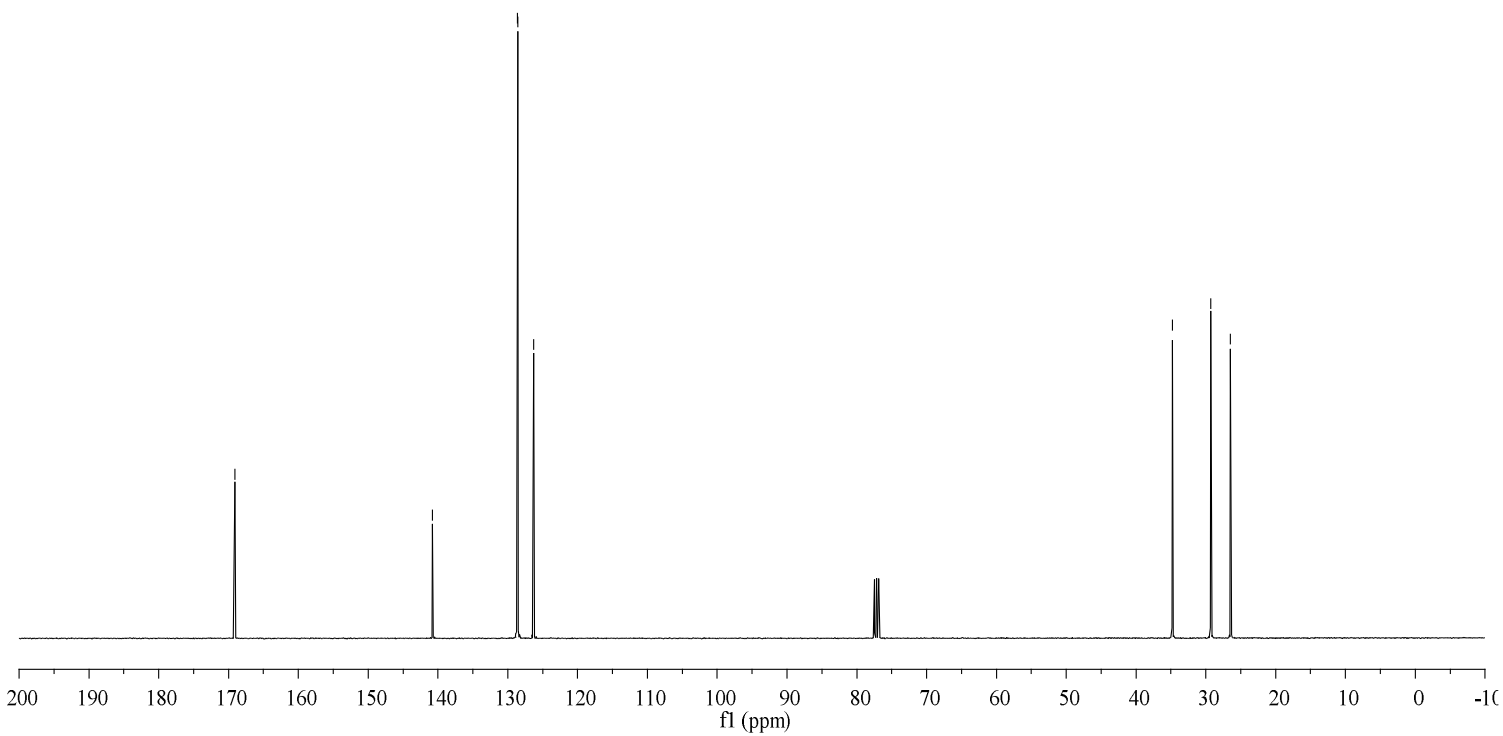



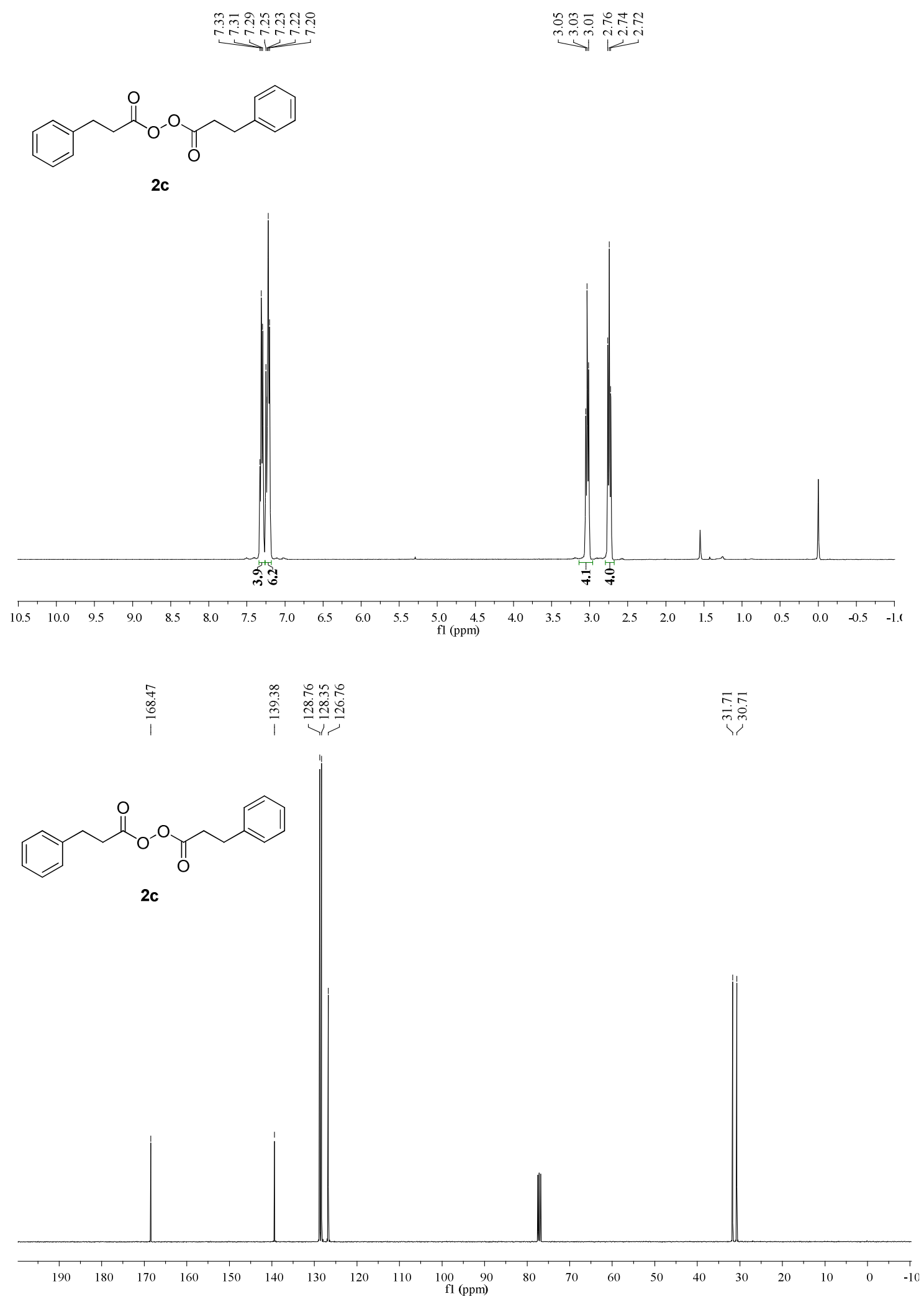


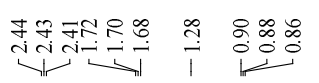

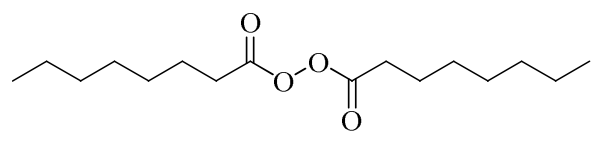

2d

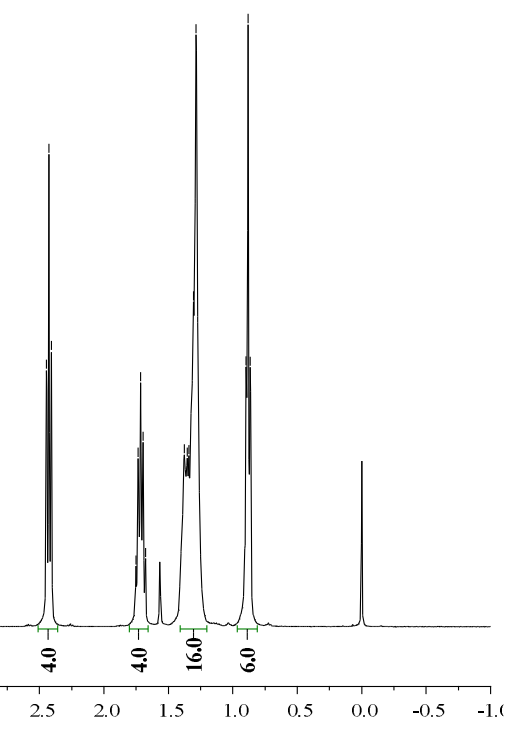

ปี

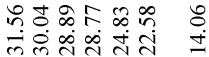

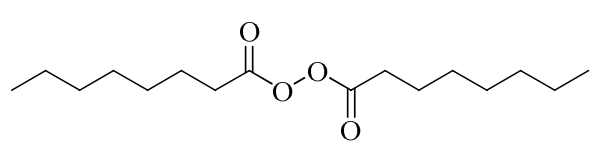

2d

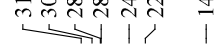

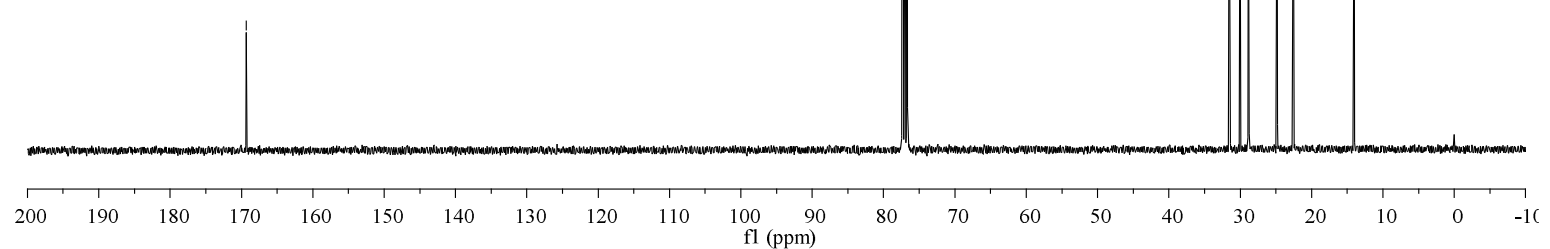




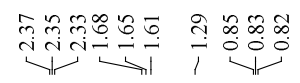

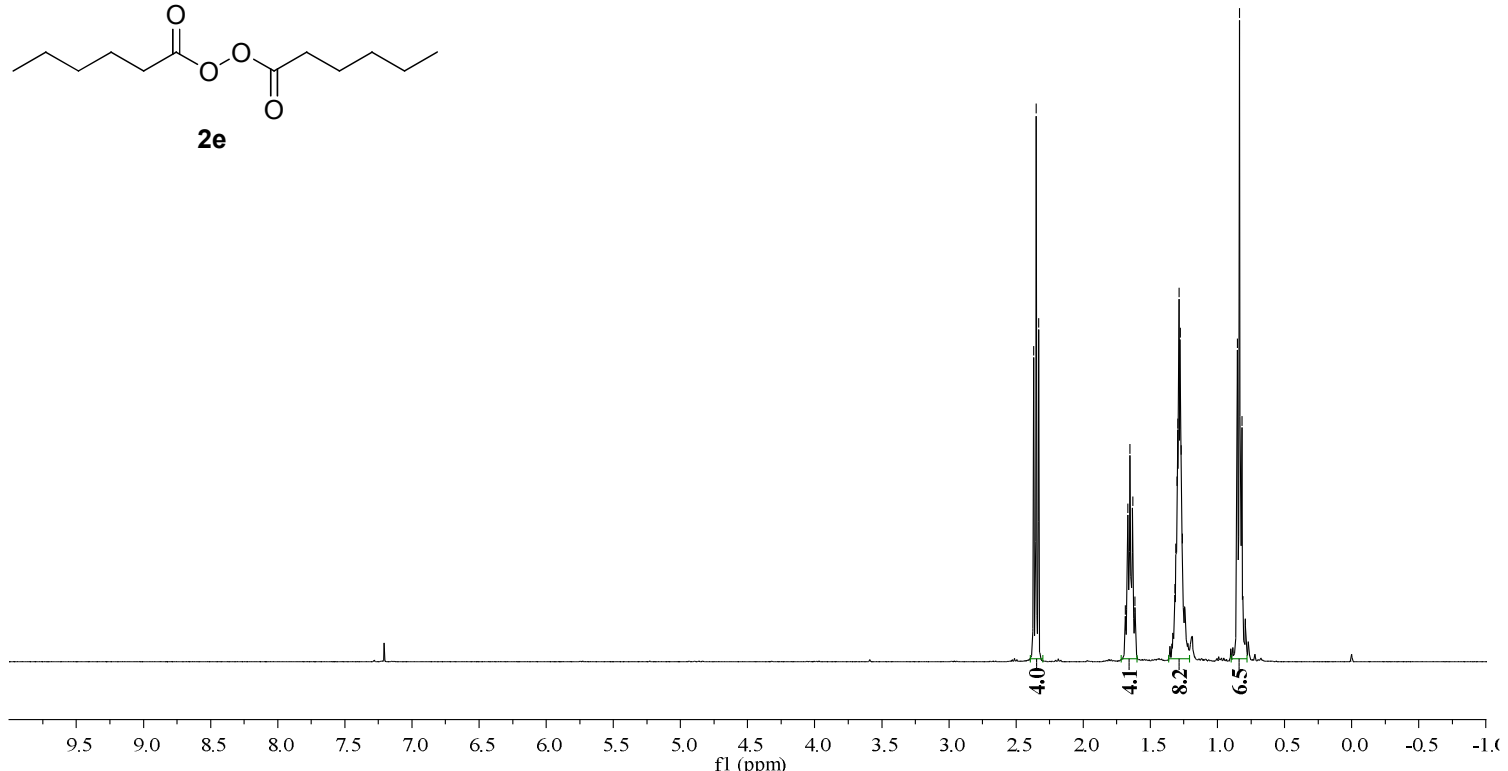

章

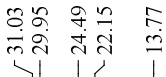

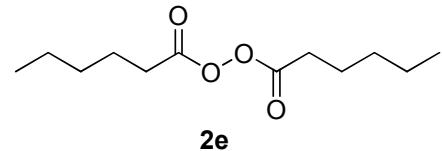

$2 e$

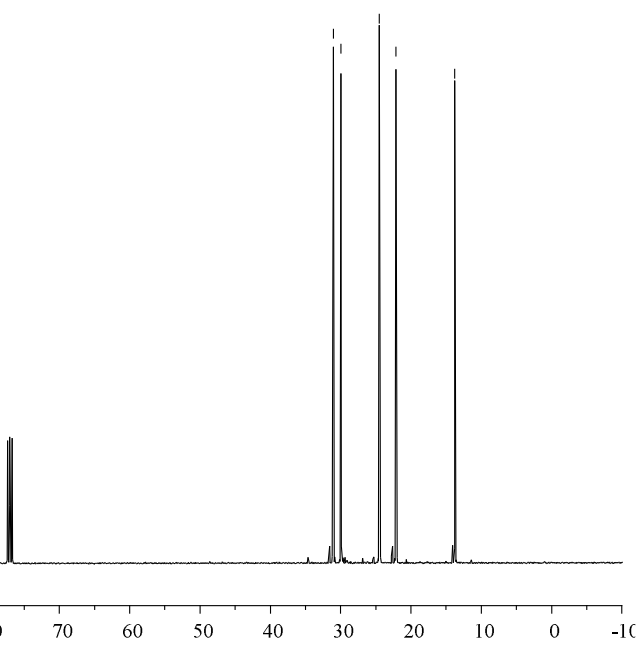


S-19

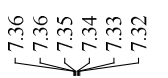

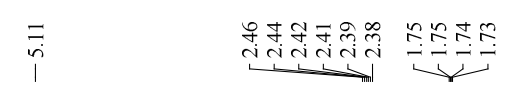

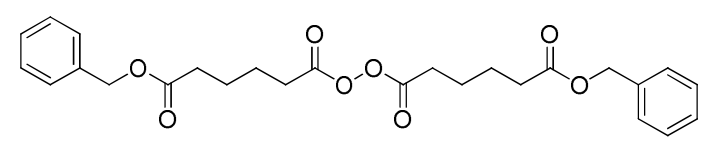

$2 \mathbf{f}$

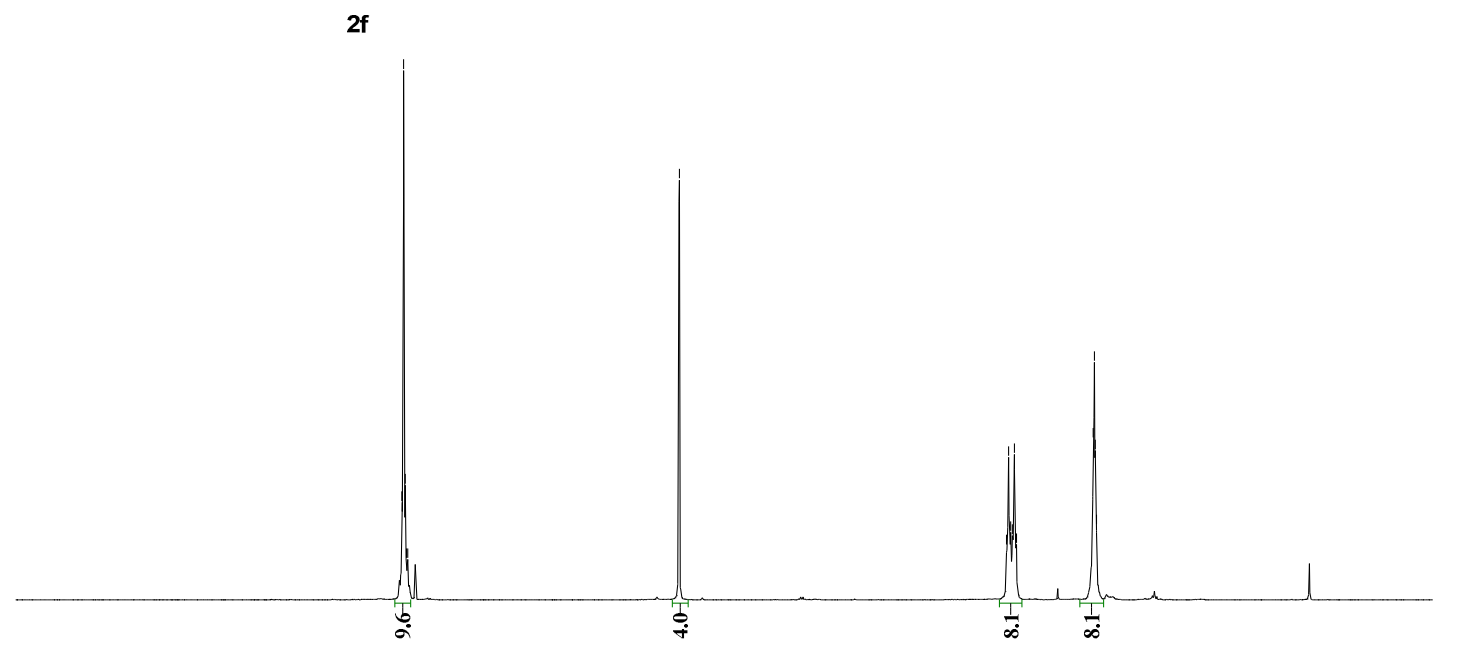

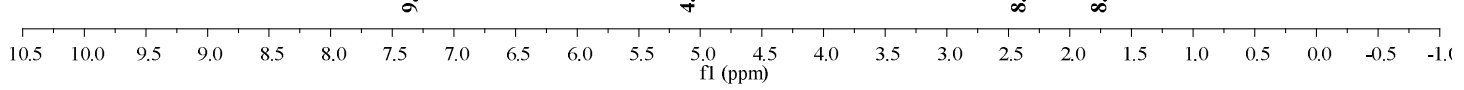

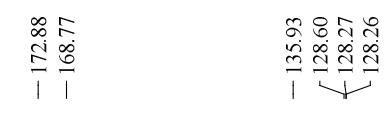

$\overline{3}$
i
$i$

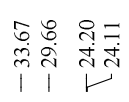
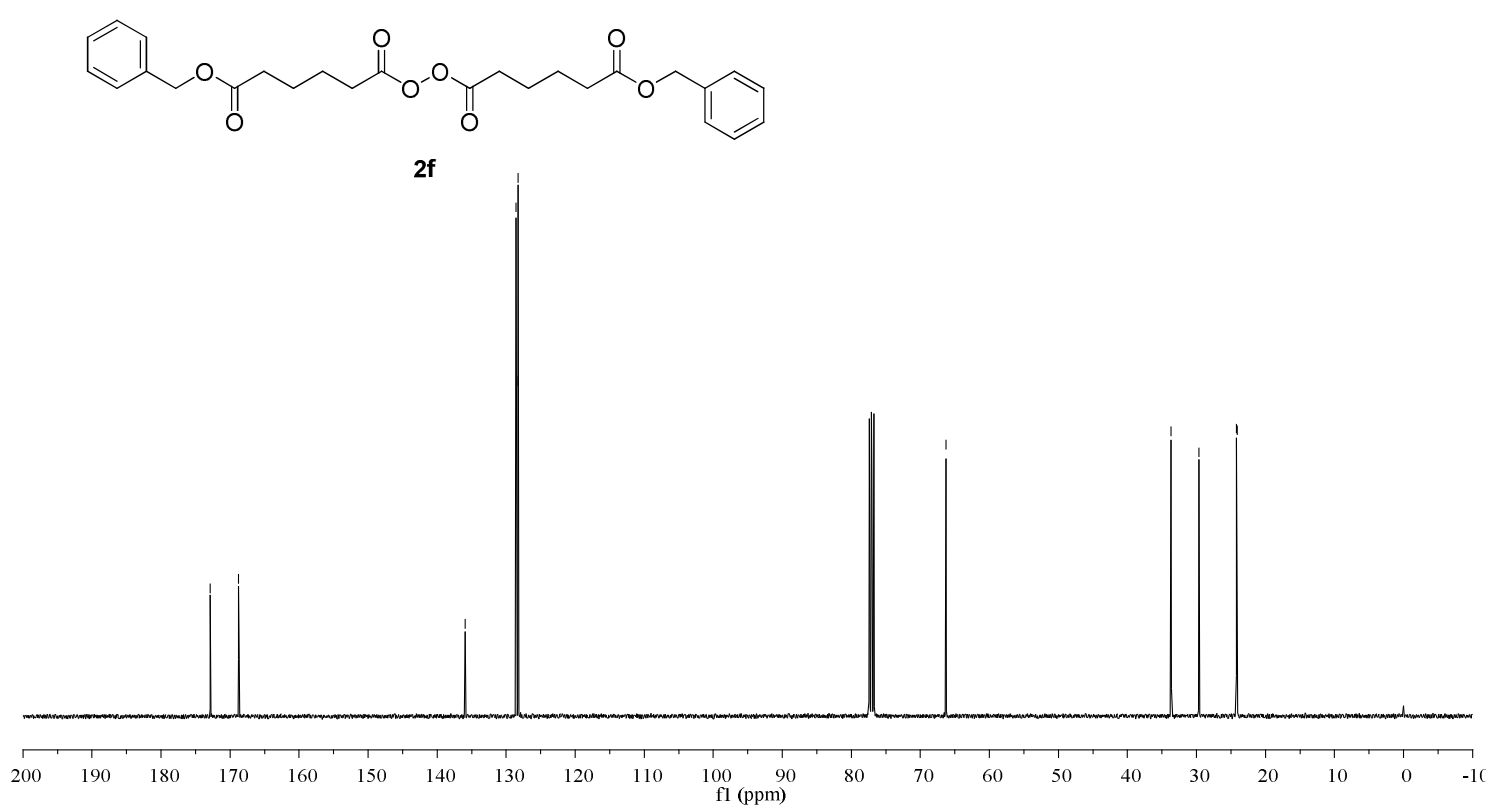
S-20

$\stackrel{3}{1} \stackrel{?}{1}$

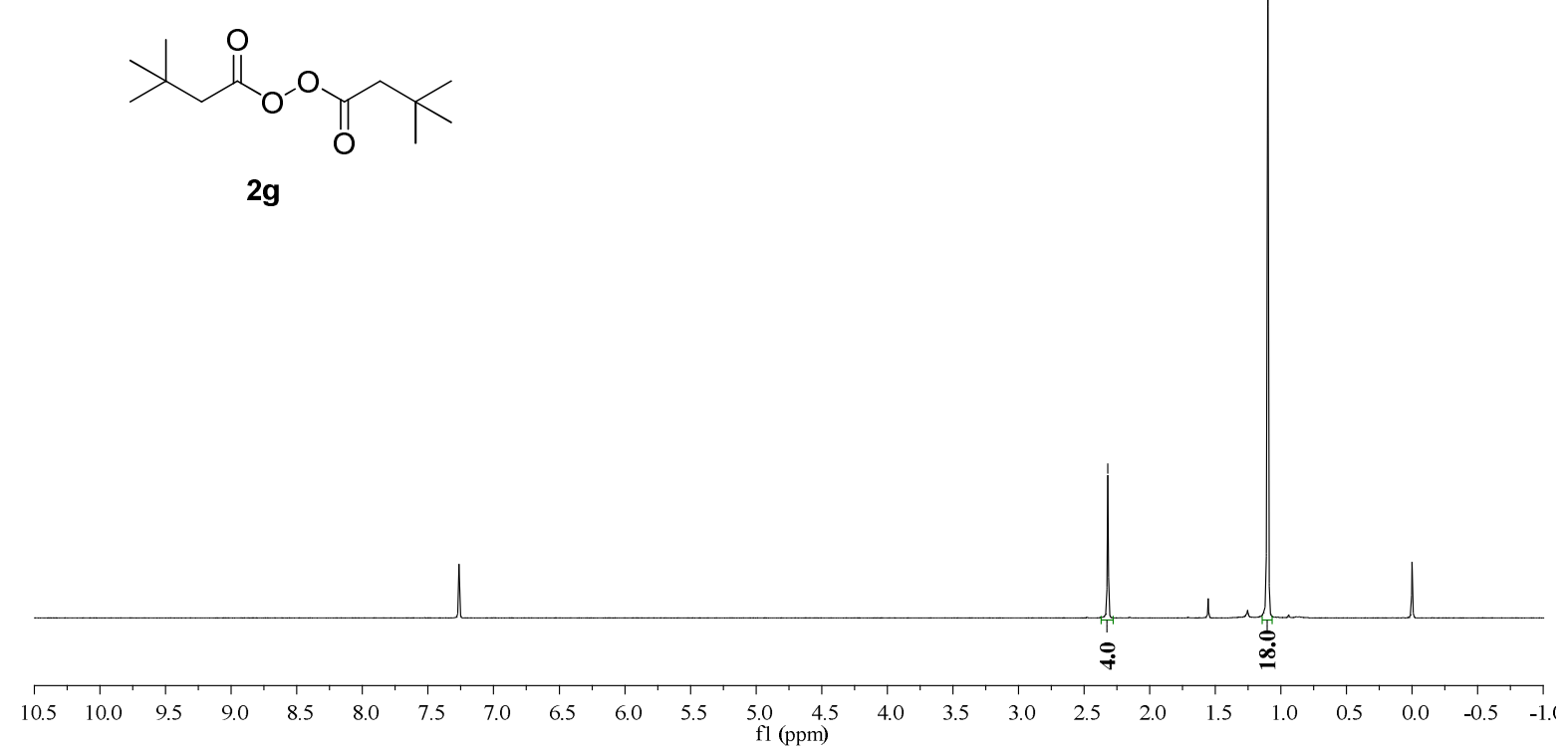

$2 g$

$\frac{1}{\frac{1}{0}}$

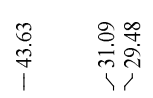

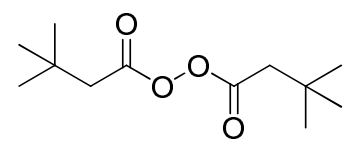

$2 g$

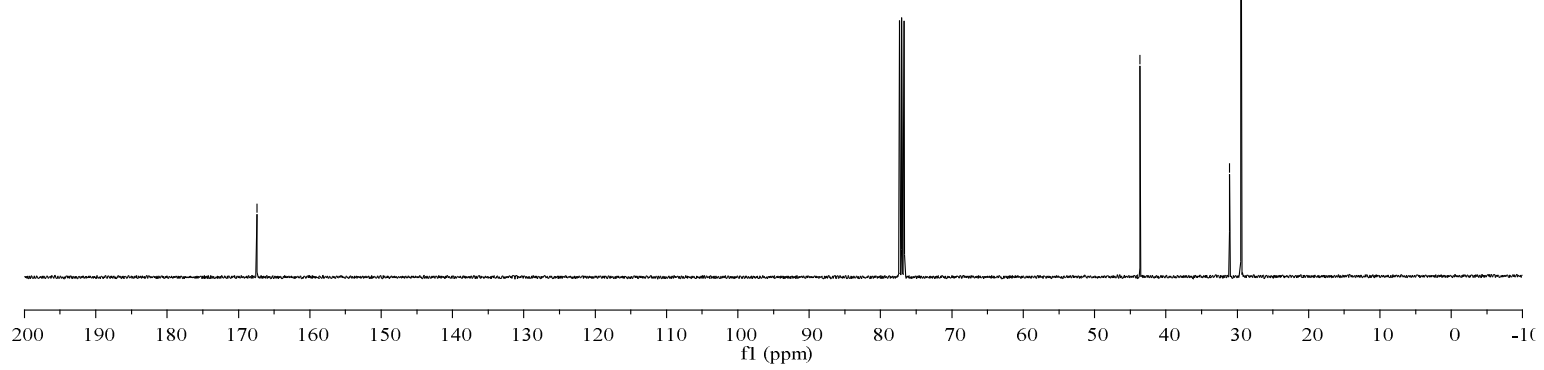




\section{त)}<smiles>C=CCCC(=O)OOC(=O)CCC=C</smiles>

2h

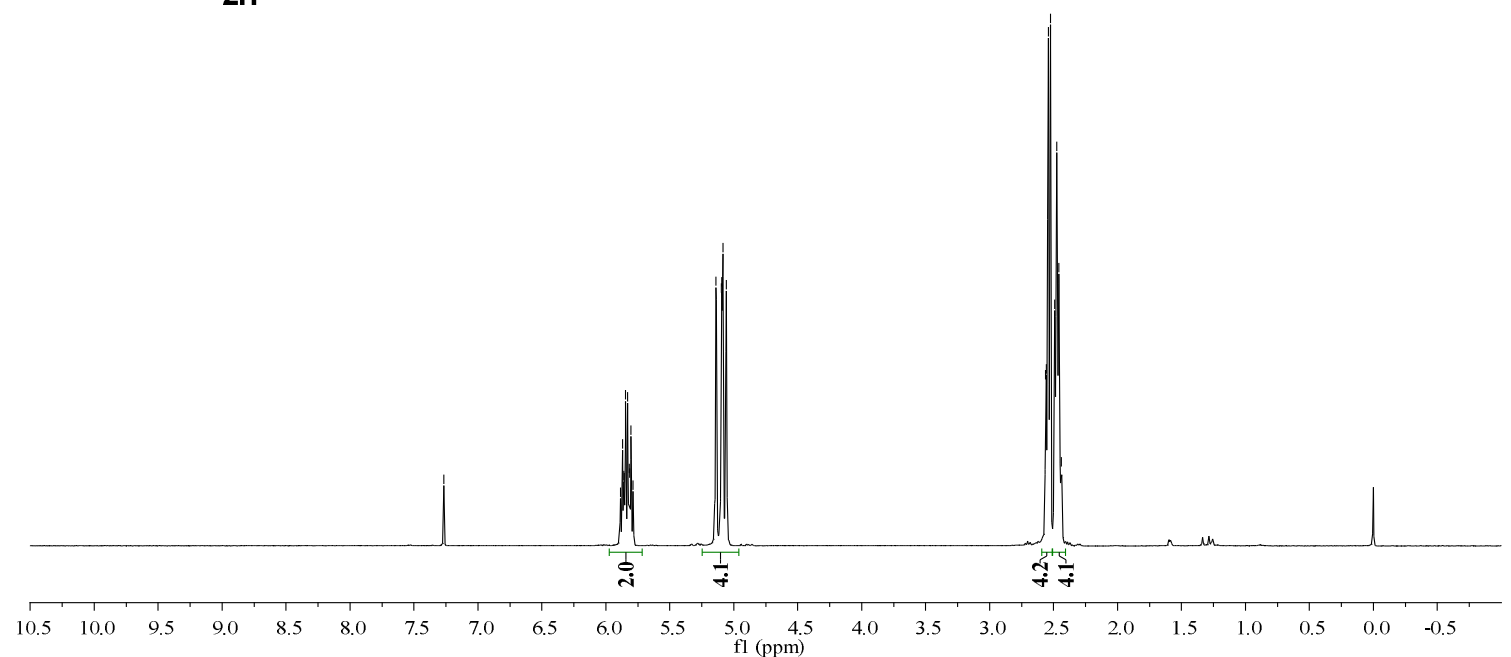

ind<smiles>C=CCCC(=O)OOC(=O)CCC=C</smiles>

2h

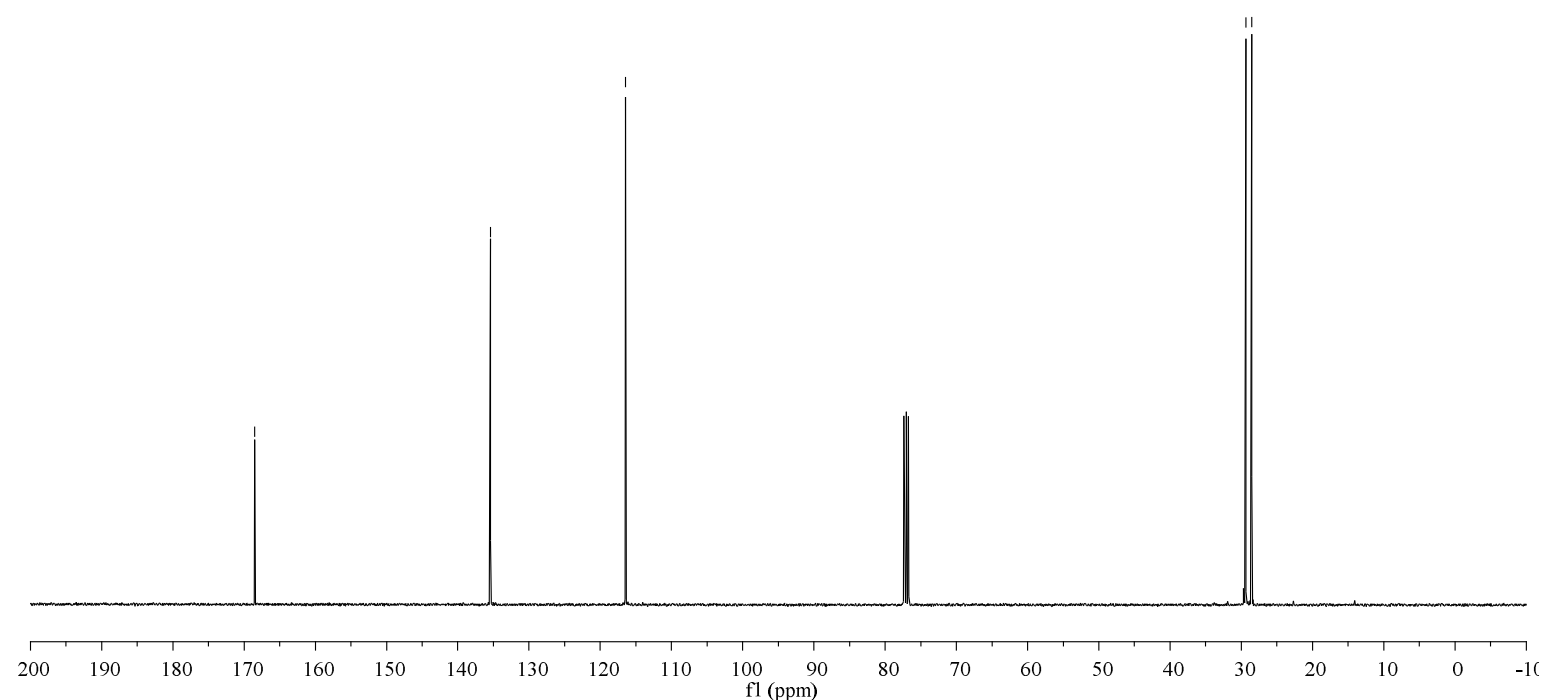



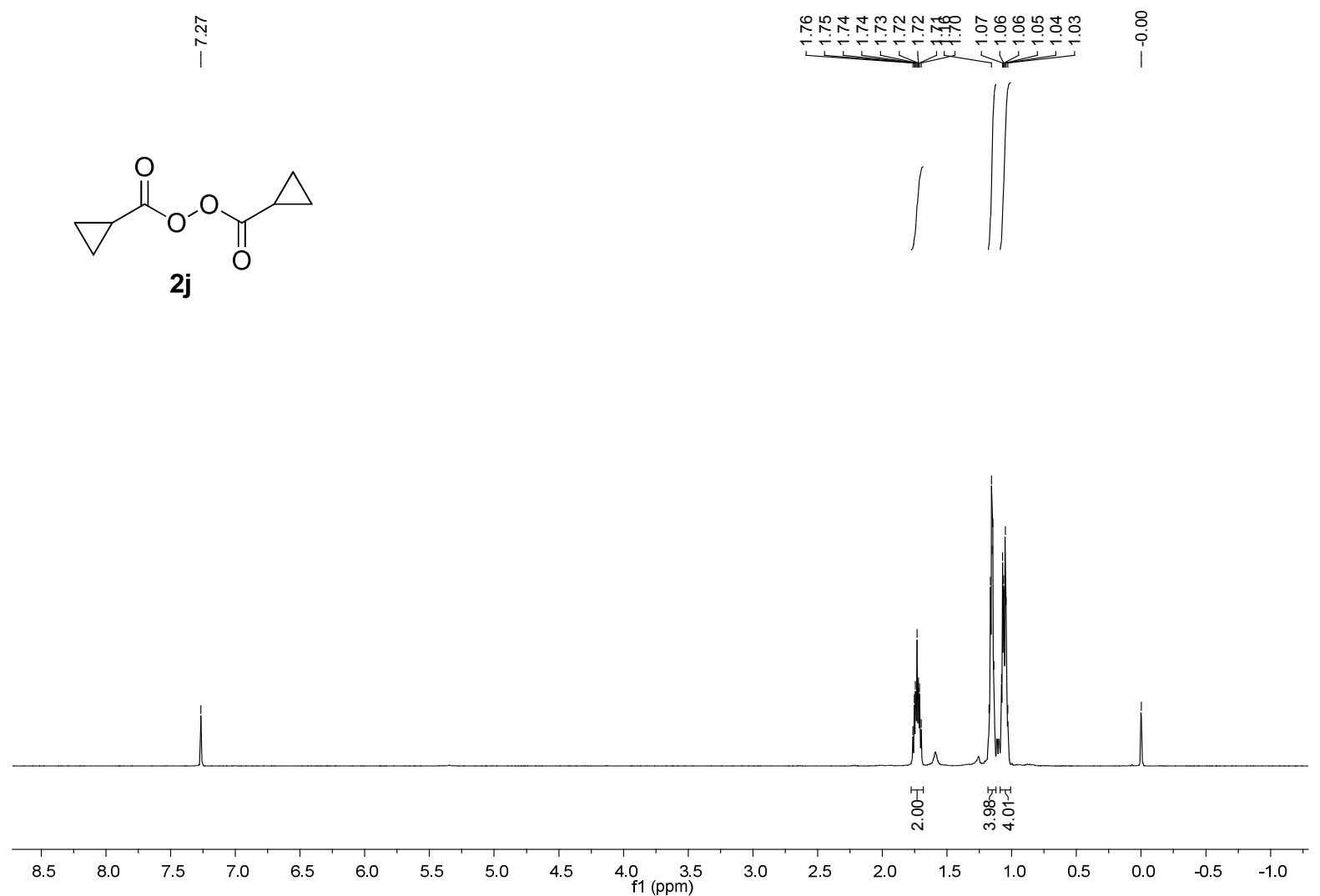

$\frac{\circ}{1}$

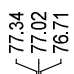

Y̛<smiles>O=C(OOC(=O)C1CC1)C1CC1</smiles>

2j

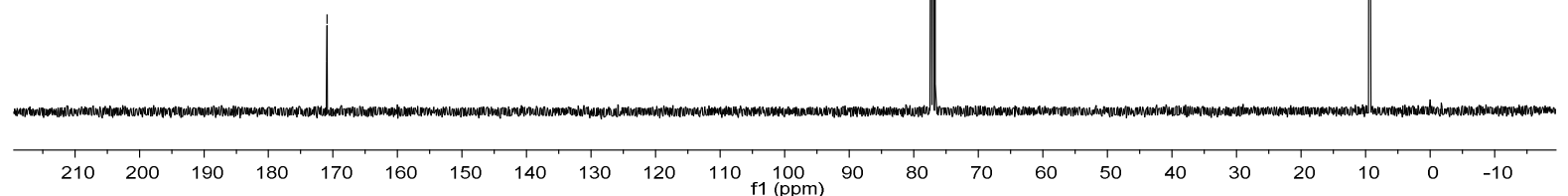




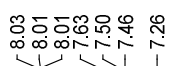

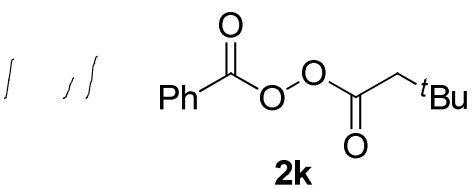

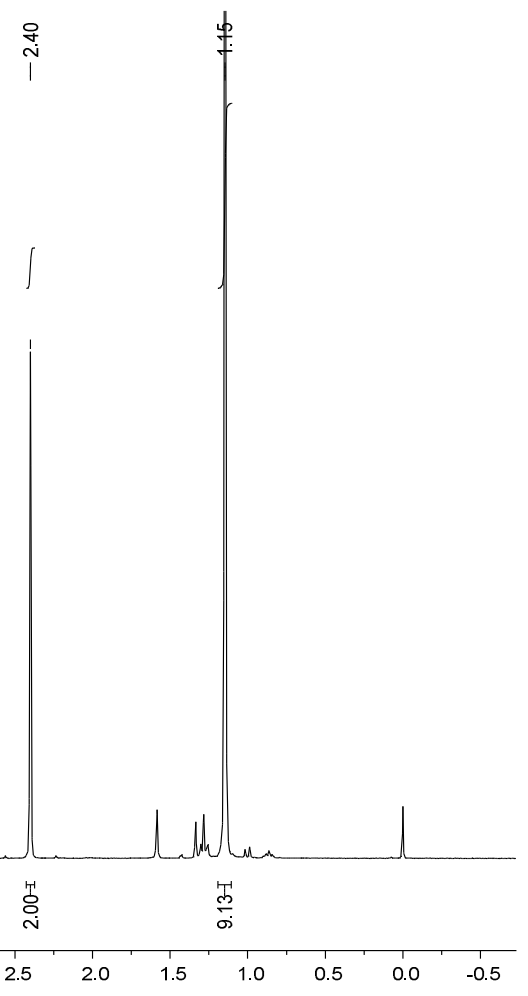

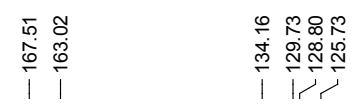

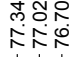

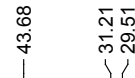<smiles>CC(C)(C)CC(=O)OOC(=O)c1ccccc1</smiles>

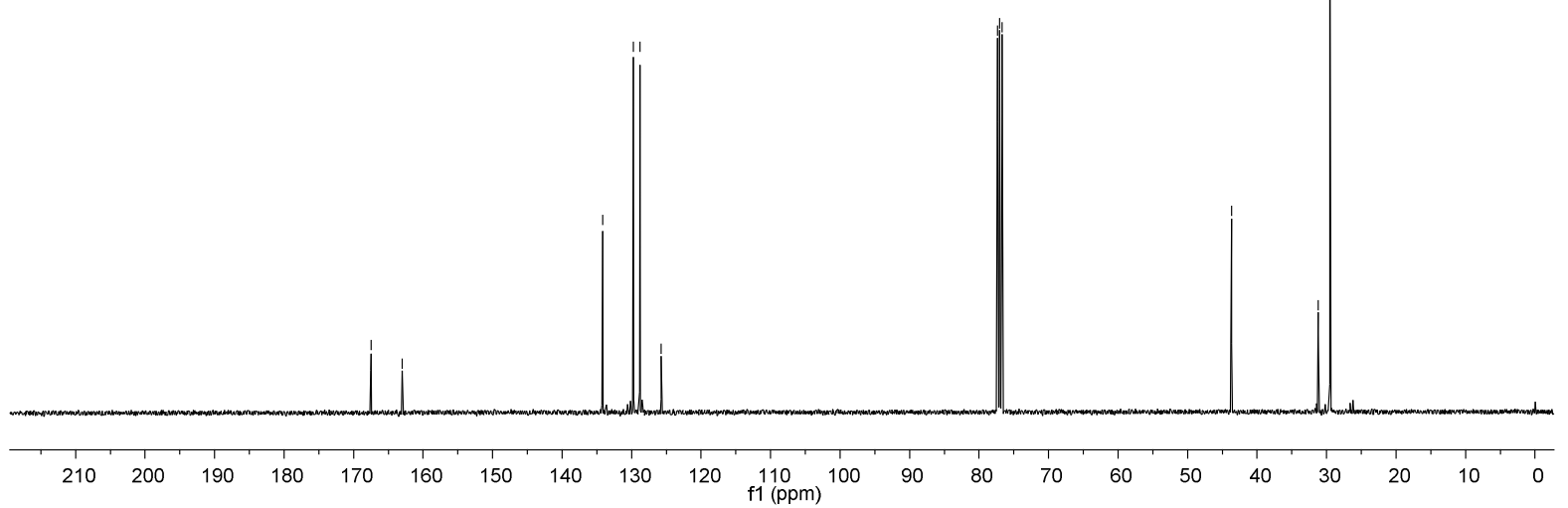



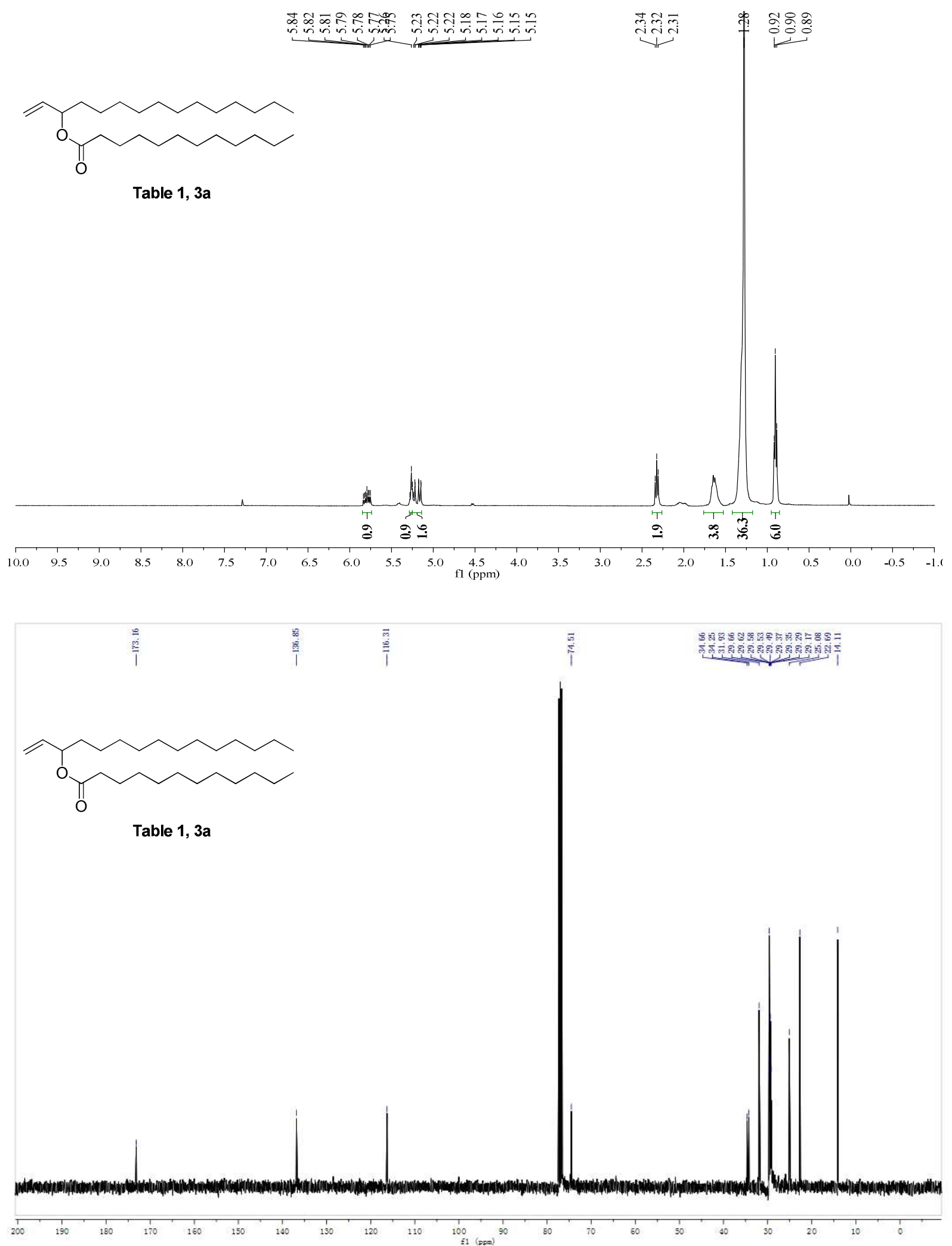


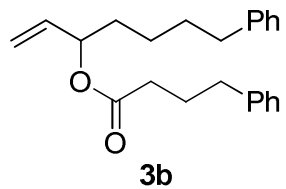

Table 2, entry 1
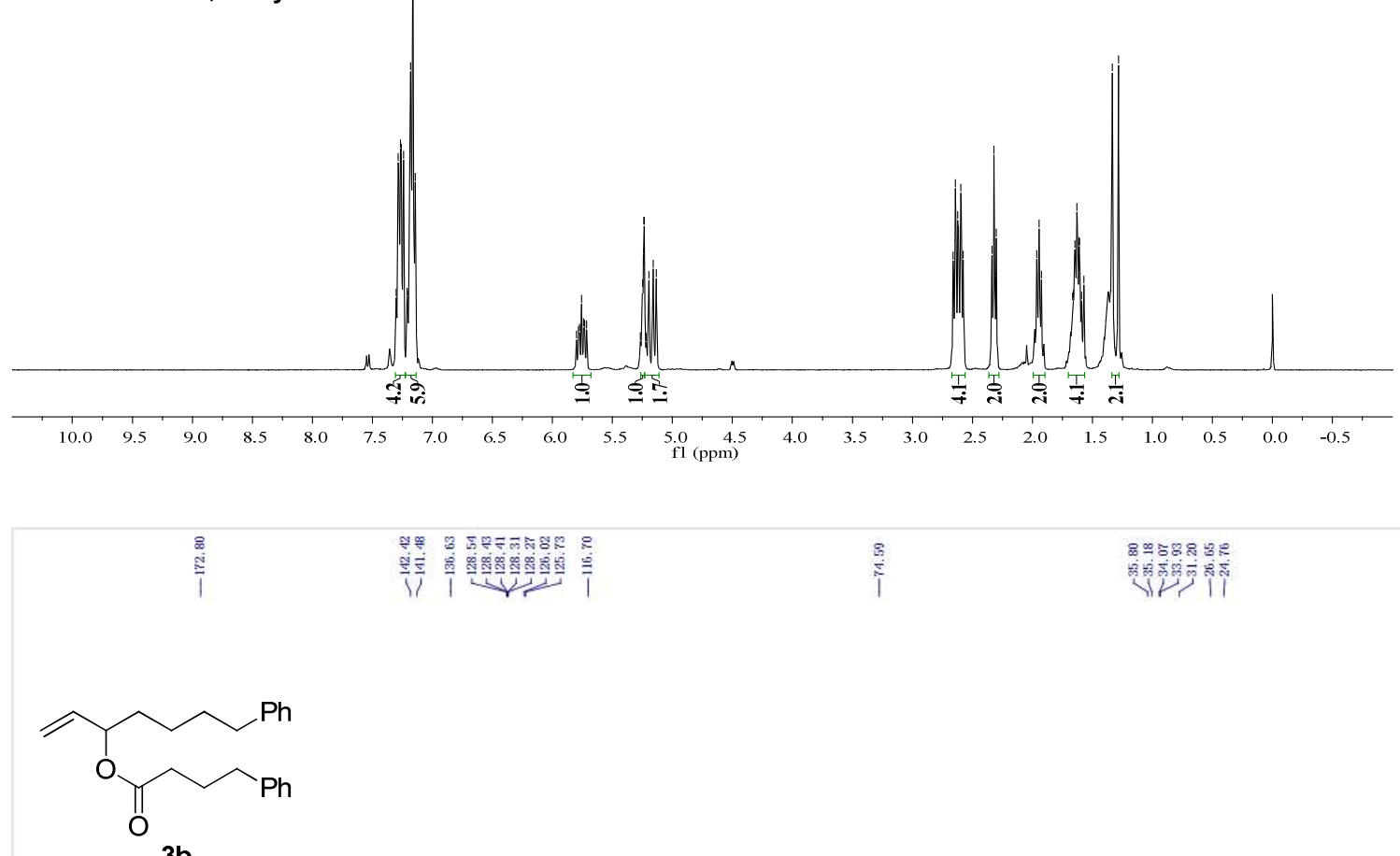

$3 b$

Table 2, entry 1
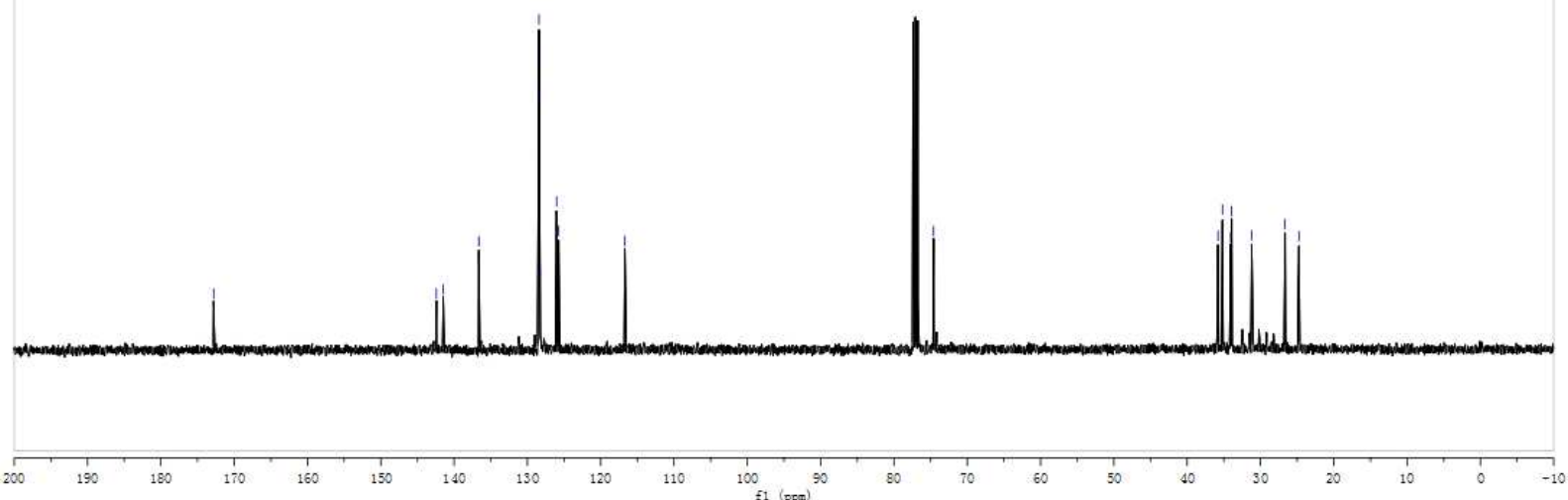


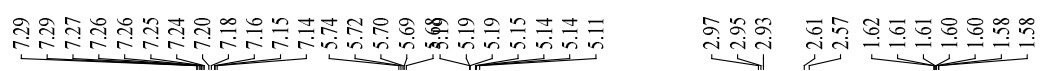<smiles>C=CC(CCCc1ccccc1)OC(=O)CCc1ccccc1</smiles>

Table 2, entry 2
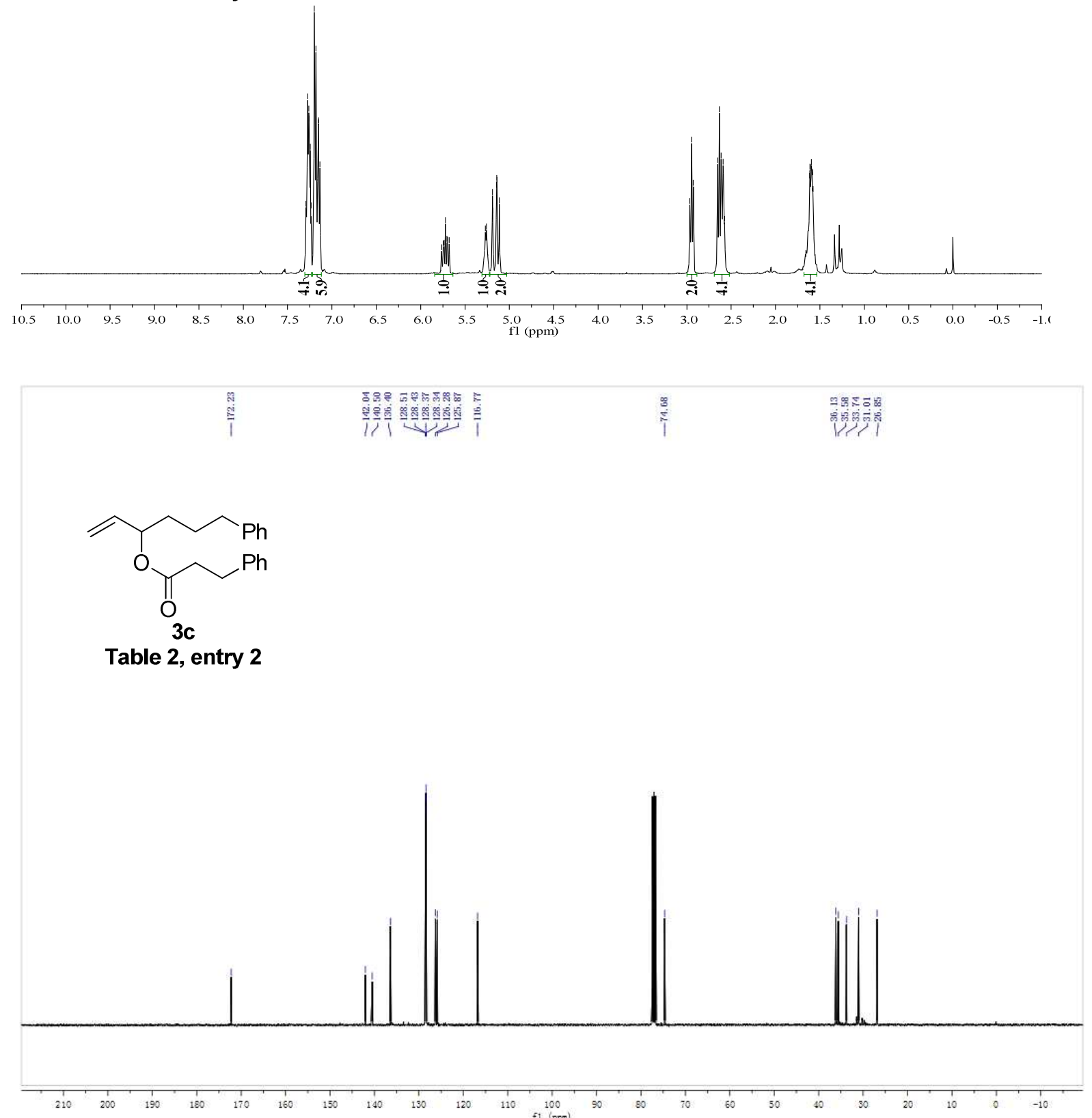


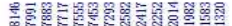

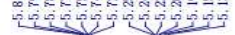

$\overbrace{\mathrm{O}}^{\mathrm{C}_{7} \mathrm{H}_{15}} \mathrm{C}_{7} \mathrm{H}_{15}$

Table 2, entry 3

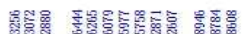

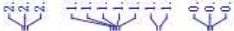
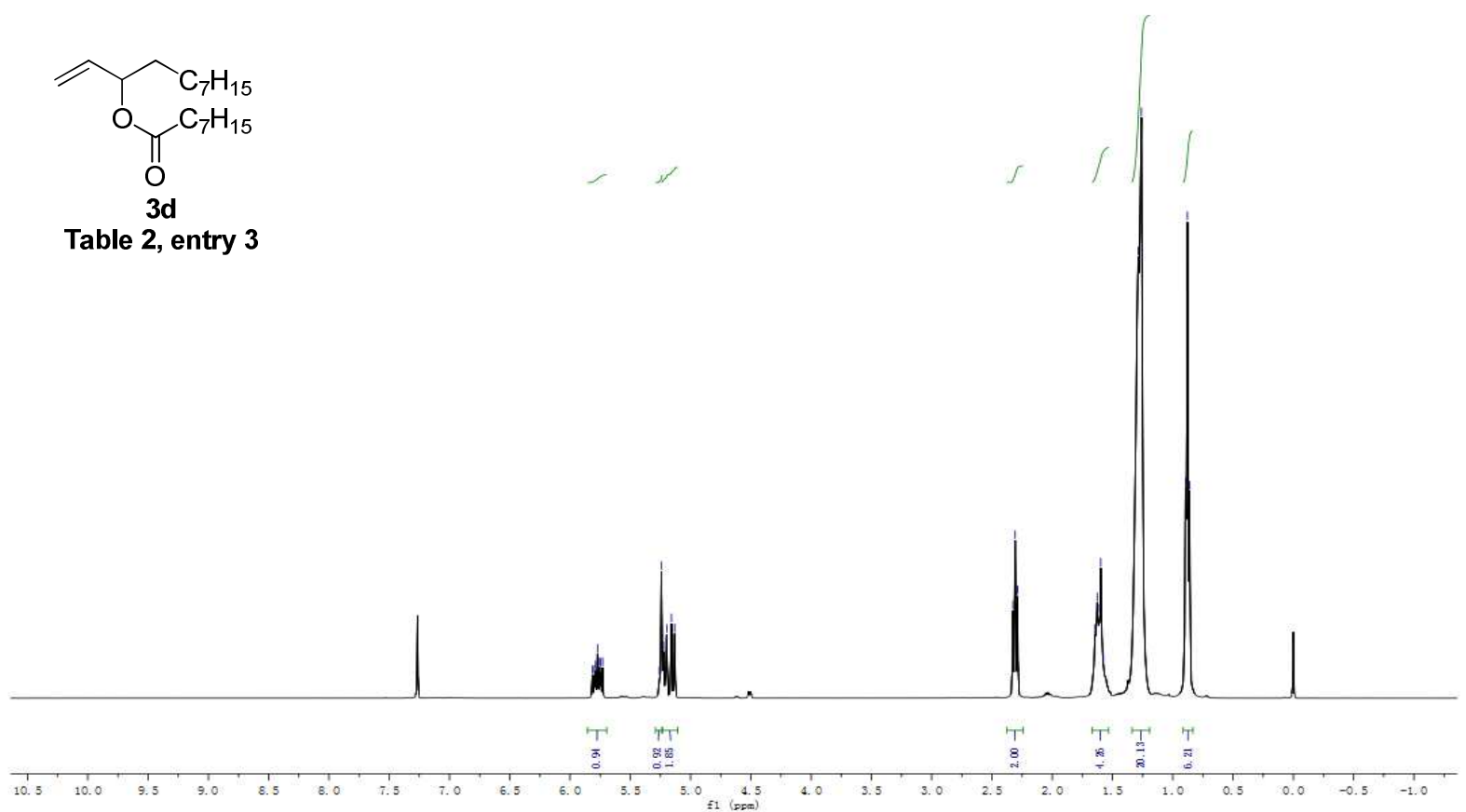

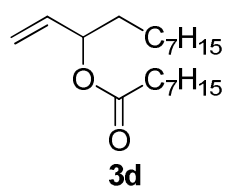

Table 2, entry 3
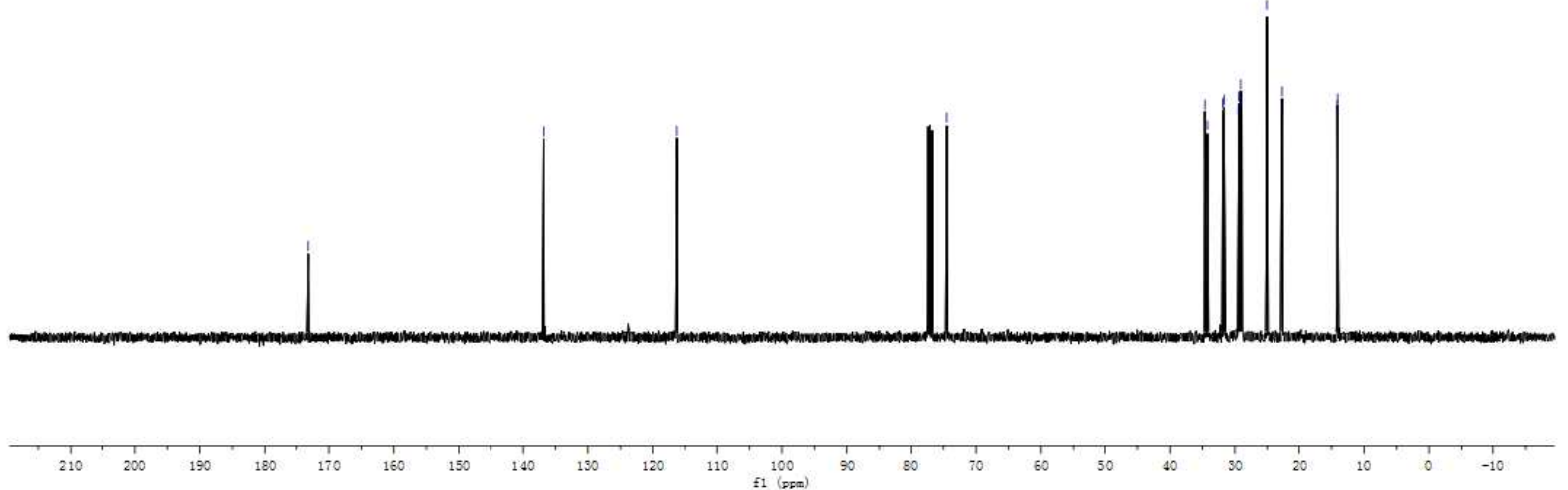


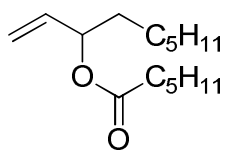

$3 e$

Table 2, entry 4

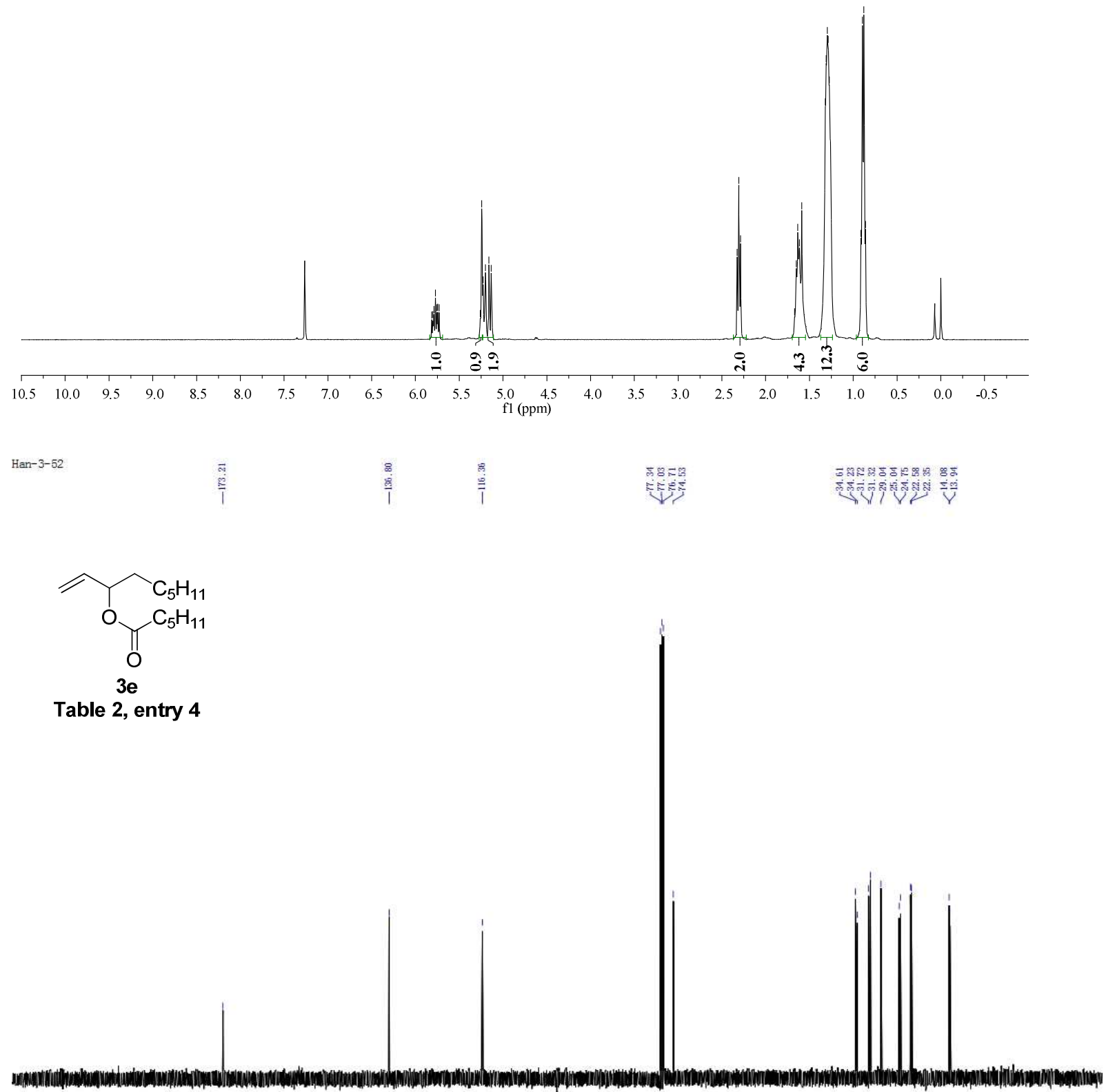

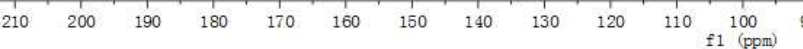




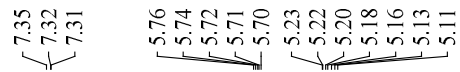

$\underbrace{\infty}$

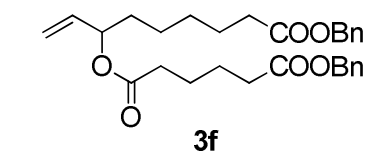

Table 2, entry 5

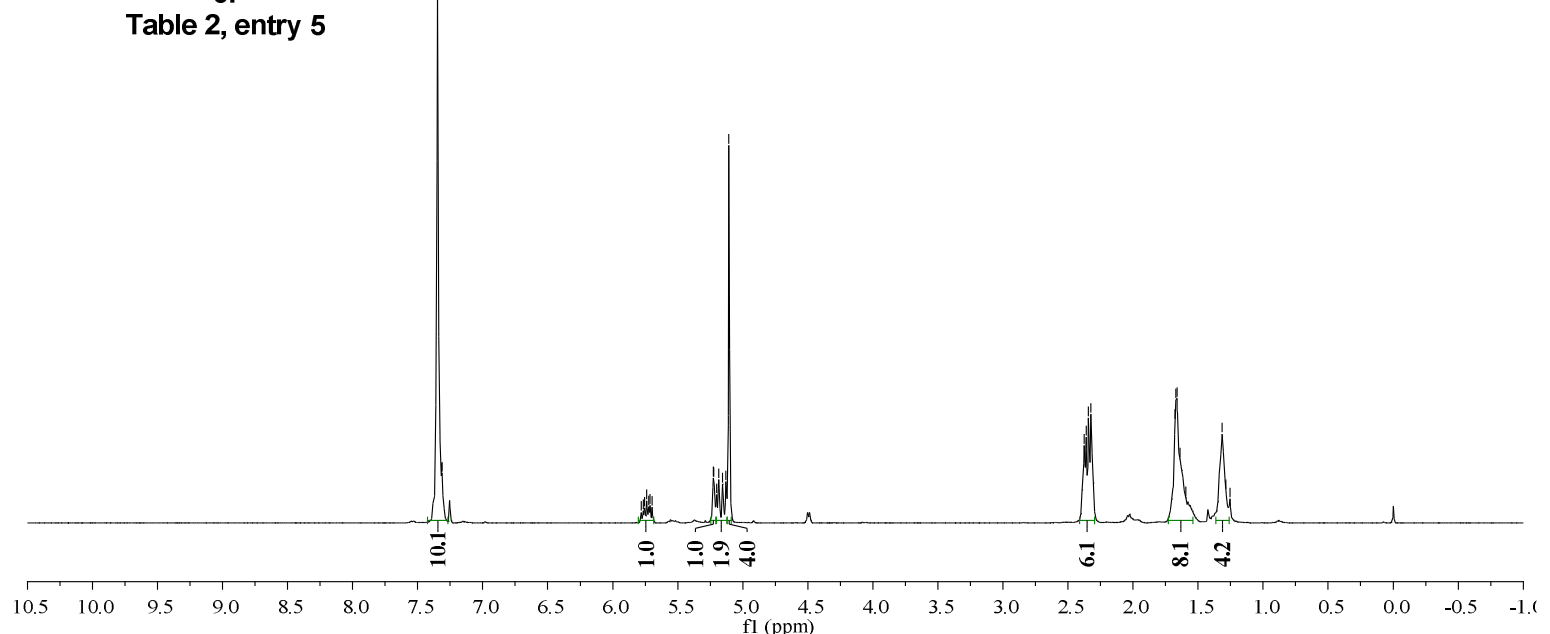

Table 2, entry 5

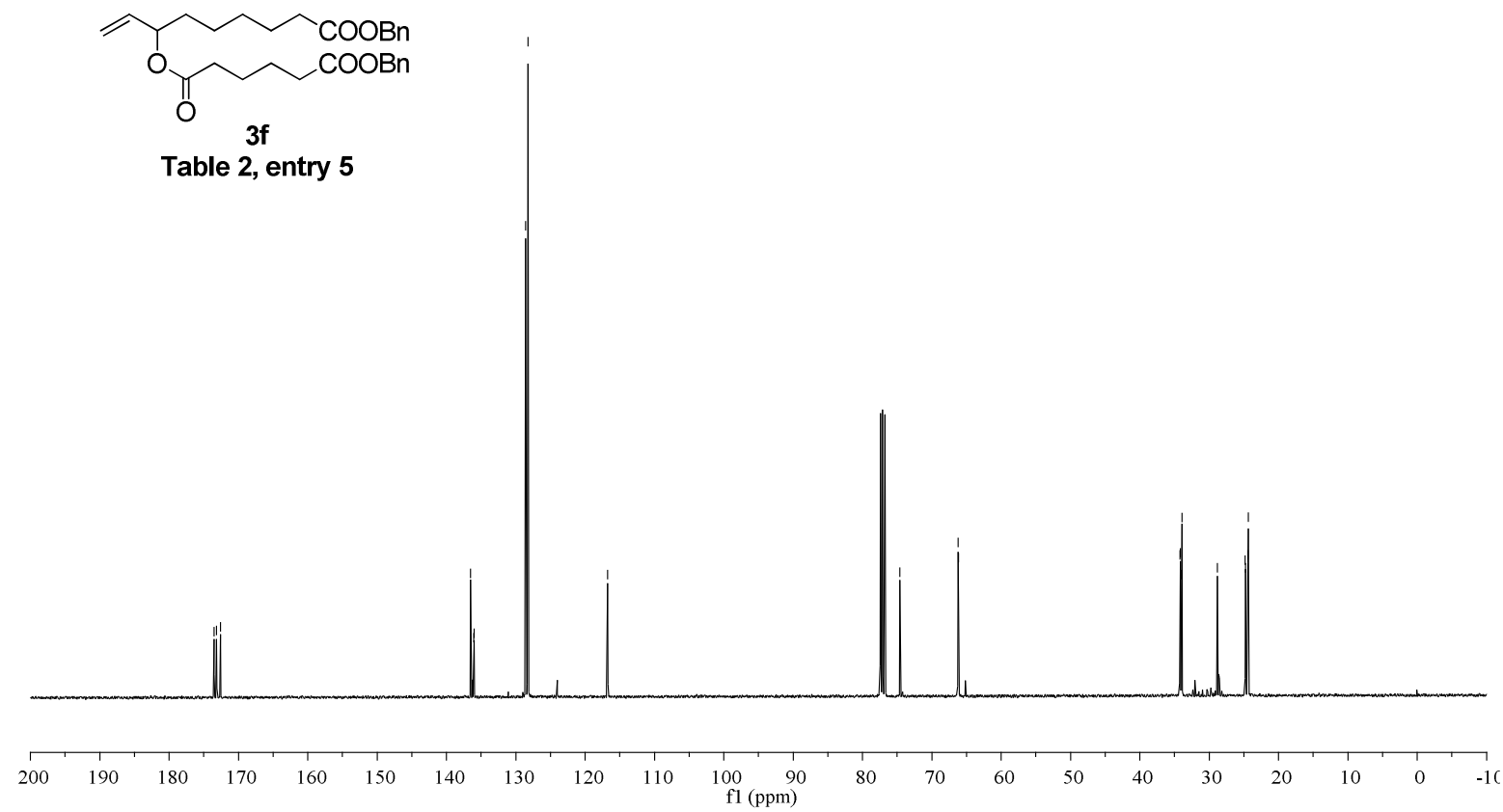




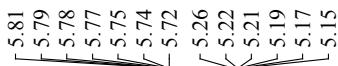

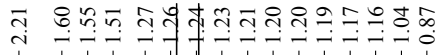<smiles>C=CC(CCC(C)(C)C)OC(=O)CC(C)(C)C</smiles>

Table 2, entry 6

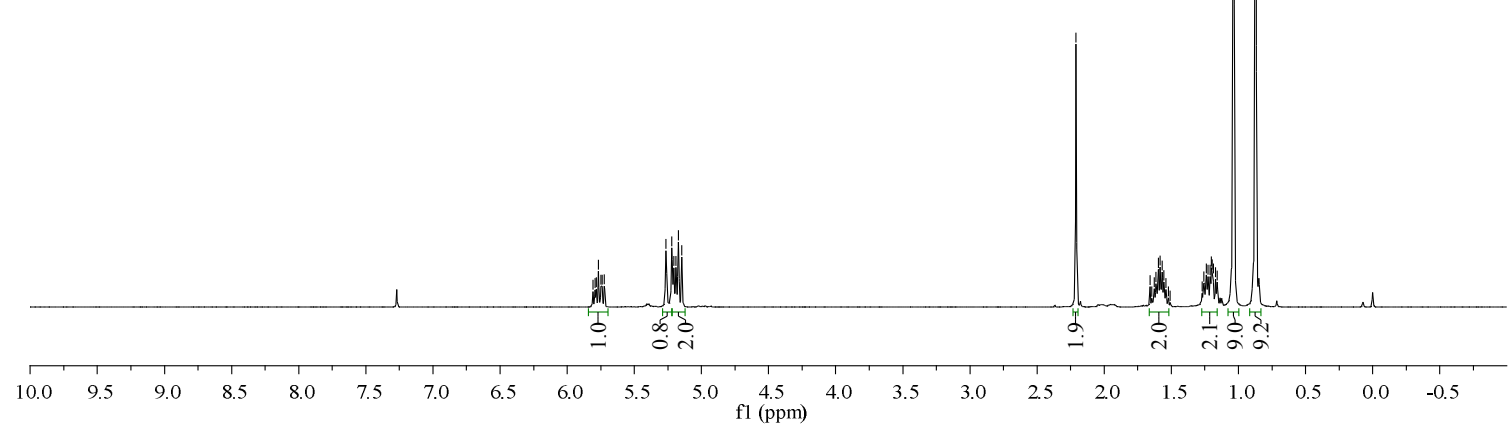

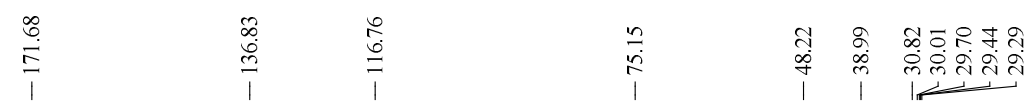

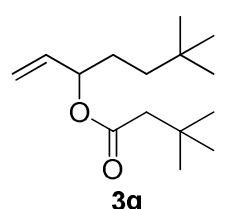

Table 2, entry 6

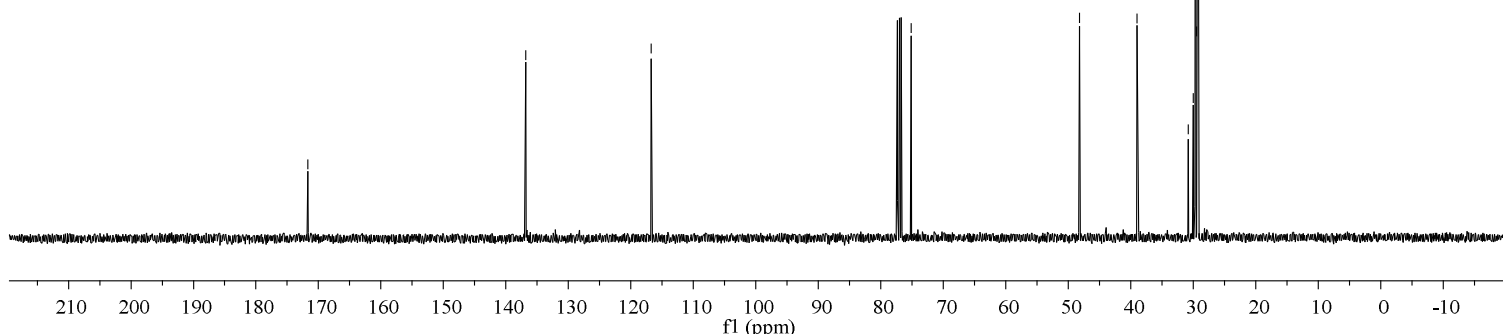




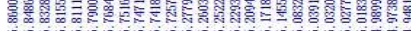

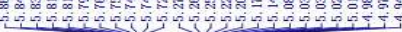

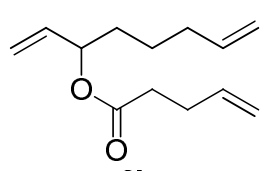

$3 \mathrm{~h}$

Table 2, entry 7

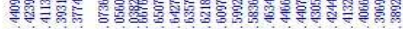

Nis

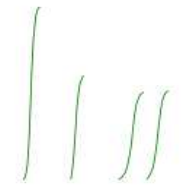

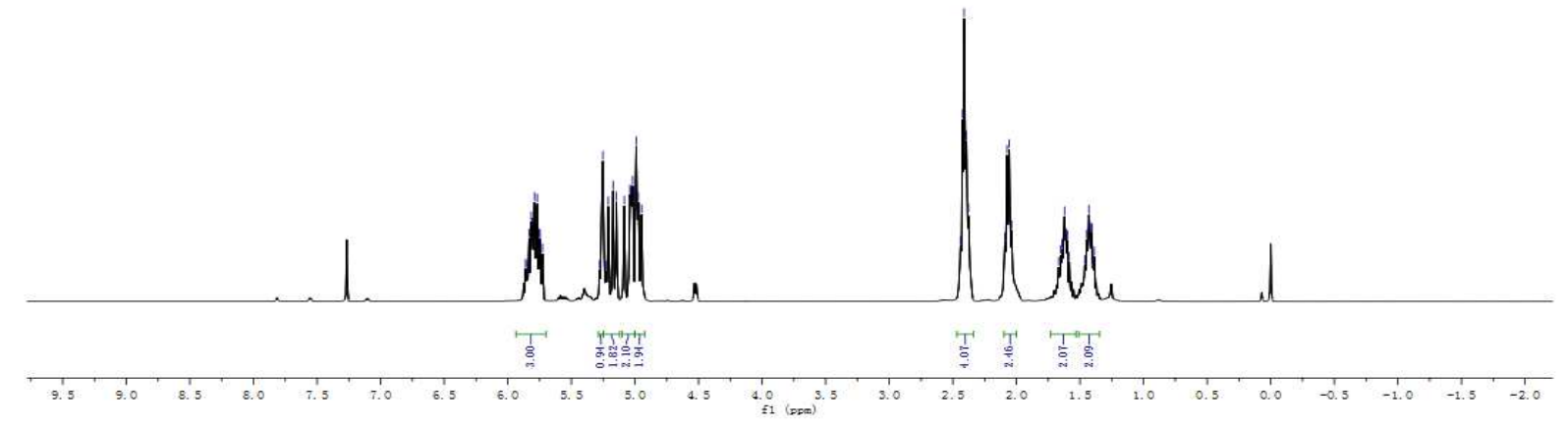

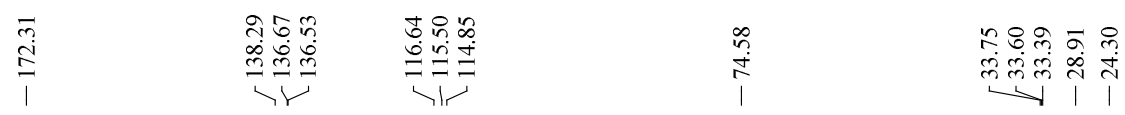

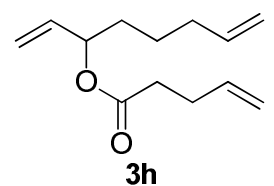

Table 2, entry 7

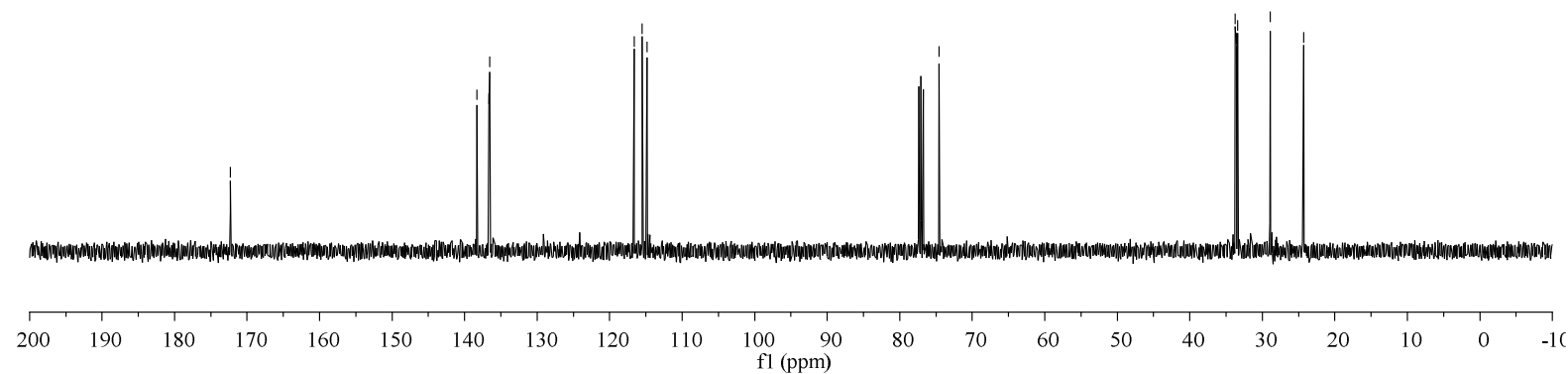




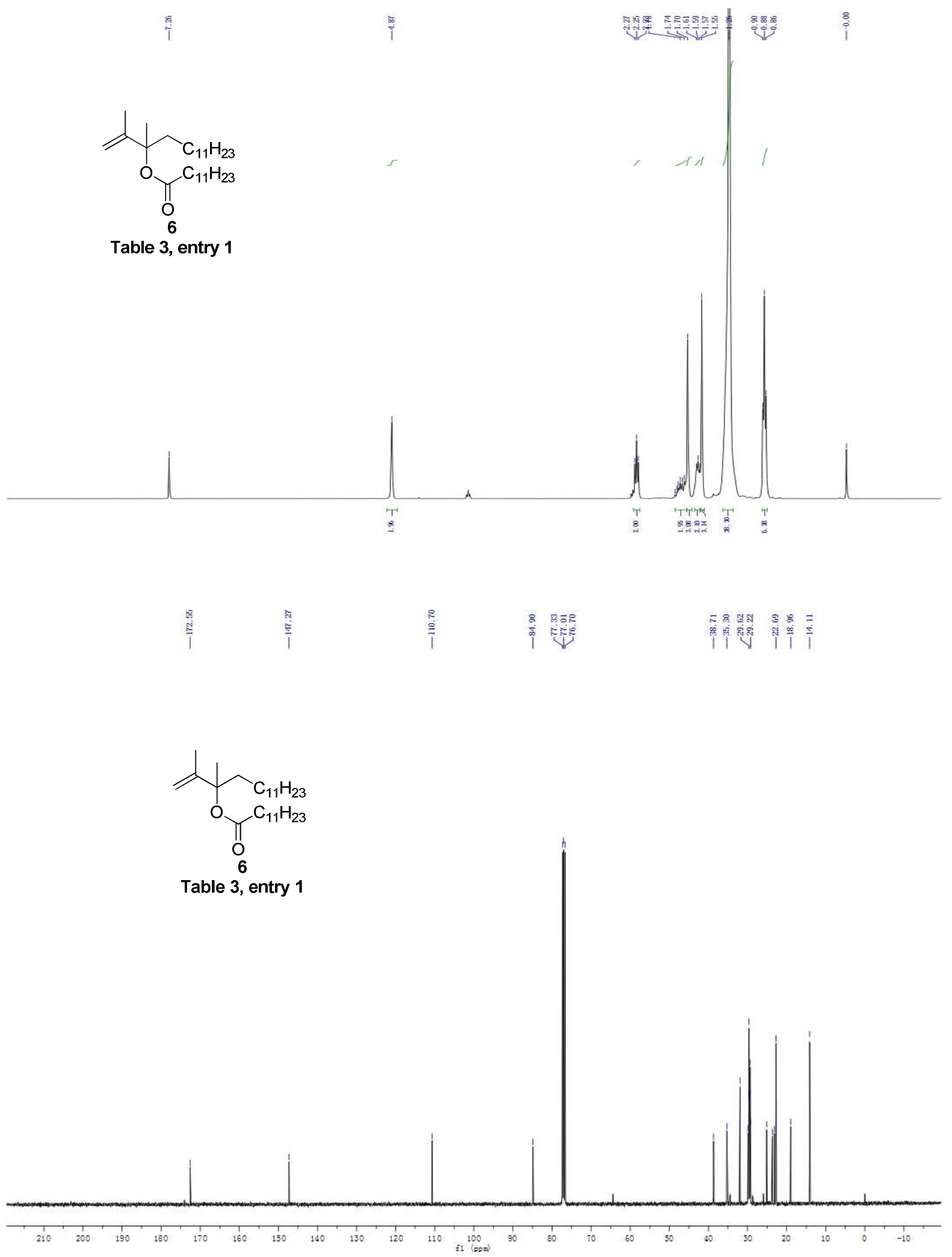


$\stackrel{2}{1}$<smiles>C=C(C)C(C)(CCC(Br)(Br)Br)OC(=O)CC(Br)(Br)Br</smiles>

Table 3, entry 2
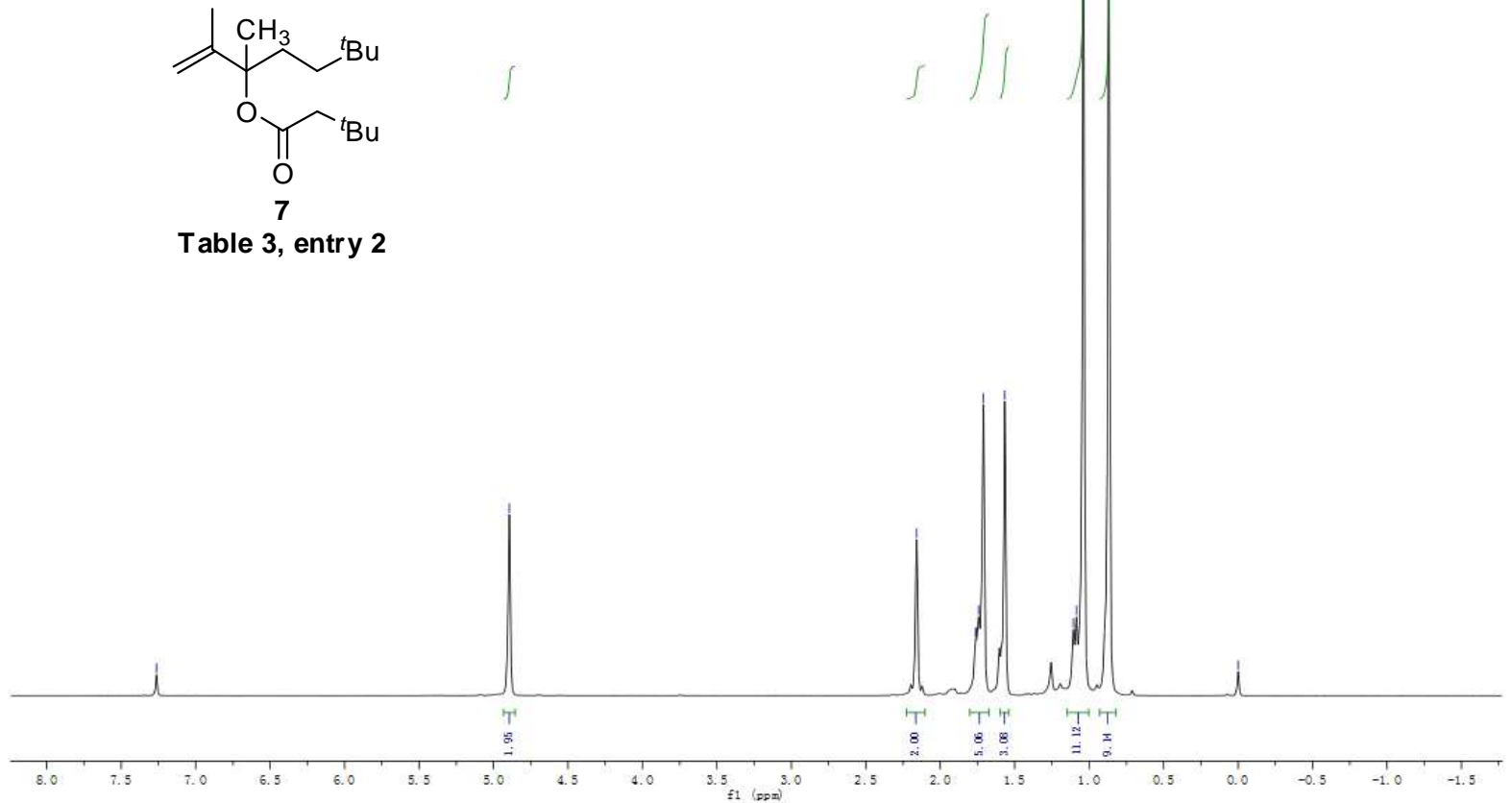

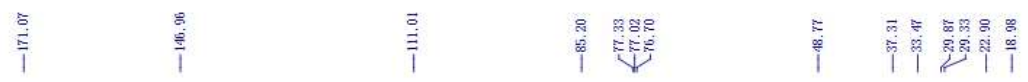

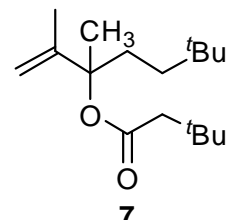

Table 3, entry 2

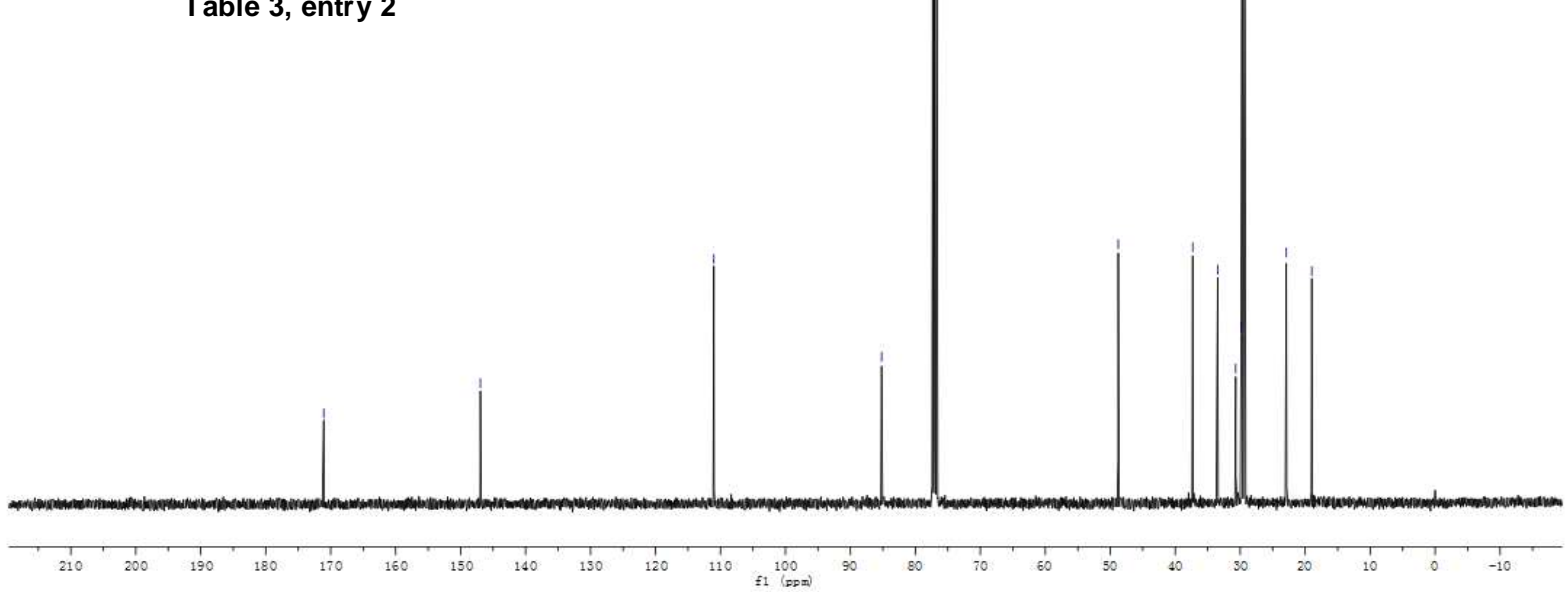




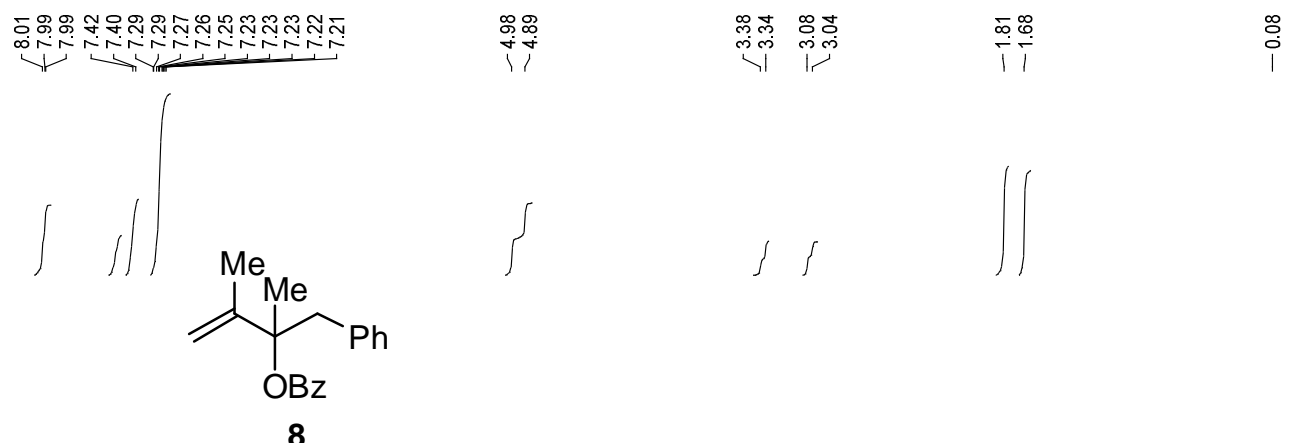

Table 3, entry 3

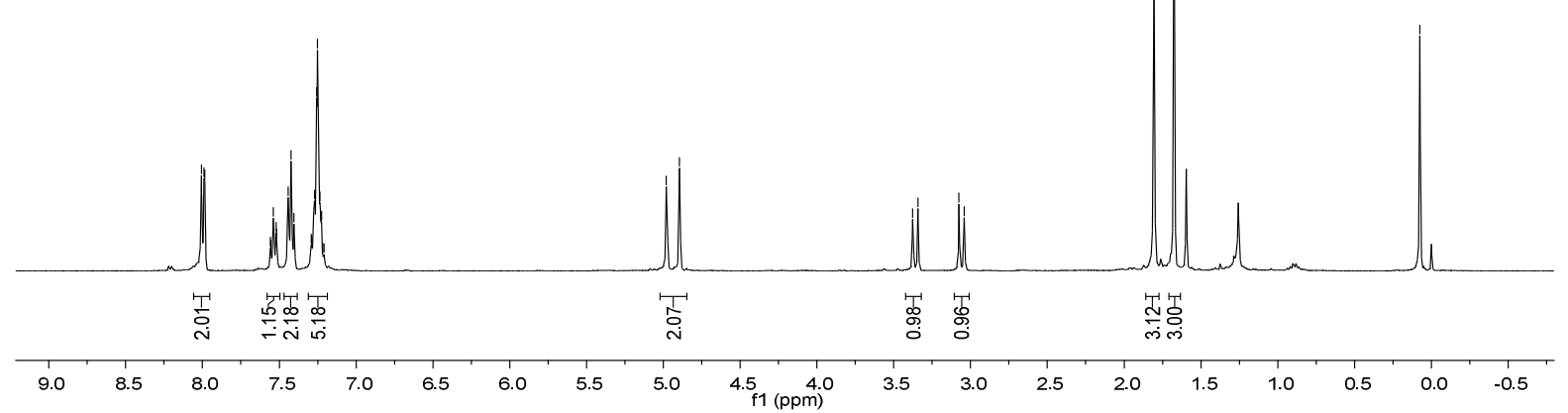




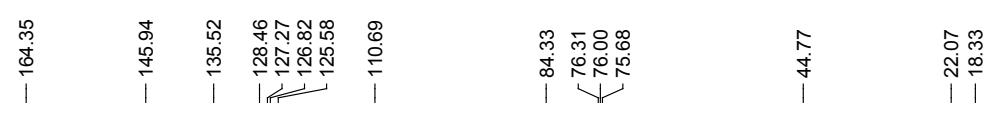<smiles>C=C(C)C(C)(Cc1ccccc1)OC(=O)c1ccccc1</smiles>

8

Table 3, entry 3

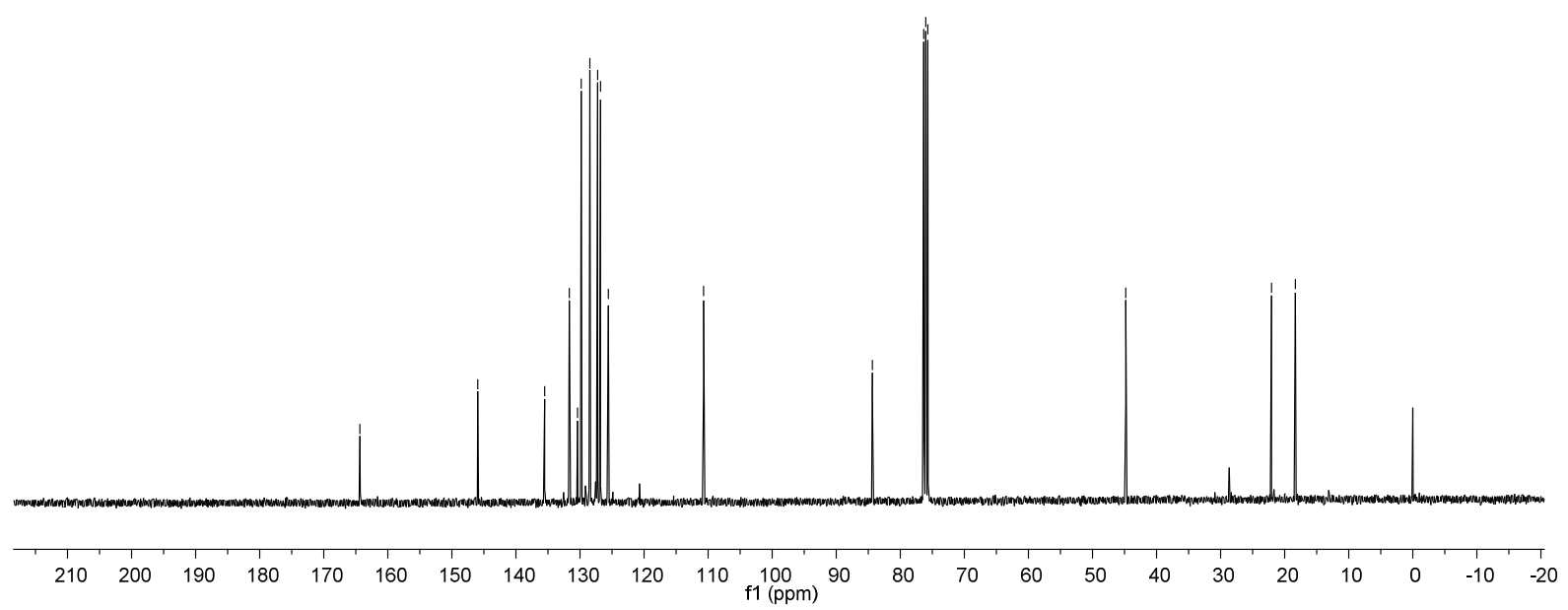

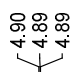<smiles>C=C(C)C(C)(CC1CC1)OC(=O)C1CC1</smiles>

9

Table 3, entry 4

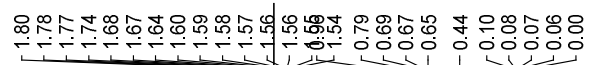

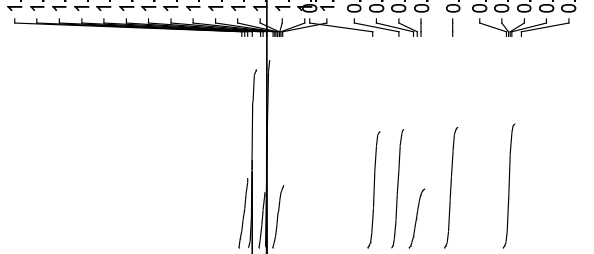

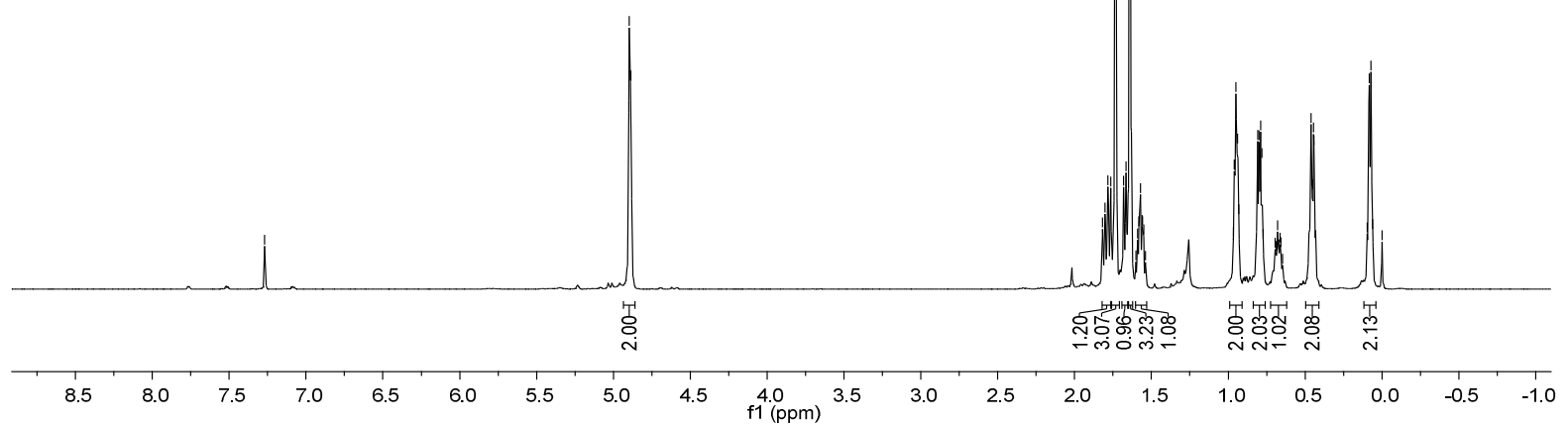




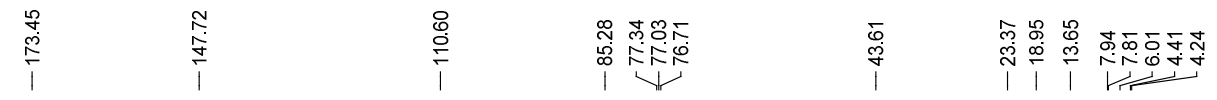

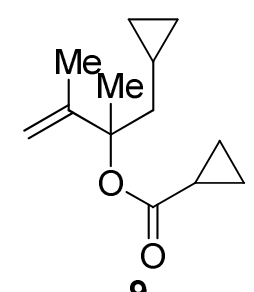

Table 3, entry 4

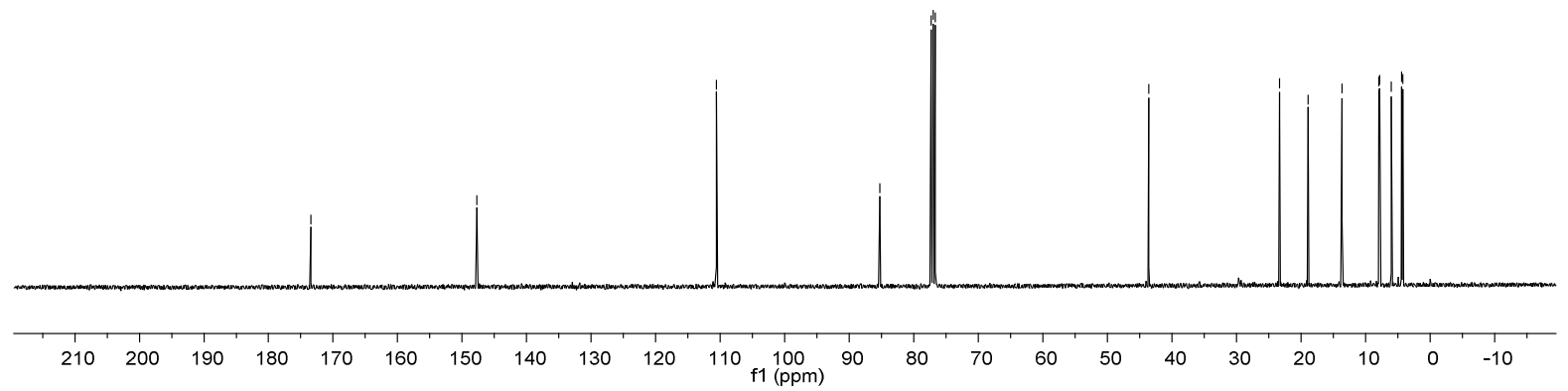

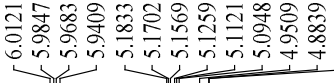

$\overbrace{\prod_{\mathrm{O}}}^{\mathrm{C}_{11} \mathrm{H}_{23}}+\underbrace{\mathrm{C}_{11} \mathrm{H}_{23}}_{\mathrm{O}_{\mathrm{O}}}+\overbrace{\mathrm{C}_{11} \mathrm{H}_{23}}^{\mathrm{C}_{11}}$

Table 3, entry 5

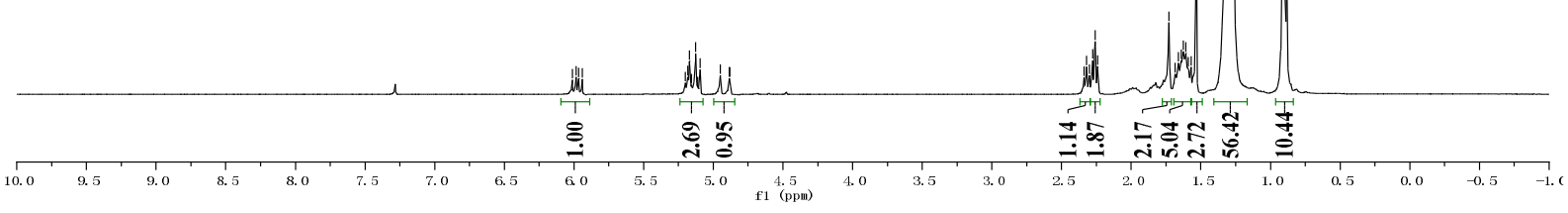




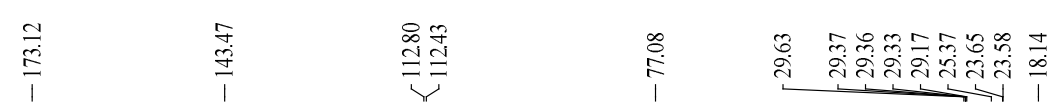

$\overbrace{\mathrm{O}}^{\mathrm{O}_{10}^{\mathrm{C}_{11} \mathrm{H}_{23}}}+\underbrace{\mathrm{C}_{11} \mathrm{H}_{23}}_{\prod_{\mathrm{O}}^{C_{11} \mathrm{H}_{23}}}$

Table 3, entry 5
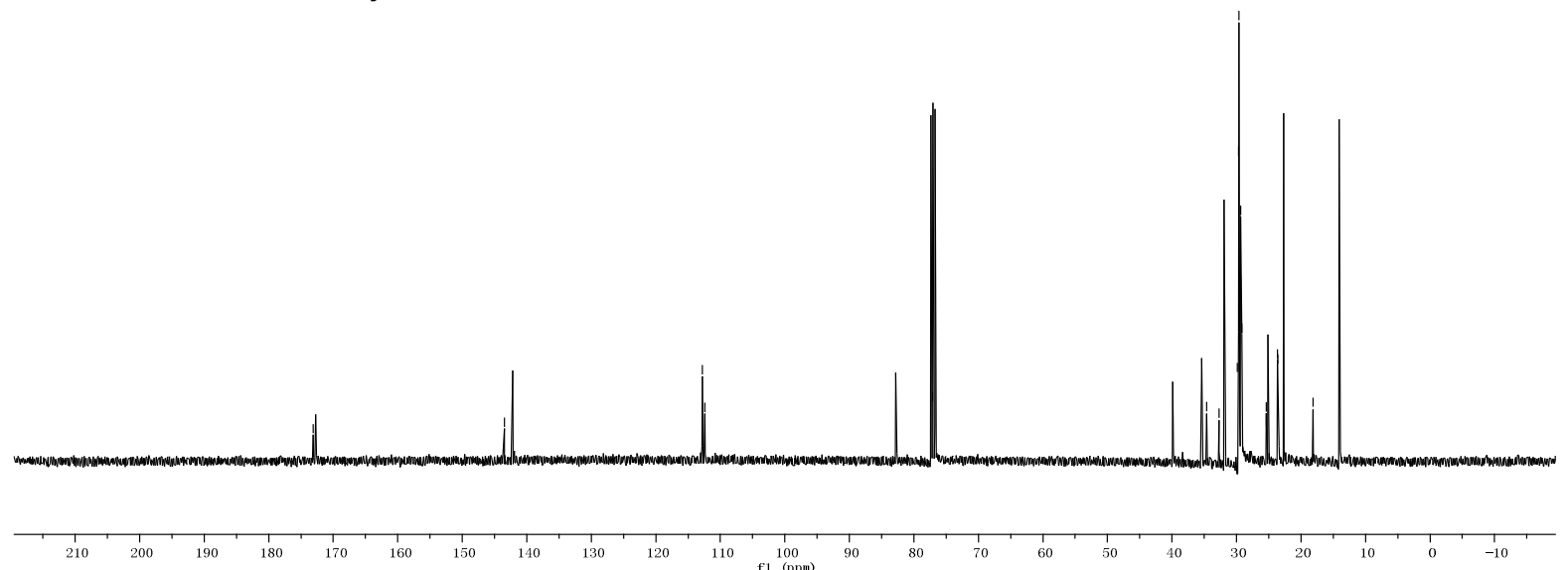

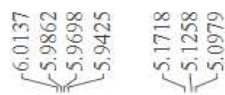

$\overbrace{\mathrm{O}}^{\mathrm{C}_{11} \mathrm{H}_{23}}$

10

Table 3, entry 5

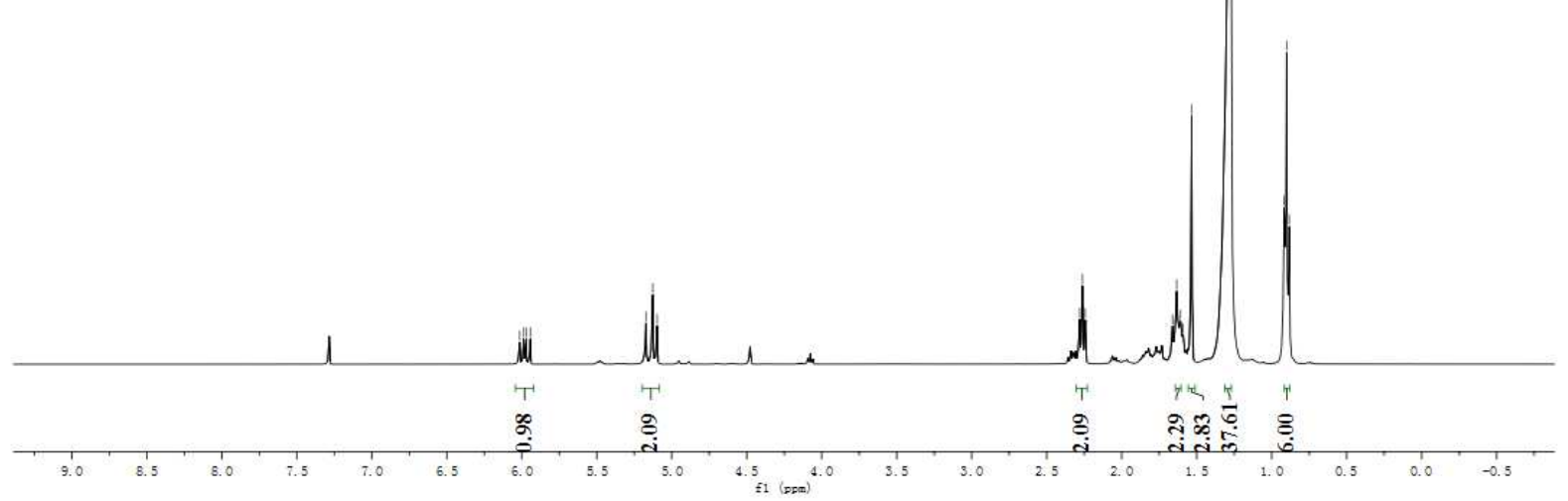




$$
\frac{1}{\frac{1}{2}}
$$

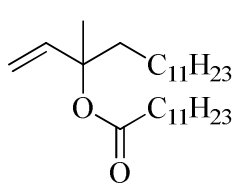

10

Table 3, entry 5

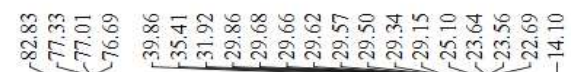
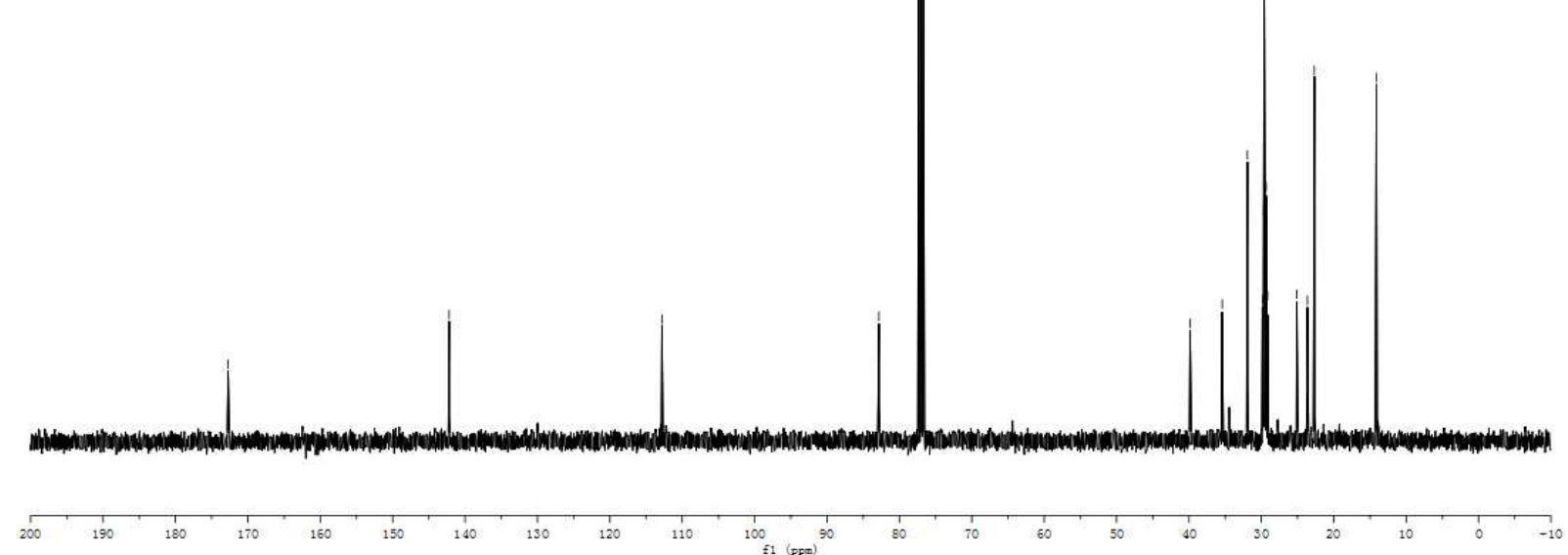

눈

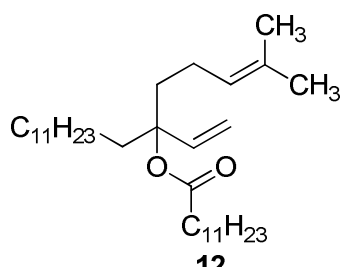

12

Table 3, entry 6

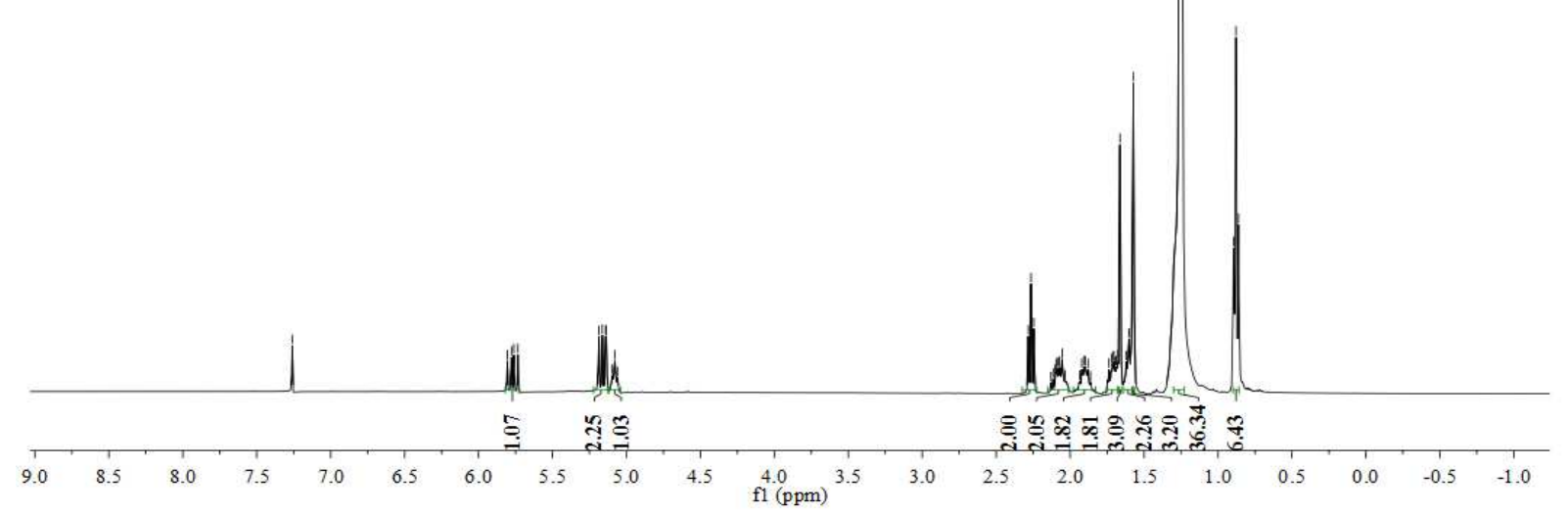




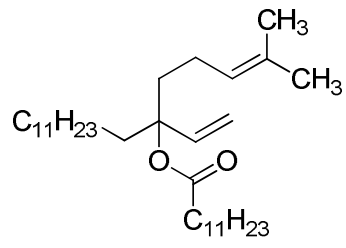

Table 3, entry 6

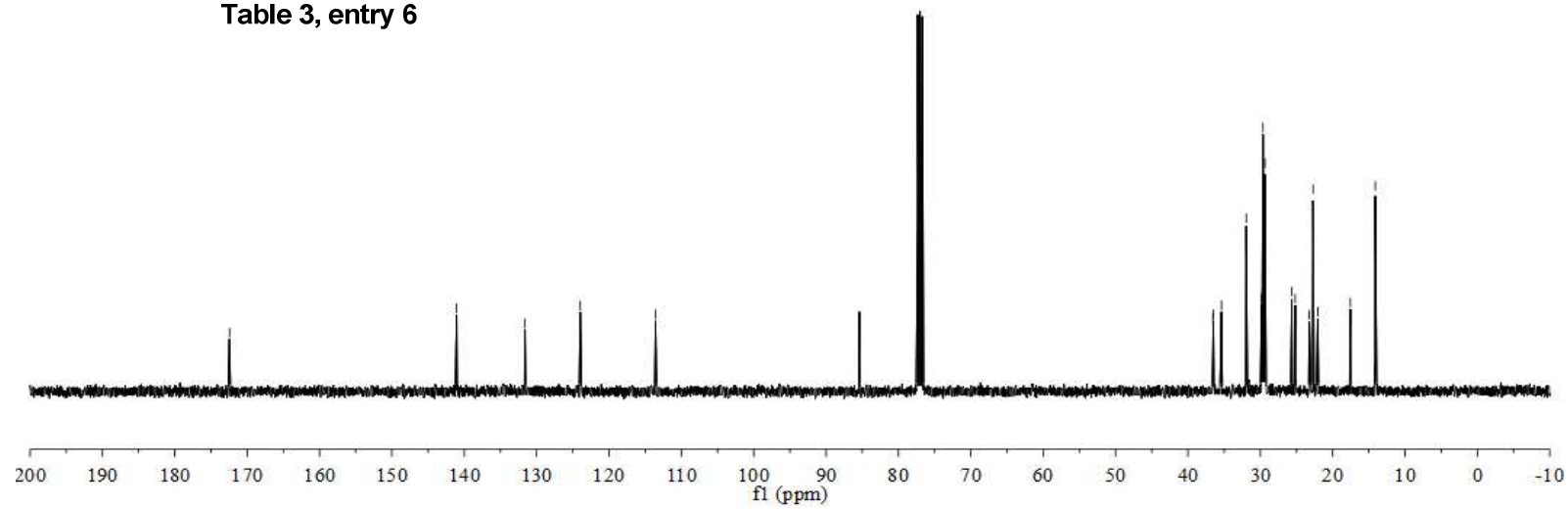

HSQC spectrum of product $\mathbf{1 2}$ :

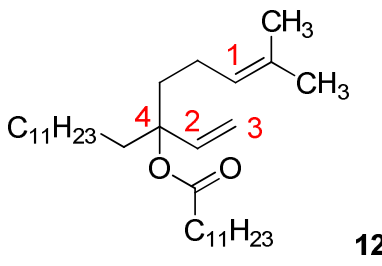




$$
1=4
$$




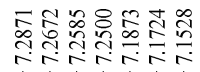

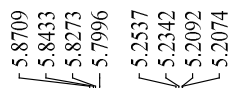

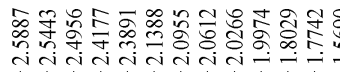

옹융

$\underbrace{\mathrm{C}_{2} \mathrm{H}_{4} \mathrm{Ph}^{t_{t}} \mathrm{Bu}}_{\prod_{13}^{t^{t}} \mathrm{Bu}}$

Table 3, entry 7

Table 3, entry 7

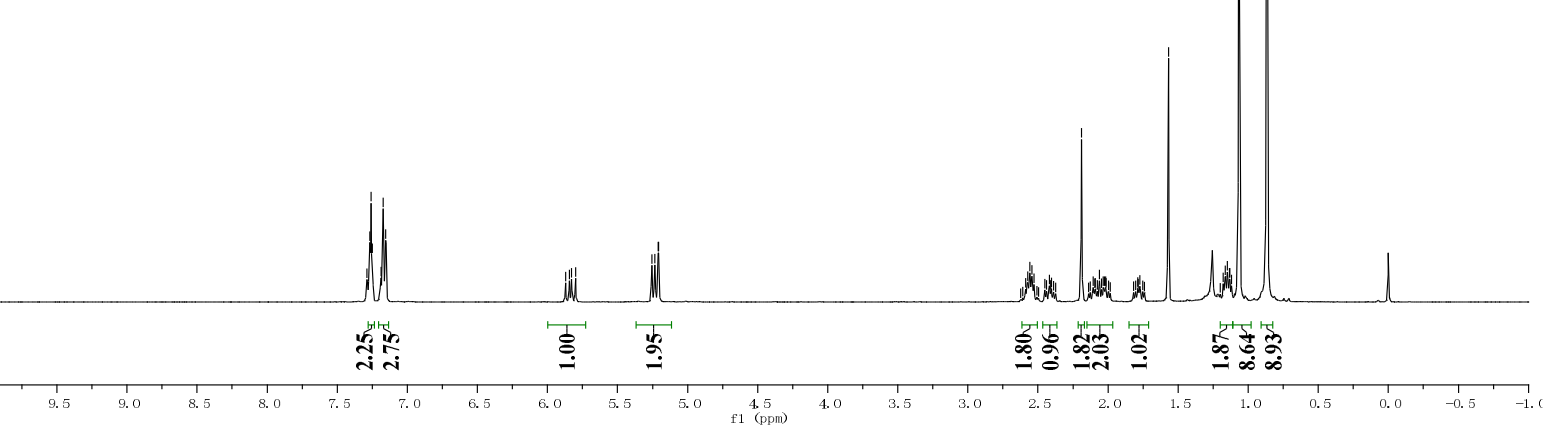

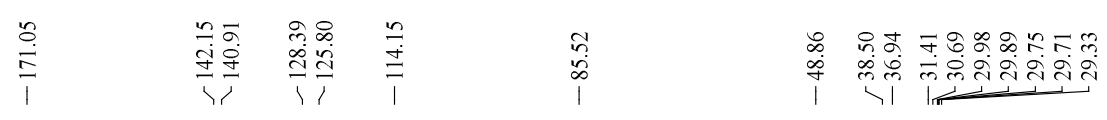

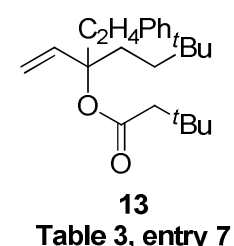

Table 3, entry 7
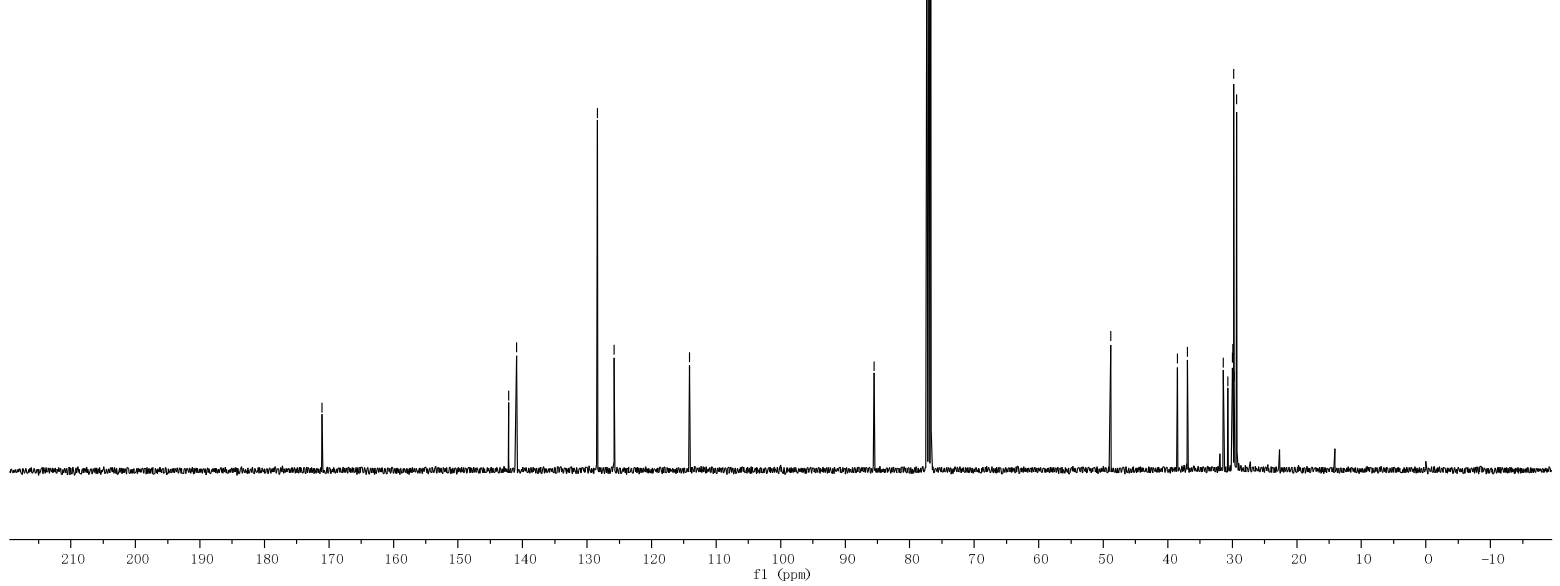


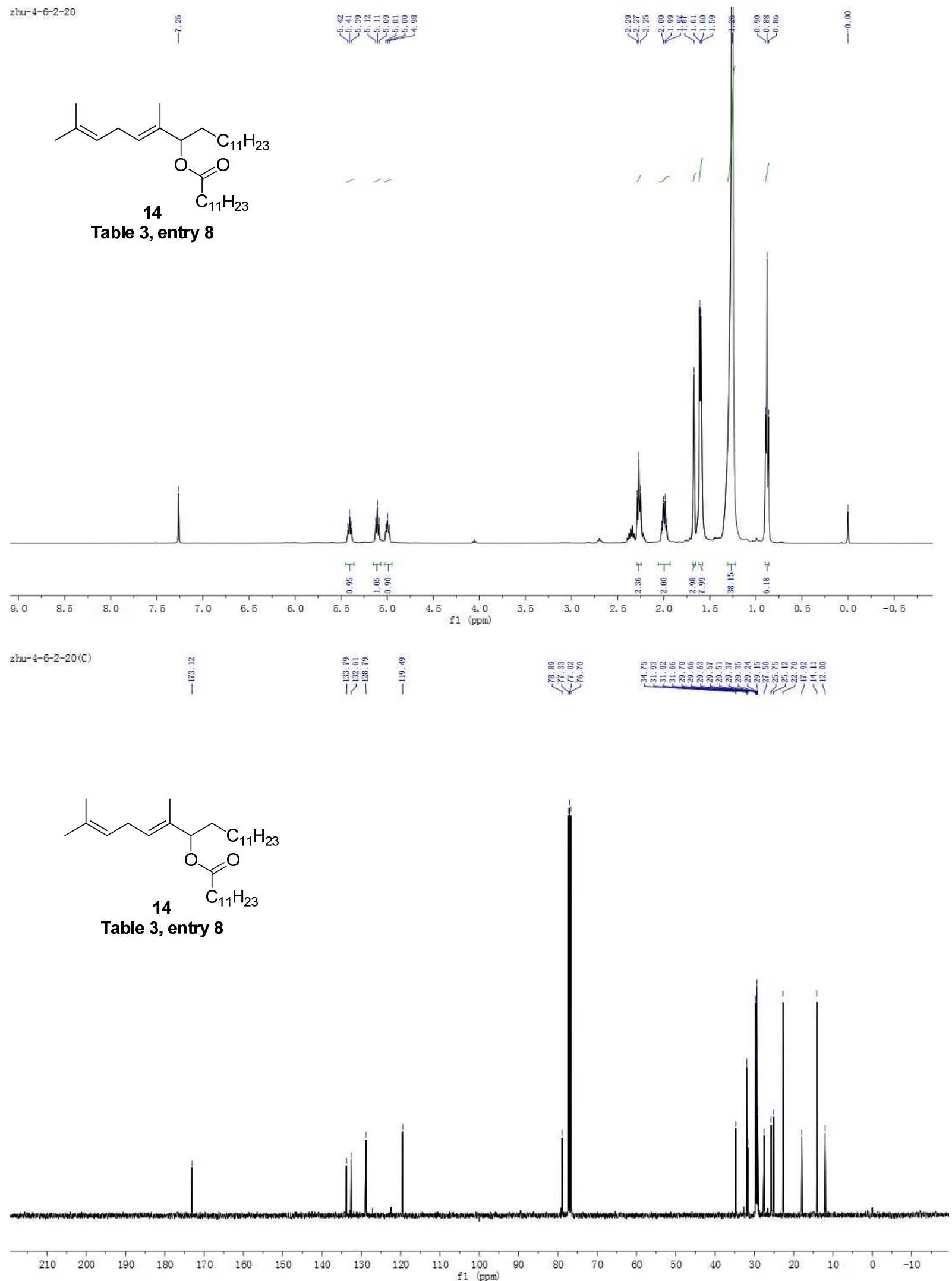




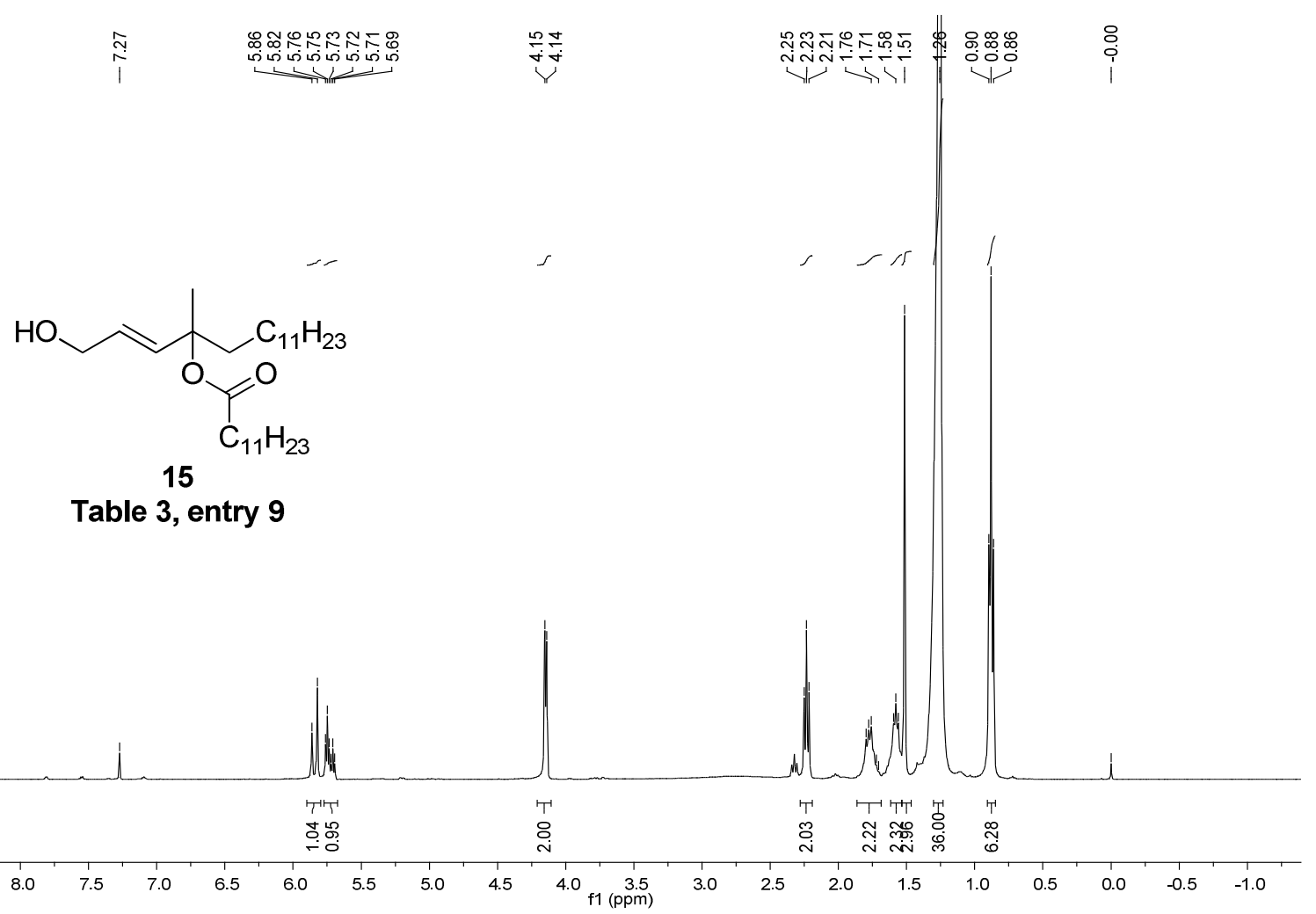

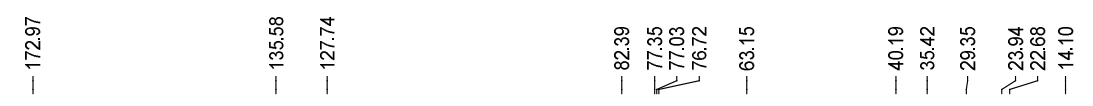

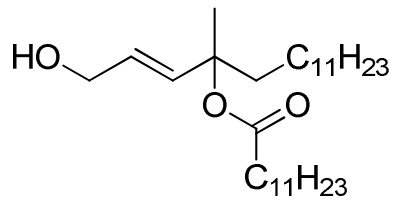

15

Table 3, entry 9

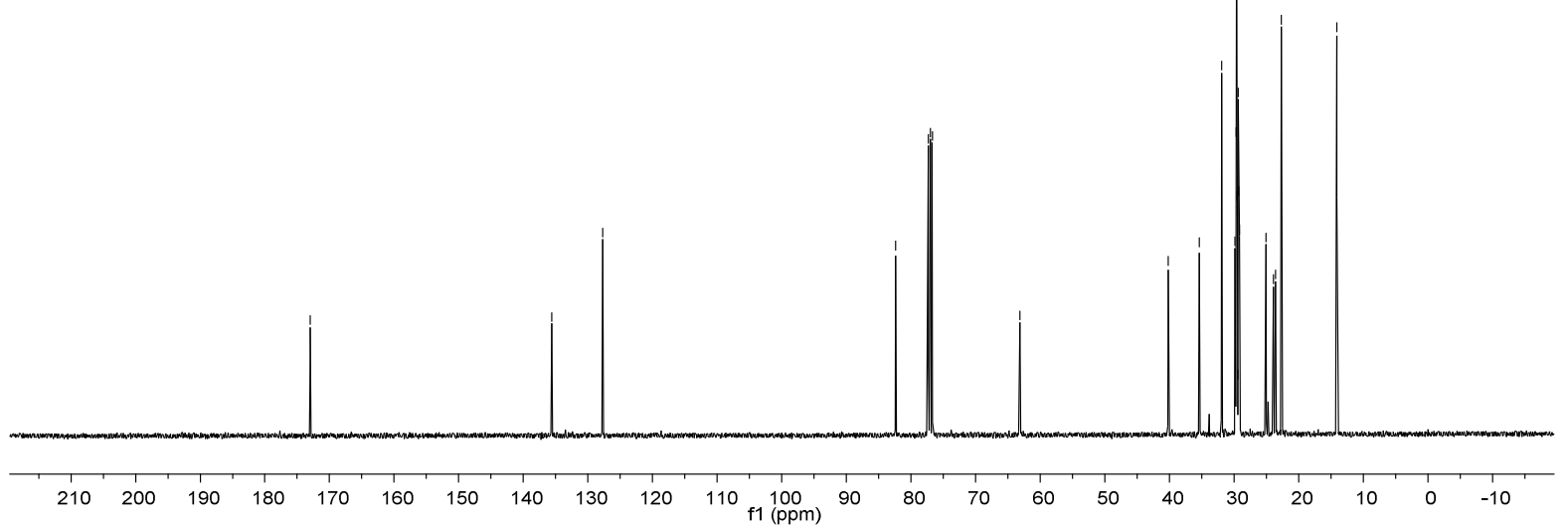




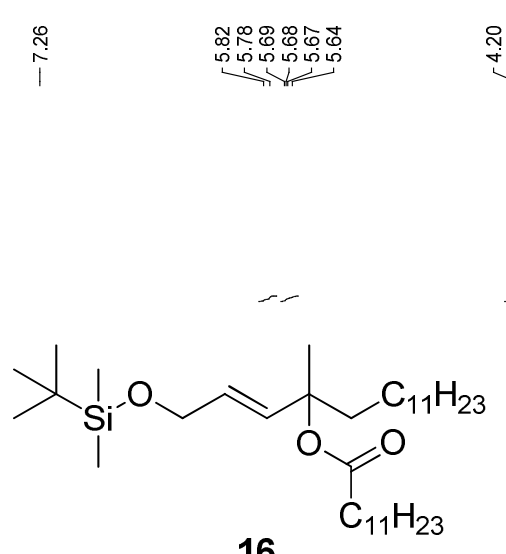

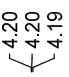

ن

Table 3, entry 10

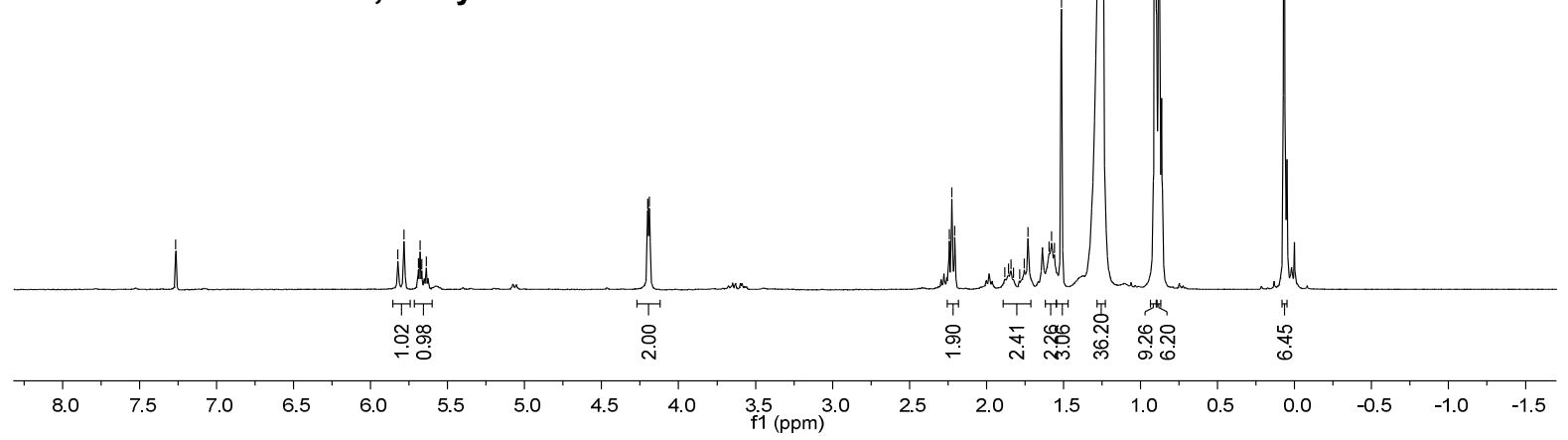

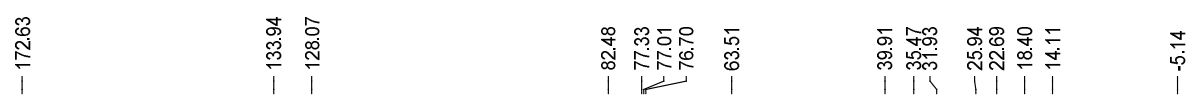

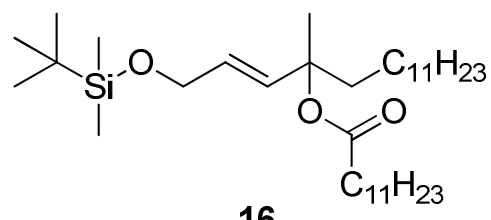

Table 3, entry 10

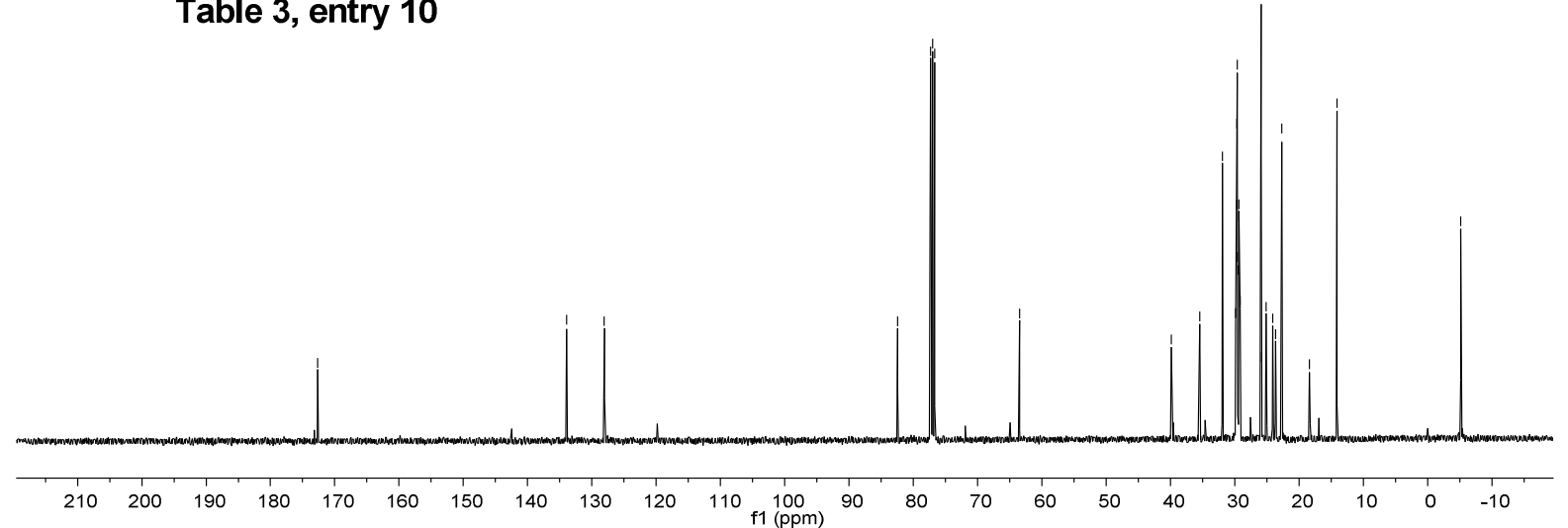




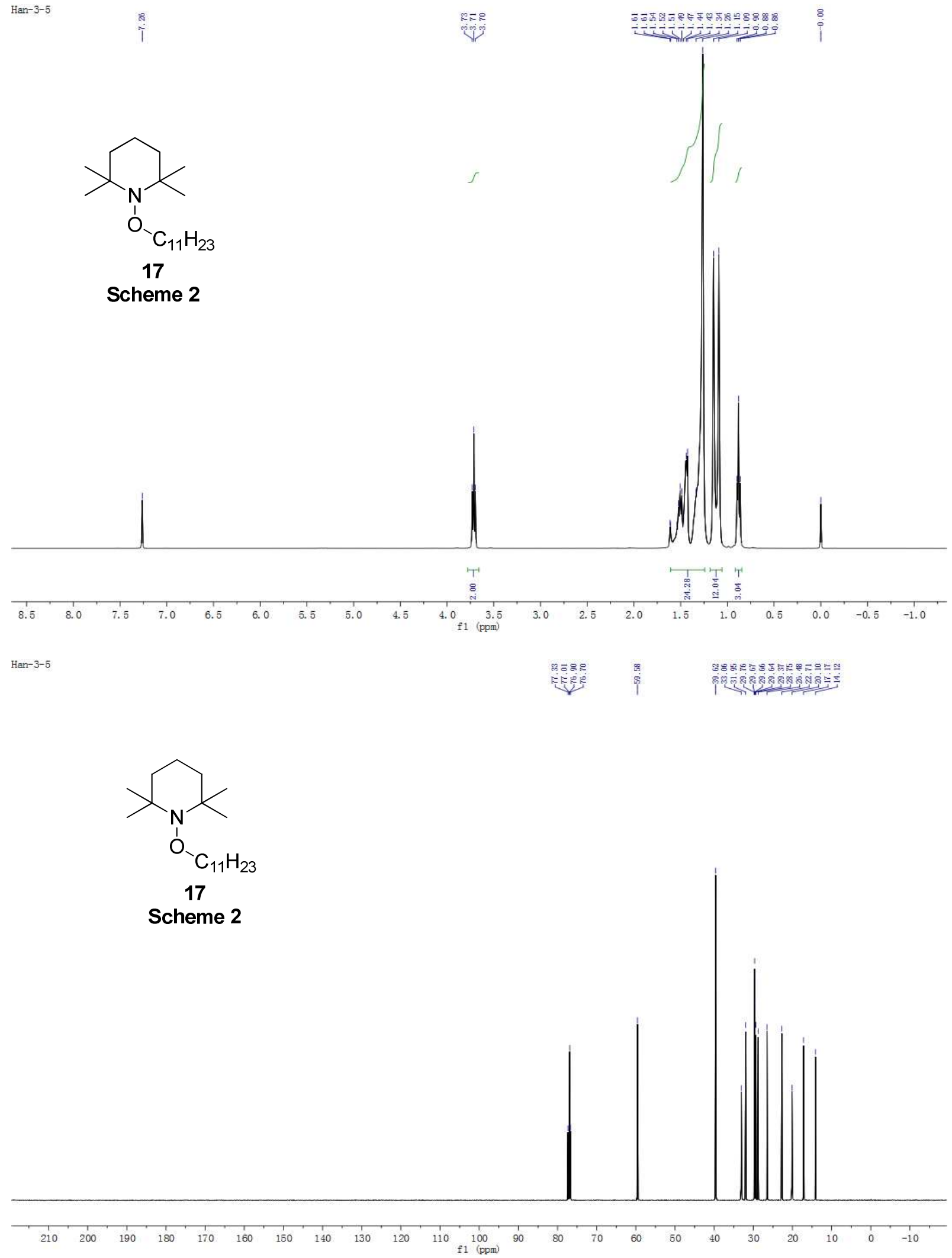




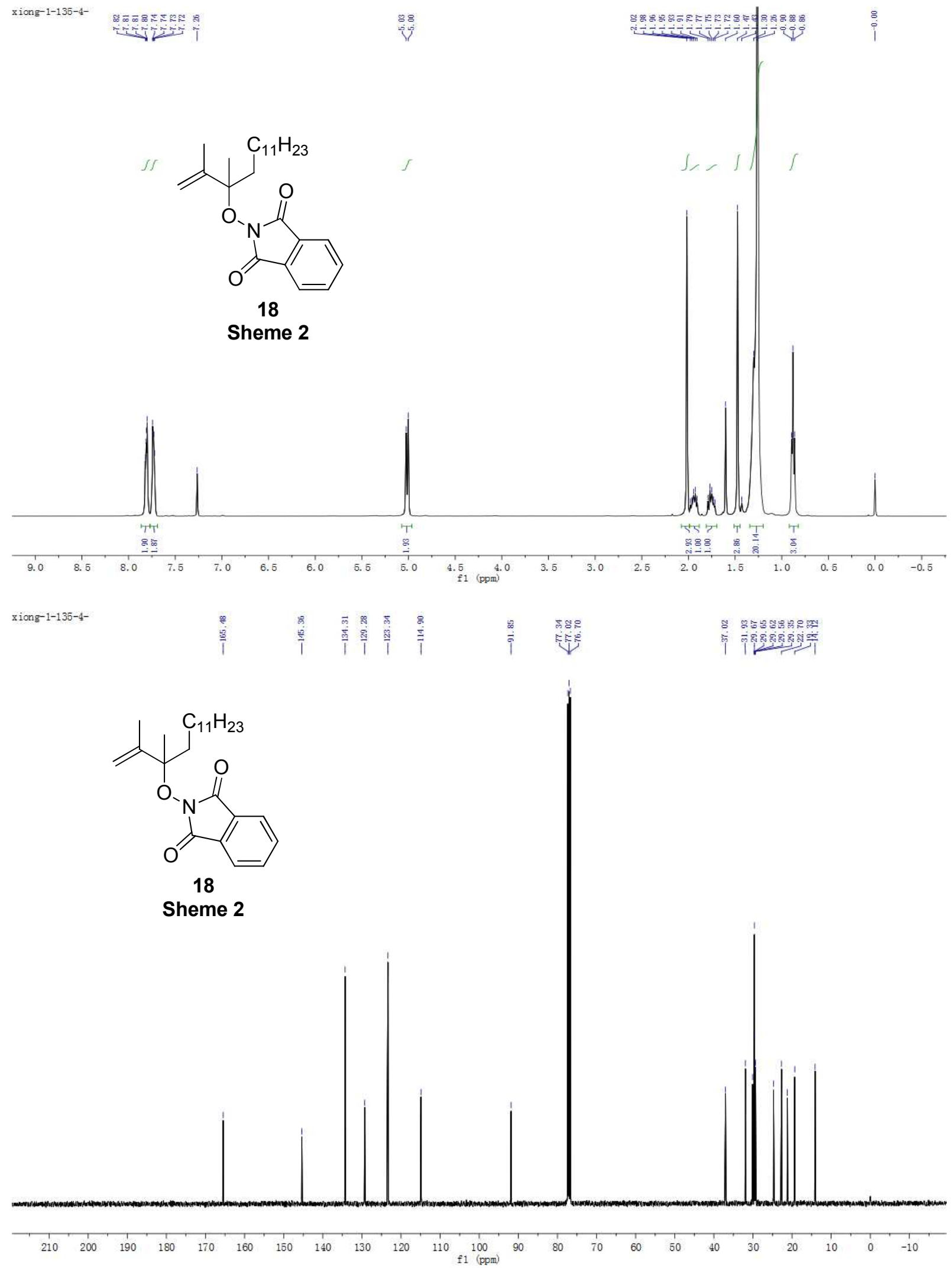


<smiles>C=CC</smiles><smiles>CCCCC</smiles>

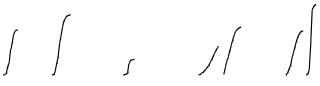<smiles>C=CC(CCCc1ccccc1)OC(=O)CCC(C=C)OC(=O)CCC(C)(C)C</smiles>

19

20

Scheme 3

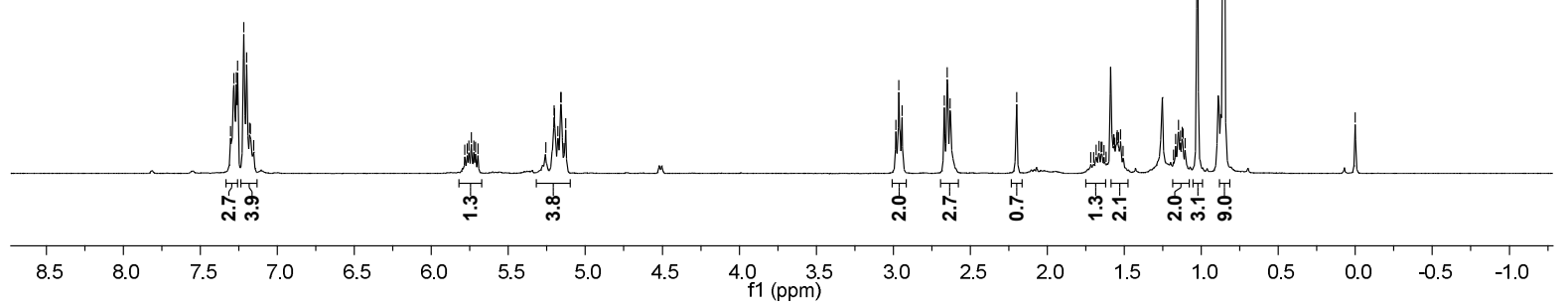

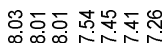

$\sqrt{\infty}$

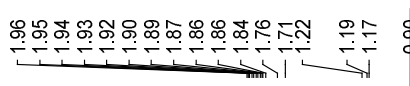

$\int \quad j$

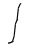

$\int \|$<smiles>C=C(C)C(C)(CCC(C)(C)C)OC(=O)OCc1ccccc1</smiles>

21

Scheme 3

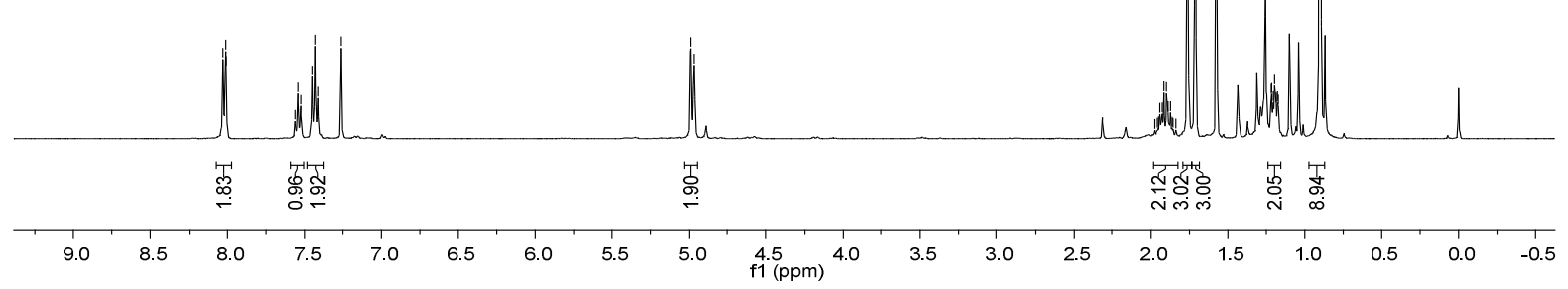




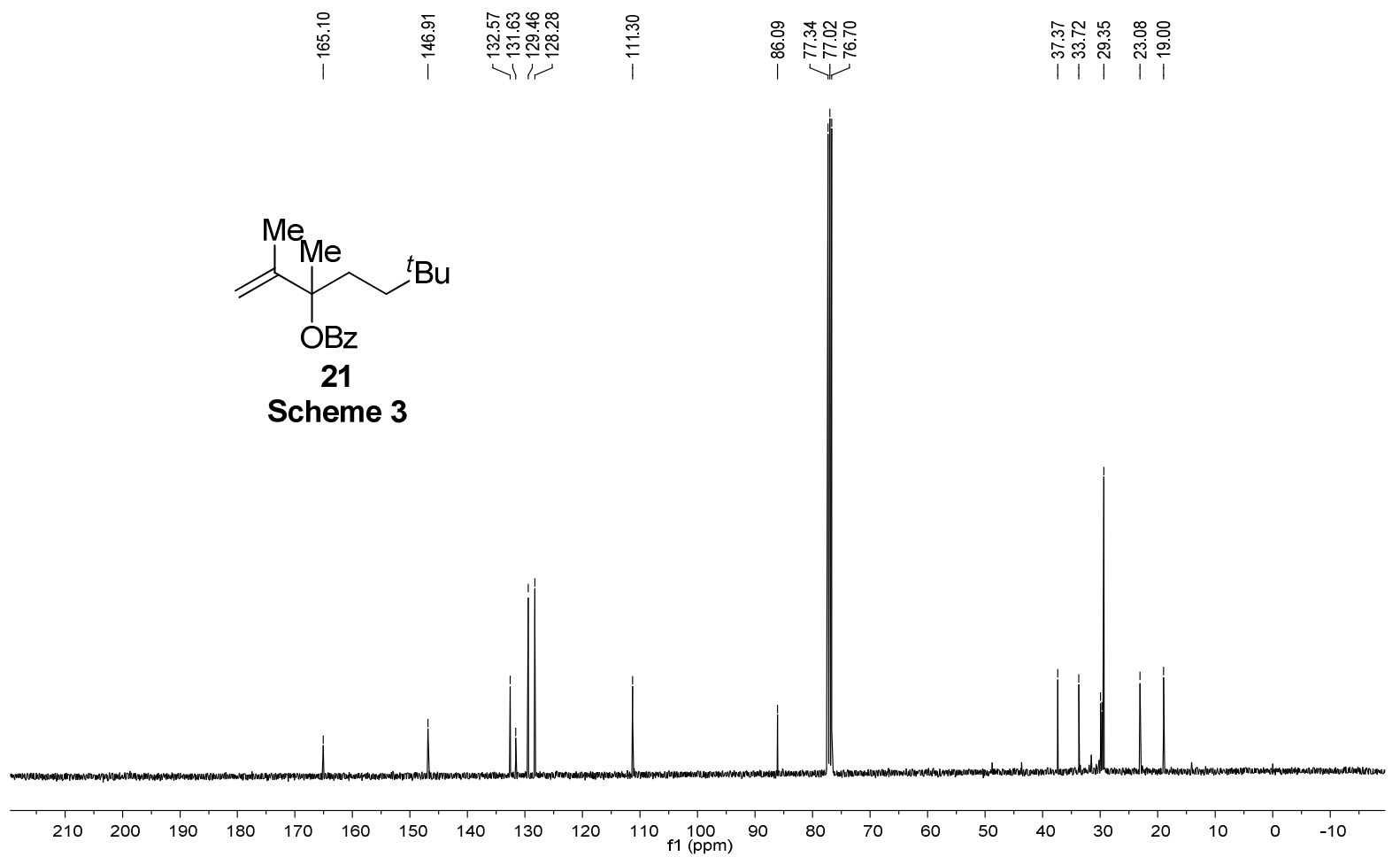

\section{References}

1. R. A. Sheldon, J. K. Kochi. J. Am. Chem. Soc.1970, 92, 4395.

2. M. Mori, K. Tonogaki, A. Kinoshita. Org. Synthesis. 2005, 81, 1.

3. A. P. Marcus, A. S. Lee, R. L. Davis, D. J. Tantillo, R. Sarpong. Angew. Chem. Int. Ed. 2008, 47, 6379.

4. M. T. Crimmins, R. S. Al-awar, I. M. Vallin, W. G. Hollis, Jr., R. O’Mahony, J. G. Lever, D. M. Bankaitis-Davis. J. Am. Chem. Soc. 1996, 118, 7513. 